Prepared for the U.S. Department of Energy under Contract DE-AC05-76RL01830

\title{
Simulating Interface Growth and Defect Generation in CZT - Simulation State of the Art and Known Gaps
}

CH Henager, Jr. (PI), F Gao, SY Hu, G Lin, EJ Bylaska (PNNL) Prof. N Zabaras (Cornell University)

November 2012

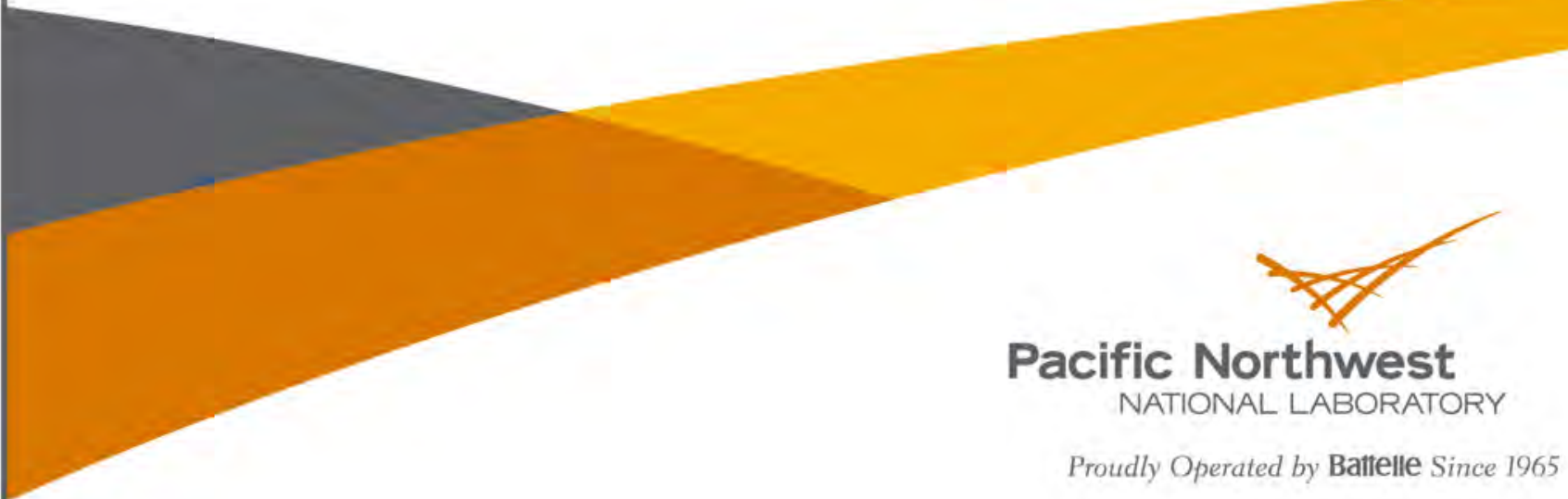




\title{
DISCLAIMER
}

This report was prepared as an account of work sponsored by an agency of the United States Government. Neither the United States Government nor any agency thereof, nor Battelle Memorial Institute, nor any of their employees, makes any warranty, express or implied, or assumes any legal liability or responsibility for the accuracy, completeness, or usefulness of any information, apparatus, product, or process disclosed, or represents that its use would not infringe privately owned rights. Reference herein to any specific commercial product, process, or service by trade name, trademark, manufacturer, or otherwise does not necessarily constitute or imply its endorsement, recommendation, or favoring by the United States Government or any agency thereof, or Battelle Memorial Institute. The views and opinions of authors expressed herein do not necessarily state or reflect those of the United States Government or any agency thereof.

\author{
PACIFIC NORTHWEST NATIONAL LABORATORY \\ operated by \\ BATTELLE \\ for the \\ UNITED STATES DEPARTMENT OF ENERGY \\ under Contract DE-AC05-76RL01830
}

Printed in the United States of America
Available to DOE and DOE contractors from the Office of Scientific and Technical Information,
P.O. Box 62, Oak Ridge, TN 37831-0062;
ph: (865) 576-8401
fax: $(865)$ 576-5728
email: reports@adonis.osti.gov

\begin{abstract}
Available to the public from the National Technical Information Service, U.S. Department of Commerce, 5285 Port Royal Rd., Springfield, VA 22161 ph: (800) 553-6847 fax: $(703) 605-6900$ email: orders@ntis.fedworld.gov online ordering: http://www.ntis.gov/ordering.htm
\end{abstract}

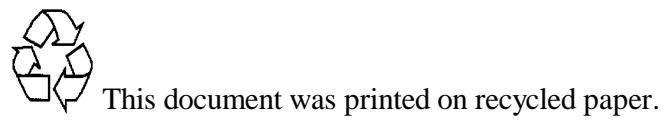


PNNL-22055

Simulating Interface Growth and Defect Generation in CZT - Simulation State of the Art and Known Gaps

CH Henager, Jr. (PI)

F Gao

SY Hu

G Lin

EJ Bylaska

Prof. N Zabaras

Cornell University, Ithaca, NY

October 2012

Final Report Prepared for

DNDO, TAR, Washington DC

Contract HSHQDC-11-X-00427

Pacific Northwest National Laboratory

Richland, WA 99352 
Table of Contents

1.0 Abstract/Outline of DNDO Modeling Approach ......................................................................... 1

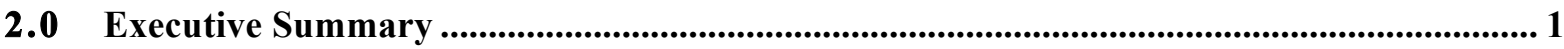

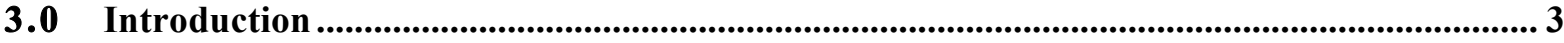

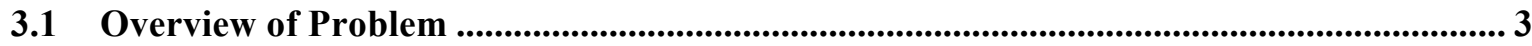

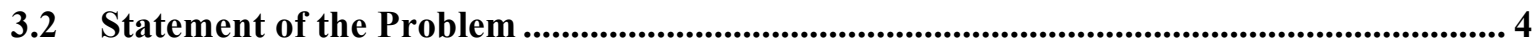

3.3 Outline of Approach to Address the Computational Modeling Problem ........................... 6

4.0 Assessment of the Current State-of-the-art for Crystal Growth Modeling - Methods and

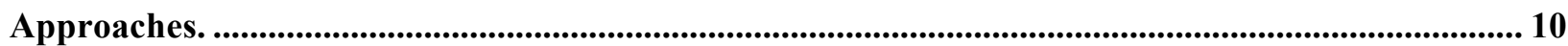

4.1 State-of-the-art Continuum Crystal Growth Models ........................................................... 10

4.1.1 Governing equations for continuum crystal growth models ......................................... 10

4.1.2 Methods for simulation of liquid-solid phase separation ............................................. 10

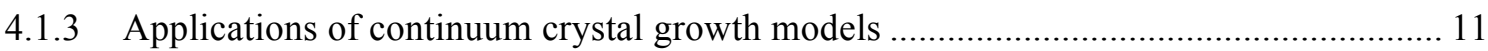

4.2 Fine-Scale Growth Models (Mesoscale Models) ..................................................................... 12

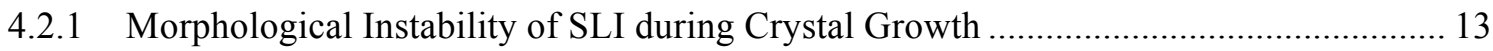

4.2.2 Defect Generation during Crystal Growth ............................................................ 16

4.2.3 Mesoscale methods of crystal growth and defect evolution .......................................... 19

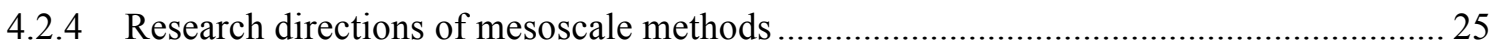

4.3 Atomistic Methods and Interatomic Potential Considerations...................................... 26

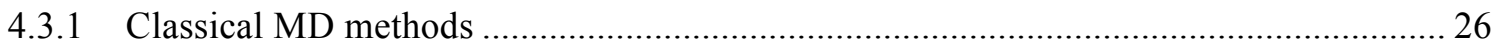

4.3.2 Embedded atom method and variants ..................................................................... 27

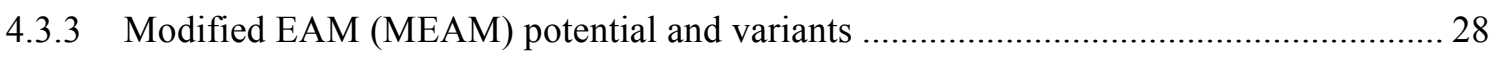

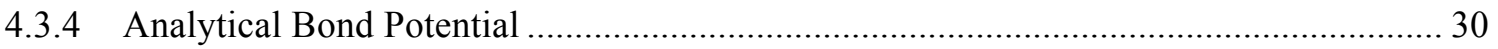

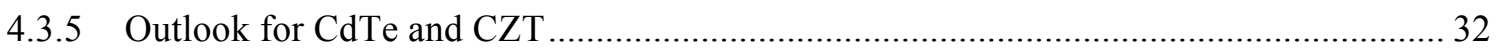

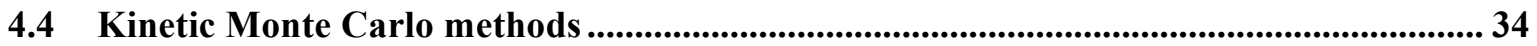

4.5 Ab Initio Methods and Approaches.......................................................................................... 38

4.5.1 Large Scale Electronic Structure Calculations................................................................ 38

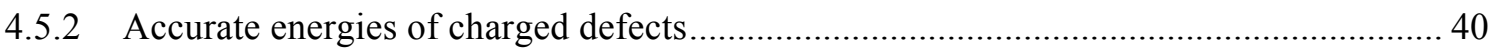

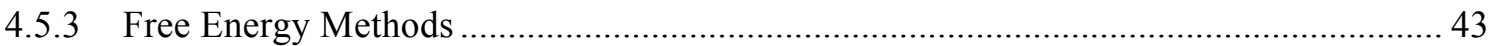

4.6 Uncertainty Quantification and Predictive Modeling ....................................................... 44

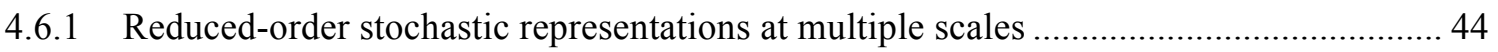

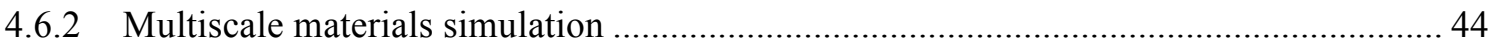

4.6.3 Means to systematically coarse grain while preserving essential statistics .................... 45

4.6.4 Ability to learn complex relations between processing, properties and structures .......... 46

4.7 Time-Scale Issues for Computational Performance .................................................... 47

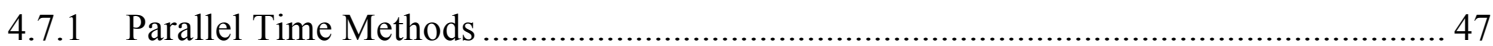

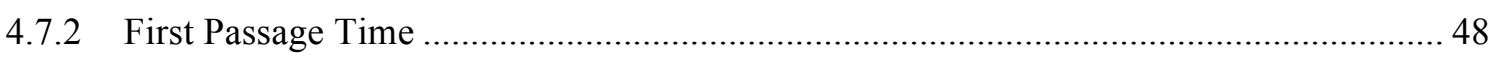

4.8 Semiconductor Response Function for Microstructural Effects on Gamma-Ray Absorption and Electron-Hole Transport .................................................................................... 48

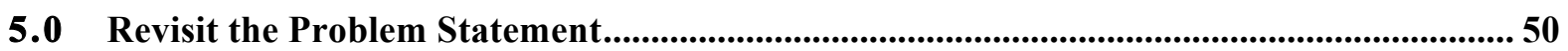

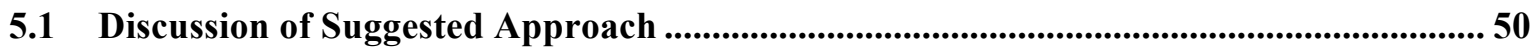


5.2 Identification of Knowledge or Method Gaps in Suggested Approach............................. 52

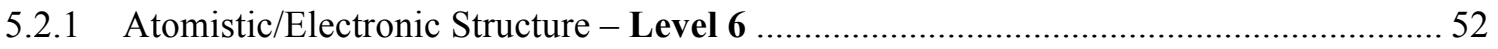

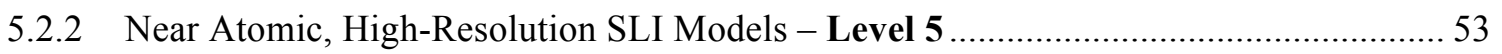

5.2.3 The SLI Growth Model with Concurrent Defect Generation - Level 4 .......................... 54

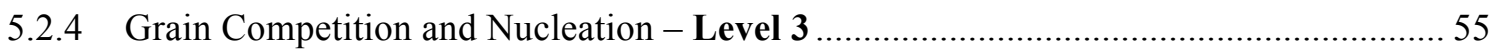

5.2.5 Global and Local Furnace Models - Levels 1 and 2 …................................................ 55

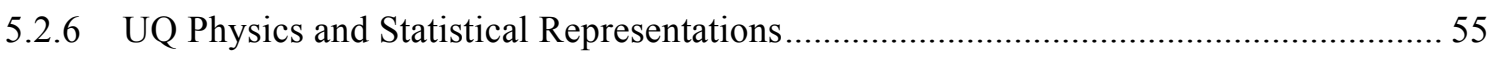

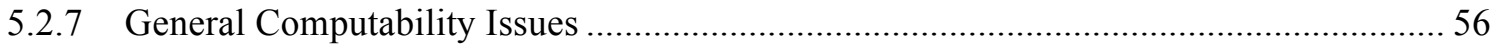

5.3 Suggestions for Closing the Gaps (Future Research Directions) .................................... 57

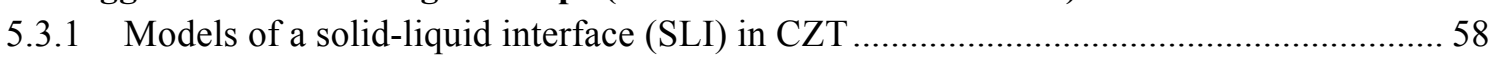

5.3.2 Research Direction 3: Semiconductor Response Models with As-Grown Defects ......... 59

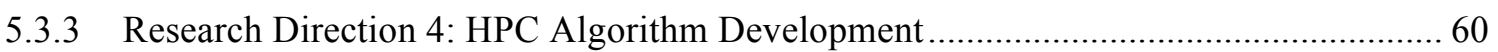

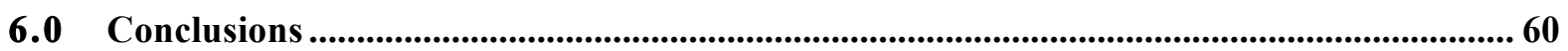

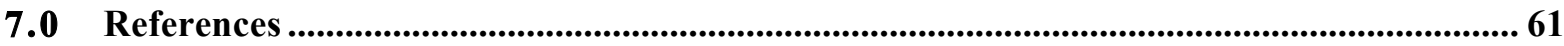




\subsection{Abstract/Outline of DNDO Modeling Approach}

A one-year Study Topic project was funded at PNNL to investigate the state-of-the-art in modeling CdZnTe (CZT) melt-solid interface, or solid-liquid interface (SLI), with an emphasis on knowledge and method gaps. The Study Topic culminates with this report to DNDO regarding the current modeling capabilities and outlining the known gaps in our capabilities. The report reviews the status of this area and discusses gaps and future research directions that can be used to provide resources to close these gaps in a gradual manner. At the outset it is recognized that the gaps include the well known multiscaling gaps that everyone encounters in these arenas, namely the coarse-graining problem having to do with time and length scale bridges from fine-scale atomistic data to coarse-scale mesoscopic models. There is no way around some of these gaps at present. However, by restricting the crystal growth models to something more focused and relevant to CZT growth we have identified four Research Directions to be explored and make recommendations for going forward with them. As a reminder, the scope of the Study Topic is included here:

This one-year, study topic project will survey and investigate the known state-of-the-art of modeling and simulation methods suitable for performing fine-scale, fully 3-D modeling, of the growth of CZT crystals at the melt-solid interface, and correlating physical growth and post-growth conditions with generation and incorporation of defects into the solid CZT crystal. In the course of this study, this project will also identify the critical gaps in our knowledge of modeling and simulation techniques in terms of what would be needed to be developed in order to perform accurate physical simulations of defect generation in melt-grown CZT. The transformational nature of this study will be, for the first time, an investigation of modeling and simulation methods for describing microstructural evolution during crystal growth and the identification of the critical gaps in our knowledge of such methods, which is recognized as having tremendous scientific impacts for future model developments in a wide variety of materials science areas.

\subsection{Executive Summary}

A comprehensive review of the current state-of-the-art in modeling SLI stability during crystal growth and in modeling defect evolution during crystal growth has been accomplished and used to reveal a series of gaps in our current modeling capabilities in applying such models to crystal growth of CZT. Thirteen of the gaps are in general model development issues and in crystal growth physics. Three of the gaps are in the relatively new area of uncertainty quantification, or UQ, that can be used to guide both model development and data sampling efforts for this daunting task. Two of the gaps are in computational areas having to do with increased computational efficiency for the anticipated methods.

Research Directions 1 and 2 are suggested for closing these gaps in a gradual manner for CZT, with the UQ gaps embedded within the attempts to develop crystal growth models, while the computational gaps are treated separately in Research Direction 4. A response model for gamma-ray energy resolution in defective CZT crystals is suggested as Research Direction 3.

It is recommended that a Research Direction 1 for crystal growth modeling focus on the stability during growth of a starting (111) seeded SLI for CZT. Solving this model will prove to be a substantial achievement and will relate, for the first time, furnace growth conditions to maintaining a stable (111) growth without competing cellular growth and new grain nucleation. This achievement will immediately suggest improved growth conditions for CZT since this is the preferred growth state and cannot always be maintained during VGF seeded growth. The utility of this approach as a first effort relies on the assumption that (111) stable growth would result in improved CZT materials since a flat growth interface 
in CZT has been experimentally linked to lower defect densities. This also allows research to proceed on an important topic with many of the same issues as a more comprehensive defect model by addressing a necessary stage of any CZT growth model, namely, what controls the SLI interface stability during growth?

Research Direction 2 is suggested to focus on the more difficult problem of simultaneously allowing SLI growth plus defect generation in the growing CZT solid. This is substantially more difficult due to uncertainties in nucleation rates of defects and unproven coarse-grain model development of highly coupled crystal growth events. For this model to be successful it must treat Te-particle genesis, dislocation generation, and point defect clustering during crystal growth. The main difficulty appears to be the long times required for the simulation and the large size of the highly-resolved models in order to capture dislocation cells formed during CZT cool down. This model, in order to treat the dislocation cell problem, is substantially larger than that required for Research Direction 1. Breaking up the effort into these two research directions allows substantial progress to be made without requiring the entire problem to be treated. Surely, any active research in this area would likely proceed in this staged manner.

Both Research Directions 1 and 2 require substantial work in determining defects in CdTe or CZT with a likely need for charge transfer using highly accurate quantum mechanics methods, such as DFT. Both require substantial understanding of energy landscape sampling and quantification along with a robust uncertainty quantification effort to guide the sampling and coarse-grain model development. It is appreciated that not everything from the finest resolution scales can be incorporated into a coarser resolution scale but currently this is a black art. This report suggests that this gap can be closed with some clever research.

It is also noted that there is a great need for Research Direction 3 to develop a validated CZT radiation detector response model to allow a target CZT defected microstructure to be computed. It is difficult to proceed along a path to minimize as-grown defects if there is not an understanding of the mechanisms for degradation in energy resolution in CZT for particular types of defects. A physically based CZT response model would help answer these questions. It is noted that current research in similar methods for scintillators strongly suggest that this can succeed immediately.

The computational gaps require closing in Research Direction 4 if anticipated modeling methods are to be extended with efficiency to the exascale-computing regime. The goal of this research is to speedup computations by orders of magnitude.

We recommend that initial resources be supplied to follow Research Directions 1 and 3 to have the greatest impact on the science and technology of crystal growth.

- Research Direction 1 will provide, for the first time, a direct connection between solidification of CZT or CdTe with furnace growth conditions using a simulated (111) seeded growth state. The model, if successful, will provide the conditions for attaining and maintaining stable growth in this state under prescribed vertical gradient furnace growth conditions and as a function of growth temperature gradient, melt concentration, liquid convection, and growth stresses.

- Research Direction 3 will provide a response function that will, for the first time, relate specific crystal defects typical of as-grown CZT to energy resolution degradation so that the correct understanding of defect mitigation is developed for Research Direction 2 in the future. Without this understanding it is difficult to know how to optimize CZT growth with 
any certainty. This research can be accomplished with more certainty that any of the other tasks, to be best of our knowledge.

- Research Direction 1 can likely be accomplished with a competent team of researchers over a three period with an estimated 3 FTEs per year. This would be an important milestone in CZT growth modeling, namely the ability to predict (111) interface stability as a function of furnace growth conditions. The success of this task would suggest that other growth orientations could also be studied in this manner, such as for unseeded CZT growth.

- Research Direction 3 can follow the apparently successful NNSA scintillator effort that has produced NWEGRIM. Note, however, that about 5 years with approximately 4 to 5 FTEs per year of funding was required to obtain current capability in this area. It is hoped that building on this effort will reduce the overall cost and increase the probability of success.

Research Direction 2 is more problematic since the tightly coupled issues of dislocation production and Te-particle genesis present a much more difficult type of problem to solve. At this time, we can only state that there are methods to tackle the individual issues but that a true multiphysics model like this has not yet been accomplished by anyone. This research hinges on knowing the correct physics as well as solving some of the coarse-graining issues that plague this type of multiscale modeling effort. This effort will require substantial resources and an outstanding team of researchers to accomplish and success here may require aspects of Research Direction 4 to be addressed. Research Direction 4 can take advantage of existing computational mathematics teams, such as those working on the Fast Math computational centers.

\subsection{Introduction}

\subsection{Overview of Problem}

The growth of CZT from the melt has reached a critical stage where the need to understand the evolution of the as-grown microstructure during crystal growth is paramount. CZT is a difficult material to grow from the melt. The liquid is viscous, has low thermal conductivity, high melting point (1365 K) and has high vapor pressures for $\mathrm{Cd}$ and Te at its melting point. The CZT solid has low thermal conductivity, low critical resolved shear stress (CRSS), low stacking fault energies, and exhibits retrograde Te solubility on cooling $[1,2]$. It is cubic but exhibits strong melt growth anisotropy with growth instabilities in the $<110>$ and $<112>$ directions [3]. The solid is extremely soft at elevated temperatures with a CRSS of approximately $0.2 \mathrm{MPa}$ at the CZT growth temperatures so that dislocations are almost unavoidable during growth $[1,2]$. Therefore, it is necessary to construct a model of the microstructural evolution during crystal growth in order to fully understand to coupling of observed microstructures to growth conditions. With the aid of a microstructural response model of gamma-ray interactions, such as that provided by NWEGRIM [4-6], this type of approach would allow growth optimization of CZT to achieve improved gamma-ray energy resolution.

The status of modeling the growth of CZT from the melt is complex as there are many types of models to consider. For this report we will restrict the type of growth to vertical Bridgman growth, also termed vertical gradient freeze (VGF) or low-pressure Bridgman growth, although these modeling methods can be applied to other common growth geometries, such as traveling heater methods (THM) with little changes. At the level of the growth furnace and the melt crucible and treating the solid and liquid as continuum objects with specified physical and mechanical properties there are several key references regarding advances made in models and modeling approaches [7-10]. The main technical 
achievement in these models is the ability to accurately model the physics of CdTe and CZT in terms of heat transfer, including optical transparency, and to be able to couple two modeling scales in a selfconsistent manner. These models can include magnetic fields [11-15] and crucible rotations [16-22] and can be used to guide the furnace growth parameters to obtain a preferred flat or convex ${ }^{1}$ growth interface for CdTe or CZT [23]. The use of continuum models has led to improved growth of CZT or CdTe crystals but the level of defects in even the best CZT crystals is too high, on average, to allow the routine production of high-resolution detectors [24-28] with theoretical energy resolution.

It is not enough to model the shape of the CZT or CdTe interface coupled to the furnace growth conditions because local solid-liquid interface instabilities are complex and can arise due to either thermal fluctuations, fluid flow in the melt, or compositional fluctuations at the solid-liquid interface [1, 2, 29]. In addition, growth and deformation twins can form together with a dislocation network due to thermal growth strains such that a continuum model cannot capture enough detail to couple these events to the furnace growth parameters. Therefore, a more detailed or fine-scale approach is required to capture these details, typically at the length scale of the various microstructural features, the so-called mesoscale. At this scale the microstructure of the growing crystal is resolved and related to the growth parameters set by the furnace growth conditions. This is an area of keen interest today, as typified by the recent DOE Workshop Report ${ }^{2}$ that discusses the challenges and opportunities in mesoscale science.

There is little doubt at present that crystal defects in CZT or CdTe control the response of the material in terms of energy resolution and other important radiation operational parameters [24, 30-39]. These defects, including twins (deformation and growth), growth facets or growth fingers, dislocations and dislocation subgrains, and Te-particles, form during crystal growth as a result of the crystallization process that is controlled by the furnace growth conditions and by the type of crucible used to contain the CZT or CdTe melt [40]. If there is to be progress in understanding the nature of CZT growth and optimization of the growth then modeling must be able to capture and relate the formation of each of these defects to the furnace growth conditions. This is the challenge of these fine-scale growth models.

The goal of this study was to determine how to model the crystal growth process at the solid-liquid interface (SLI) in such a way that crystal defects could be incorporated directly into the growth model. This requires a much different approach than the continuum models, but any discrete or fine-scale modeling effort would also have to be consistent with such continuum models so that the proper heat transfer and growth rates were maintained.

\subsection{Statement of the Problem}

Models of the "growth of CZT crystals at the melt-solid interface (or SLI), and correlating physical growth and post-growth conditions with generation and incorporation of defects into the solid CZT crystal $^{3 "}$ " must necessarily consider the following outcomes or realizations as output from a hypothetical model:

1. The model needs to consider solidification at the SLI where the solid fraction in the model increases with time and the liquid fraction decreases with time, i.e., the interface moves.

\footnotetext{
${ }^{1}$ The term convex here refers to the shape of the solidified portion of the growth interface with respect to the solid.

2 "From Quanta to the Continuum: Opportunities For Mesoscale Science", a Report from the Basic Energy Sciences Advisory Committee, Chair: John Hemminger University of California, Irvine, U.S. Department of Energy September 2012, Prepared by the BESAC Subcommittee on Mesoscale Science.

${ }^{3}$ This sentence from the Statement of Work for this contract succinctly captures the goal of any proposed modeling approach but requires added detail.
} 
2. The model needs to track or include details in the liquid ahead of the solidification front so that concentration, thermal, velocity or advection, and liquid structural gradients are tracked.

3. The model needs to be large enough and detailed enough to capture:

a. SLI dynamics, which implies a length scale from nanometers to 100's of microns.

b. Te-particle genesis routes via hypothetical mechanisms including nucleation and growth, precipitation, direct capture from the melt, and ingress via dislocation core diffusion or planar defect diffusion pathways.

c. Dislocation subgrain networks and twin formation mechanisms, which implies a length scale from nanometers to several hundreds of microns.

d. Point defect formation, migration, and clustering.

4. The model must be able to compute to times on the order of diffusion over a typical microstructural feature, or time to develop a Te-particle facet, or time to form an equilibrium dislocation cell substructure, or time to allow hypothetical Rayleigh instability to develop for Te-particle genesis from dislocation cores.

Together, these constitute a serious computational challenge, as follows:

1. The model size should be on the order of $1-\mathrm{mm} \times 1-\mathrm{mm} \times 5-\mathrm{mm}$ in extent with the long dimension normal to the solid-liquid interface since dislocation cell sizes in CdTe are on the order of $200 \mu \mathrm{m}$ in diameter.

2. The model should be fully $3 \mathrm{D}$ and could consist, as a discrete model for example, of $1-\mu \mathrm{m}$ cubical volume elements so that $5 \times 10^{9}$ volume elements are treated with suitable resolution of gradients, morphologies, and fields. This puts the Te-particle resolution at about 1- $\mu \mathrm{m}$, which matches optical microscopy limits. A model of this size contains $\sim 2.95 \times 10^{19}$ atoms.

3. The model should allow the solid-liquid interface to progress at least 1-mm during the simulation, which requires $2 \mathrm{~h}$ of real time given a typical VGF growth rate of $0.5 \mathrm{~mm} / \mathrm{h}$.

4. The model should have a time resolution of $1-\mu$ s in order to capture defect diffusion, which will require about $1 \times 10^{10}$ time steps. This puts severe constraints on computational solution times. This suggests that solution times per time step are on the order of $10^{-4} \mathrm{~s}$ to achieve a reasonable computational time in real time.

Further, the model is considered to focus on the evolution of a "statistically probable as-grown microstructure" from the melt in a single oriented grain of CZT for a reasonable simplification since multiple grain interactions affect SLI morphological instability as well as defect generation, but these multiple grain interaction effects are not necessary for a model that treats crystal growth and concurrent defect generation. The model treats the liquid as a complex fluid with thermal, compositional, and velocity fields imposed by suitable boundary conditions obtained from an unspecified larger-scale simulation, such as continuum furnace-scale global-local simulation. The model treats the solid as a single crystal region containing point defects, defect clusters, excess Te, Te-particles, and dislocations/twins. It tracks defect evolution using defect nucleation, migration, growth and/or motion as functions of temperature, composition, stress, and strain rate imposed from the global-local simulation. The model treats the SLI as a moving interface using modified solidification mathematics accounting for interface morphology, species concentrations, melt velocities, and anisotropic SLI attachment kinetics.

Dislocations are present in the model consistent with computed thermal strains and strain rates as imposed by the global-local continuum models taking into account the furnace configuration and crucible materials. Slip plane partitioning of dislocations is accounted for. Te concentration is tracked using the equilibrium phase diagram as solute and treated locally along dislocation cores and twin boundaries. 
The model considers that point defects and extended defects, such as twins and dislocations, are created as the interface moves with suitable creation/annihilation rates for point defects at the moving interface. In the growing solid point defect clusters are tracked using appropriate computational methods for reaction-diffusion processes. Dislocation patterning due to slip and climb are treated using an appropriate modeling method, such as dislocation dynamics. The model treats Te ingress from the liquid according to accepted mechanisms, including precipitation from excess Te using the PTX phase diagram, dislocation core/twin boundary plane diffusion pathways, and direct capture at the moving SLI from growth instabilities. A schematic of such a model is shown in Figure 1 with some but not all of the typical problem conditions are illustrated here.

\subsection{Outline of Approach to Address the Computational Modeling Problem}

Although the issue of developing models and modeling approaches to crystal growth based on multiscale modeling approaches has been addressed [41, 42], we feel that this is inadequate. In particular, these methods do not contain sufficient detail that a mesoscale model of CZT growth, such as that discussed in Section 3.2, can be created containing the correct physical mechanisms. We are encouraged, however, by the work that has been done to model defects in $\mathrm{Si}$ [43], which can be used to guide new methods and approaches. Based on the prior work in this area and based on our understanding of the growth regimes of interest for CZT SLI computational modeling and simulation, we describe the modeling regimes for further discussion using the outline below. The computational modeling regimes are divided into 6 levels that all have appeared in the crystal growth modeling literature as either separate or coupled regimes.

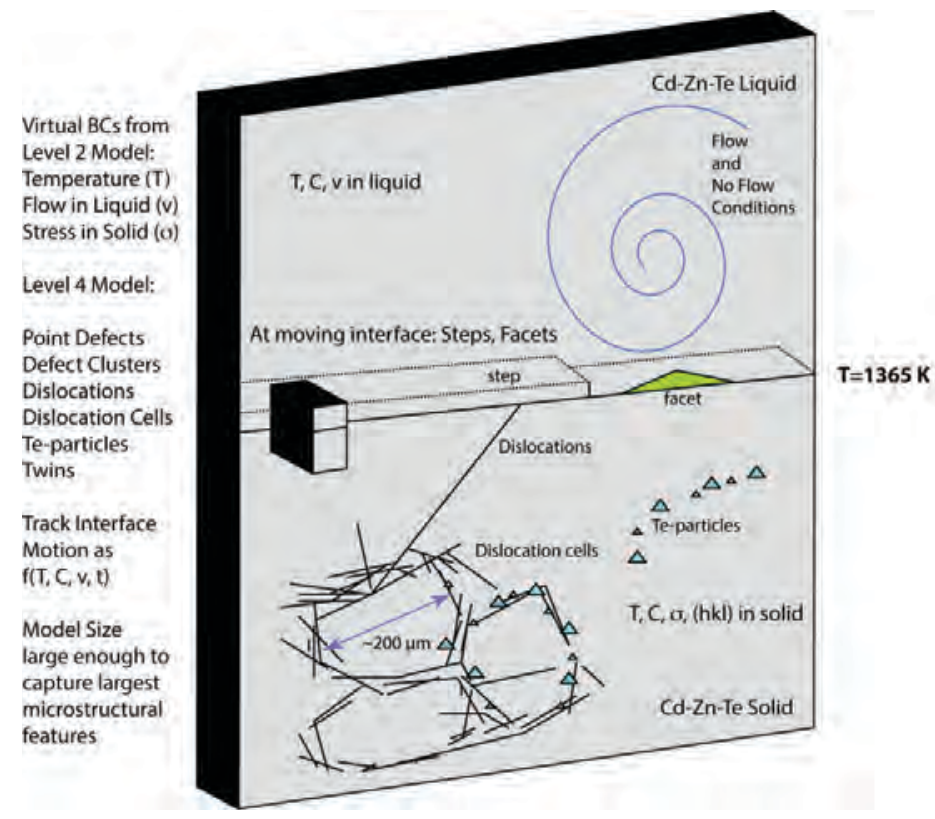

Figure 1. Schematic of a high-resolution 3D Level 4 model envisioned as the target model for CZT meltgrowth interface modeling. The model consists of a block of material at $1365 \mathrm{~K}$ with solid and liquid at equilibrium initially. The model has BCs from Level 2 and consists of a single oriented grain of CZT slowly solidifying from the melt. Point defects, point defect clusters, Te-particles, dislocations, and dislocation cells are tracked as a function of time and location. The SLI evolves with an interface morphology suitable to the crystalline orientation. SLI instabilities are treated and related to growth velocity, thermal gradient, flow gradient, stress, and liquid composition. 
1) The Level 1 model is a furnace-level global model of heat transfer, fluid flow, and radiative heating have been solved and/or could be solved for any particular furnace system to a high level of thermal accuracy [7] as indicated in Figure 2 below.

2) Embedded in this Level 1 model is a Level $\mathbf{2}$ model that is more local.

a) It couples to the global model BCs and is embedded self-consistently in the Level 1 model [7].

i) This Level 2 model has no crystallographic texture or orientation data.

ii) It has no grain morphology information.

iii) It allows S-L growth mechanics to be considered in a continuum sense.

iv) It can compute temperature, velocity, and compositional data at the moving SLI.
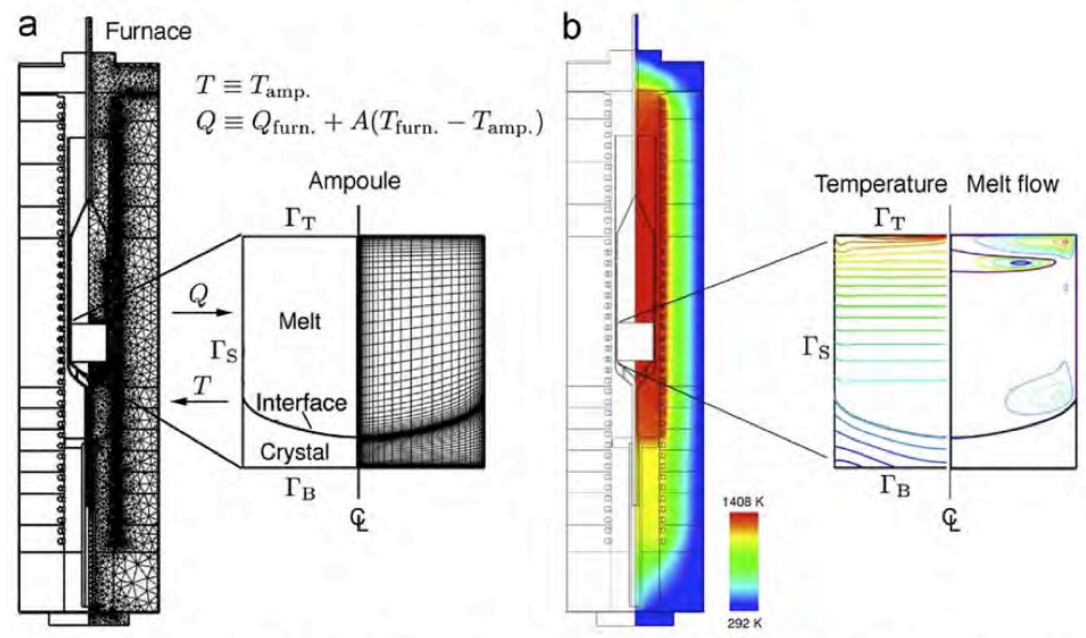

Fig. 1. Melt crystal growth in an electrodynamic gradient freeze furnace is represented using coupled models. (a) Matching temperature and heat flux boundary conditions are exchanged between a finite volume furnace model and a finite element model of ampoule contents. (b) Temperature in the furnace is computed by CrysMAS, and temperature and melt flow in the ampoule are computed by Cats2D.

Figure 2. A schematic view of Level 1 and Level 2 models showing a global and local coupled model from Ref. [7]. A furnace Level 1 model (large meshed region) is explicitly coupled to an ampoule region Level 2 model (inset). We envision that the Level 2 model supplies detailed boundary conditions for the Level 3 to Level 5 mesoscale models.

3) The Level 3 model is a multi-grain crystallization model coupled via the local fields such as the temperature, concentration, and melt convection fields to the Level 1 Global Model and Level 2 Local Model. The model will focus on the effect of local fields and SLI thermodynamic and kinetic properties on SLI morphological instabilities. The coupling to the Level 2 model is on a selfconsistent basis but this Level 3 model is not embedded directly within a Level 2 model. There is no direct coupling to the Level 1 model.

a) This Level 3 model focuses on exploring sensitivity of SLI morphology, connectivity to growth conditions, and creating a plausible database of SLI morphologies and growth kinetics based on SLI thermodynamic and kinetic properties and growth mechanisms and based on the local fields developed at the Level 2 model.

b) A variety of solidification and grain growth models are available to use at this level.

i) These include moving finite element (FE), phase field, level set, etc. 
c) This involves stochastic processes, such as the nucleation of steps and grains, and the fluctuations of local fields, and therefore cannot be deterministic entirely.

d) The Level 3 model does not deal with other crystal defects that we can term intra-grain defects.

e) The Level 3 model contains the SLI morphology, the grain morphology, grain orientation, and carries the continuum fields within it as discrete fields. These include elastic strain, temperature, composition, and interface velocities.

4) For the explicit model of defects we propose a Level 4 Model after [43]. The Level 4 Model is consistent with the Level 3 Model as follows:

a) Level 4 incorporates crystal defects within a single crystal lattice in each of the grains in the Level 3 model. This model can also consider a single grain only.

i) Solidification occurs due to a moving SLI interface that tracks growth rate, growth morphology at the SLI, defect creation at the moving SLI such as solute trapping, growth twin nucleation, and liquid phase variables ahead of the moving SLI, such as melt convection and composition.

ii) The morphology at the moving SLI interface is resolved at the correct length scale to capture physical growth instabilities, growth steps, and SLI roughness.

b) This Level $\mathbf{4}$ model focuses on exploring defect generation, the sensitivity and connectivity of defect generation to SLI morphology and growth conditions, and creating a plausible database of defect generation and growth kinetics based on SLI morphology and evolution kinetics from

Level 3 model and based on the local fields developed in the Level 2 model.

c) All necessary crystal defects are tracked in the solid behind the moving SLI.

i) Use reaction rate theory clustering methods to account for defect diffusion, annihilation, and clustering.

ii) Use classical and non-classical nucleation theory to account for the nucleation of defects such as Te precipitate, dislocation, and twins.

iii) Dislocation dynamics can be used to track dislocation patterning under thermal stress during cooling.

(1) Dislocation creation is based on physical models developed for semiconductors.

iv) The correct mechanisms for Te ingress, dislocation and twin formation are required for CZT and is still an active area of research.

5) The Level 5 models are the atomistic models that are used to develop kinetic physics models and parameters for the Level 3 and Level 4 SLI and defect model. This level appears to be required in order for level set, or phase field models to operate and define nucleation processes of defects and interface kinetics.

a) The kinetic properties of SLI, including defect nucleation barriers and defect mobilities, are important inputs to calculate the nucleation rate and model SLI morphology stability, and defect nucleation and growth kinetics in Level 3 and Level 4.

b) This model needs a large simulation system and long simulation times to capture the rare events with good statistical information. Coupling different atomistic methods may be required to accurately search the energy barriers of different kinetic processes.

6) The Level 6 models are also the molecular or atomistic model regimes that focus on calculating the necessary configurations of defects, defect energies, and thermodynamic properties of the system. This regime can make use of all suitable atomic methods.

a) The most accurate ab initio methods that we have available. 
b) Semiempirical atomistic methods including MD using developed interatomic potentials for CZT. See Figure 3 for example simulations using MD and models of SLIs of differing crystalline orientations.

c) Kinetic Monte Carlo methods to track defects and defect structures.

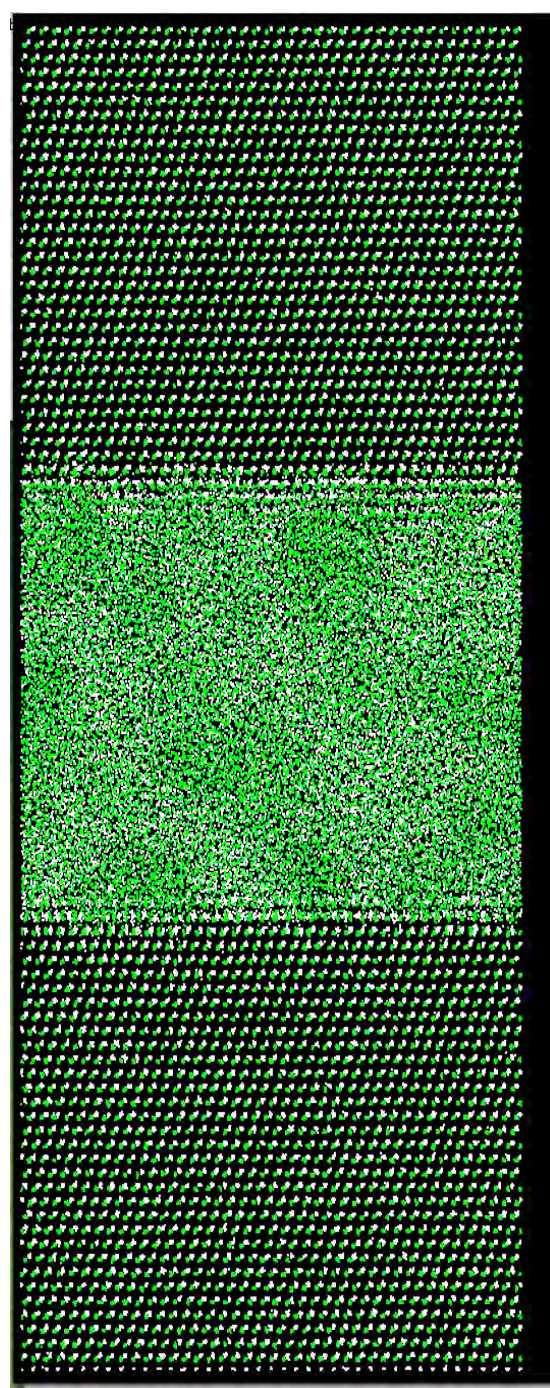

(a)

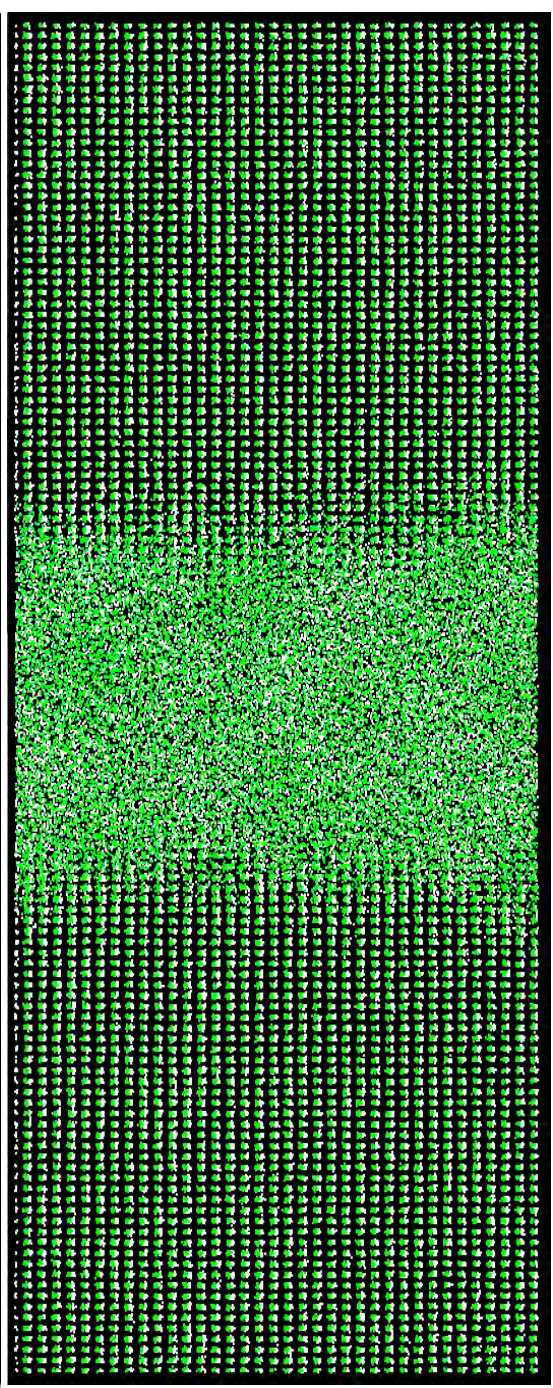

(b)

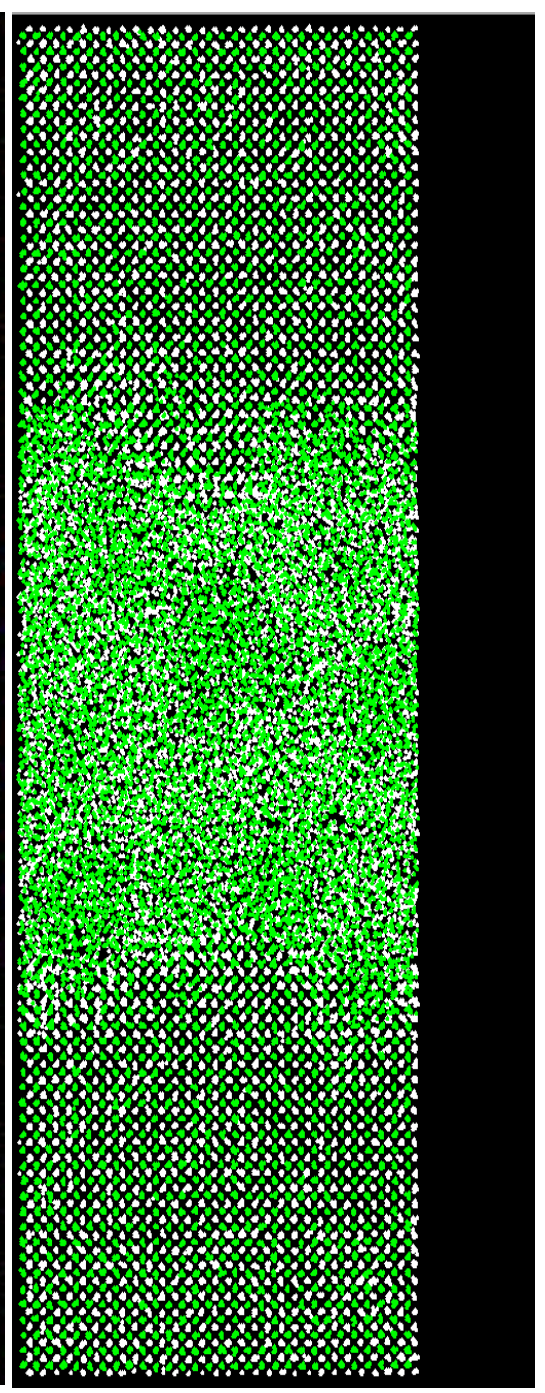

(c)

Figure 3. Atomistic models of SLI are shown at equilibrium for CdTe using PNNL interatomic potential as a function of interface orientation. Shown in (a) is a (111) interface normal showing smooth, flat interface structure. In (b) and (c) are shown (110) and (100) interface normals, respectively. Both (110) and (100) exhibit atomically rough interfaces with visible growth facets. 


\subsection{Assessment of the Current State-of-the-art for Crystal Growth Modeling - Methods and Approaches.}

\subsection{State-of-the-art Continuum Crystal Growth Models}

\subsubsection{Governing equations for continuum crystal growth models}

Although various numerical models were developed to simulate multiphase problems in recent decades, especially with the rapid development of computer techniques, traditional methods based on continuum material assumptions $[44,45]$ are still the most popular simulation tools and used for many different industrial areas because of efficiency, stability, and reliability. In continuum crystal growth models, the flow behavior in melt domains is governed by fluid mass and momentum conserve equations, such as Navier-Stokes (NS) equations [46, 47] and Boltzmann equations [48-50]. Navier-Stokes equations are the most widely studied method for solving fluid dynamic problems, and there are various numerical methods to solve it in different applications, such as finite difference (FD), finite volume (FV), and finite element (FE) methods. Because the Navier-Stokes equations are nonlinear, implicit numerical methods are highly recommended for avoiding the numerical instability [51]. Implicit schemes benefit the numerical NS equations solvers stability criteria at large time step intervals, but they also limit the parallel computational efficiency of the solver [52]. Boltzmann equations use distribution functions to represent fluid behavior, and can be solved by lattice Boltzmann methods (LBM) or lattice kinetic models (LK). One of the advantages of the lattice-based method is dealing with complicated or moving solid boundaries useful for crystal growth models. Another advantage is that LBM or LK methods were designed for high efficient parallel computation [53]. The fluid mass and momentum conservation equations are coupled with energy equations [54] to solve the heat transfer in both liquid and solid phase for crystal growth models. The energy equation can be numerically solved by the traditional methods, such as the FD, FV, and FE method, and it also can be solved in lattice-based schemes. The distribution of chemical components, such as zinc in CZT or Ga in GaSe, in liquid phases is described by a convective diffusion equation, and the fraction in solid phase is governed by a diffusion equation [46, 47]. The convective diffusion and diffusion equations can be solved by the traditional FD, FV, and FE methods or in latticebased schemes as well. Lattice-based schemes can significantly increase the computational efficiency in massive parallel computational systems, while the implicit traditional methods enable solver stability at large time step intervals.

\subsubsection{Methods for simulation of liquid-solid phase separation}

The continuum crystal growth models require determination of the interface coupled with fluid dynamic and heat transport solutions. There are several approaches to simulating and tracking the liquidsolid interface, and they can be divided into three categories: the front-tracking method, front-capturing method, and multi-phase LBM. Front-tracking methods directly "track" the location of the liquid-solid interface, so they allow more accurate calculation of the curvature of the interface $[55,56]$. Although front tracking methods require generating a dynamic mesh, which is always a computationally challenging task, front tracking methods are widely used in continuum crystal growth models [8, 47], because the movement of the SLI is very slow [57]. For increasing the efficiency of dealing with deformation of liquid and solid domain dynamically, H. Lee and A. J. Pearlstein [47, 58] transform the dynamic computational domain to a fixed one, and all the numerical simulation is implemented on the fixed mesh. The deformation of real liquid and solid domain only changes the coefficients for the domain transformation between real computational domains and fixed domains, instead of changing the shape of 
meshes dynamically. Front-capturing method uses a single continuum field with discontinuous properties at the SLI to separate the liquid and solid phases. The most widely used front capturing methods are the Marker-and-Cell (MAC) method [59], Volume-of-Fluid (VOF) method [60], level set method [61], and phase-field method [62]. Among these methods, the phase-field method has become a standard tool to track free-boundary problems and simulate interfacial pattern formation phenomena in solidification and other systems [63-65]. The phase-field method avoids explicit front tracking by replacing sharp interfaces with spatial-smoothly diffused boundaries between bulk phases. Thus, the phase-field method is suitable for simulating time-dependent free-boundary problems, especially when complex geometries are present $[66,67]$. Multi-phase LBM have been developed as multiphase numerical simulation tools for crystal growth and phase separation in recent decades [68,69], and incorporate inter-particle forces/microscopic interactions into the fluid distribution function, without tracking/capturing the interfaces [70]. There have been a number of multi-phase LBM presented in the literature, and the Shan and Chen (SC) model [71$74]$ is one of the most widely used, which allows phase separation to occur automatically when the interaction potentials are properly chosen. There is also the so-called free-energy-based approach proposed by Swift et al $[75,76]$, which incorporates free energy function into the LBM model.

\subsubsection{Applications of continuum crystal growth models}

Continuum crystal growth models are widely studied and used to for investigating the performance of solidification systems, such as ampoule tilting or imperfection effects, accelerated crucible rotation, and magnetic field effects. Figure 4 shows the simulation results from a continuum crystal growth model that coupled a LBM fluid flow solver with a phase-field interface capturing method. Figure 4(a) shows the streamline in a tilted ampoule, and Figure 4(b) shows the streamline in ampoule with accelerated rotation.

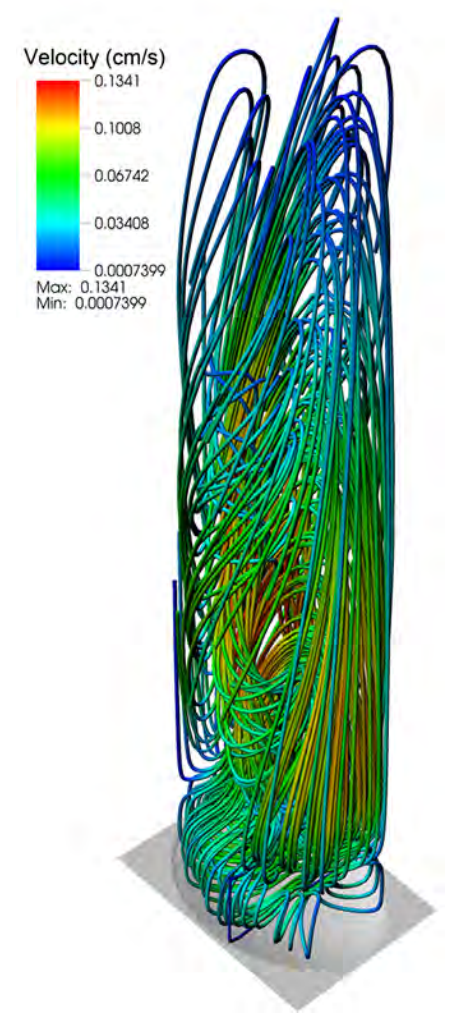

(a)
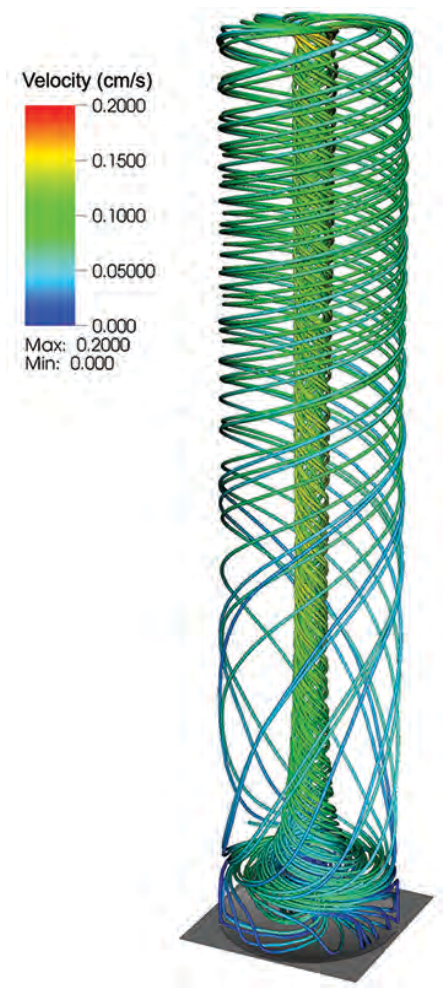

(b)

Figure 4. Streamline in liquid phase, (a) tilted ampoule, (b) accelerated rotating ampoule. 
Xiao et al [46] were among the first to compute crystal growth in a vertical solidification system whose axis is tilted away from the gravitational vector. They showed that the flows generated by tilting leads to better mixing of solute and could reduce radial segregation in the melt. L. Lun et al [20] shows that a 20 degree tilt angle can reduce the zinc concentration standard deviation to a minimum in CZT.

Accelerated crucible rotation technique (ACRT) [16, 17, 77-80] has been used in crystal growth for many years; the purpose of acceleration being to maximize convective flow by generating Ekman boundary layers at solid surfaces [81]. A series of experimental studies of ACRT has been conducted by Caper and coworkers [77-80], and they observed Ekman flow and a sidewall instability, which is identified as a Couette instability. A. Yeckel and J. J. Derby [54] used continuum crystal growth models to numerically simulated the ACRT applied to the vertical solidification system, and sidewall instability was shown in their results and identified as a Taylor-Görtler boundary layer instability [82]. It was shown that changing of acceleration in the rotation cycle can dramatically affect the radial and axial segregation in crystal growth system [83]. The rotation, acceleration, and deceleration lead to complicated turbulent fluid structure, such as boundary layer instabilities mentioned above, and the theoretical analyses of turbulence in crystal growth system is very limited. From the view of continuum crystal growth models, applying different turbulence models to solve the fluid phenomena in liquid phase will help build a more accurate and reliable numerical tool for study the crystal growth system with rotation, which will lead to a better understanding of the relationship between the crystal growth quality and rotation speed.

The traveling magnetic field (TMF) is a direct way to counteract buoyancy forces $[84,85]$ that are often responsible for introducing undesired effects at the growth interface [86] due to buoyancy driven convection. Another application of magnetic fields is the use of a rotating magnetic field (RMF) that creates a swirl to counteract the convection caused by buoyancy [87]. The Lorentz force imposed by the magnetic field is solved using Maxwell's equations, and is applied on the fluid field as an external force or body force. Through continuum crystal growth models' simulation, A. Yeckel and J. J. Derby's work [12] shows that the magnetic field cannot significantly impact the convection because the low electrical conductivity of CZT $\left(\approx 10^{4} \mathrm{~S} / \mathrm{m}\right)$, while S. Yesilyurt et al. [86] shows that the tangential shear at the liquidsolid interface is reduced to near zero by the TMF with proper strength of magnetic field for a crystal with higher electrical conductivity such as Ge and GaAs, which can increase the quality of crystal growth.

Although the continuum crystal growth models are widely used and are reliable numerical tools for studying and designing solidification systems, there is a lack of systematic study of turbulent flow structures in the liquid phase. Validating and comparing the accuracy and efficiency of different turbulence models coupled to crystal growth models are needed for more reliable numerical tools for crystal growth models.

\subsection{Fine-Scale Growth Models (Mesoscale Models)}

Fine scale growth modeling (mesoscale modeling) refers to modeling crystal growth by coupling microstructure evolution and local continuum fields, such as melt convection, temperature, and concentration near the SLI. During the past four decades different mesoscale methods have been developed to study the microstructure evolution during solidification and crystal growth. However, due to large differences in length scales between the entire furnace and the microstructural features at the SLI, and large differences in time scales among macroscale fields, such as local melt convection near interface and interface evolution kinetics, most mesoscale simulations are quasi-stationary, i.e., the macroscale fields are usually used as the boundary conditions for the mesoscale modeling. The mesoscale modeling is also treated with a one-way coupling in most cases; the temperature, convection and concentration 
fields are transferred from macroscale to the local scale but there is no influence of local microstructure and field evolution on the macroscale fields as there would be in a fully self-consistent approach. Furthermore, most mesoscale models are phenomenological based that capture the morphology of the SLI, but cannot capture defect generation, such as dislocations, growth/deformation twins, Te-particles, and fluctuation-based interface morphological instability, which usually take place in mesoscale from nanometers to micrometers. Coupling microstructure evolution, local fields, and defect generations is at its very early stage but is paramount to understand the mechanisms of defect generation and the effect of furnace growth conditions on defect generation, crystal growth kinetics, and crystal properties.

Mesoscale models act as the bridge linking atomistic and macroscale information. The development of predictive mesoscale models relies on enormous knowledge that includes phase stability, defect energies, defect structures, defect mobilities, and local fields. During last half-century atomistic models, such as first principle calculations, MD, and kMC approaches have been successfully used to calculate the configurations and energies of defects, track interactions and motion of individual atoms, and study the atomic mechanisms behind different material processes such as diffusion, phase transitions, and deformation. At the macroscale, as reviewed in Section 4.1, heat transfer, melt convection and concentration fields can also be efficiently simulated. With the accumulation of thermodynamic and kinetic properties from atomistic simulations, experiments and macroscale modeling, an opportunity opens up for developing physics-based predictive simulation tools at the mesoscale to understand the mechanisms of defect generation, to predict three-dimensional microstructure evolution kinetics, and to provide insights for controlling the crystal growth.

This section is organized as follows. In Section 4.2.1 we will review observed interface instability, the factors affecting the interface instability and theoretical analysis. In Section 4.2.2, we will discuss the effect of interface morphological instability on defect generation and theory analysis. In Section 4.2.3 we will discuss current mesoscale modeling methods, and we will discuss the challenges and research directions in Section 4.2.4.

\subsubsection{Morphological Instability of SLI during Crystal Growth}

It is generally observed that a convex SLI favors single crystalline growth while a concave interface results in the growth of polycrystalline ingot. The ideal growth interface is flat for the growth of single crystalline ingot. Therefore, controlling the morphology of the SLI during unidirectional growth processes is crucial to obtain high quality crystals because it affects the defect and grain microstructures, and eventually the mechanical, optical and electrical properties of materials [88-91]. The questions are 1) What growth conditions control the SLI morphology?; 2) What are the mechanisms behind the interface morphological instability?; and 3) Can simulation tools predict the complicated interface morphological instability, and provide the insights to control the interface morphology? Answering these questions is important to science as well as to engineering technology and the crystal growth industries.

The SLI essentially consists of different orientated atomic planes. According to Jackson's theory [92, 93], the atomic planes or interfaces can classified using two types of interfaces. One type of interface, called facetted, such as (111) in Si and CdTe, has low interfacial energies and is usually atomistically smooth. Another type of interface, for example (100) in CdTe and Si, has higher interface energies and is usually atomistically rough. MD simulations by Landman et al. [94] and Abraham and Broughton [95] show the equilibrium (111) interface to be sharp and the (100) interface to be diffuse. There are extensive experimental and theoretical investigation on the interface growth mechanisms and morphological instability. Work at PNNL shown in Figure 3 supports this general result. 
Faceted interfaces grow layer-by layer by a lateral growth mechanism [96], which proceeds through the incorporation of adatoms into atomic steps; adatoms are incorporated at the step edges; and thus steps advance across the SLI, as shown schematically in Figure 5. On a vicinal interface, steps advance one after another in step flow. Ledge growth and step flow processes have been observed in real time by high resolution TEM [97] and MD simulations [98]. If the impinging flux is too high or the terrace size is too large (a low nucleation rate of steps and/or high surface diffusivity), two dimensional islands are nucleated first, but subsequently these islands also expand by step flow growth. Blasé and Zangwill [96] theoretically analyzed this wandering step flow growth and showed that there is a possibility of morphological instability when there is an imbalance in the attachment to steps of adatoms from the upper and lower terraces. If there is a larger surface flux of adatoms from the lower terrace than that from the upper terrace, step fluctuations are amplified during growth, resulting in a waveness or wandering of steps. This unstable growth regime is located between stable step flow and 2D nucleation in a phase diagram of the growth mode as a function of impinging flux and as a function of step spacing.

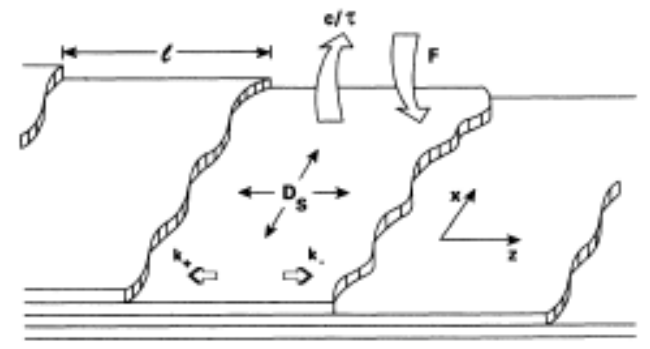

Figure 5. Schematic view of a vicinal surface during step flow growth [96]

Solute trapping at the SLI is a common phenomenon observed during rapid solidification. It is described theoretically in terms of a superposition of two reactions at the interface, the attachment of atoms independent of species at lattice sites, and solute-solvent redistribution across the interface by interdiffusive process. The ratio of solute concentration in the growing solid to that in the liquid at the interface is known as the nonequilibrium partition coefficient, which depends on the SLI velocity. Experiments demonstrated that that a greater degree of solute trapping at (111) is due to the lateral speed of (111) ledges for a (111) interface being greater than the lateral speed of ledges for (100) interface moving at the same velocity [99]. The effect of solute trapping on interfacial undercooling and growth kinetics of flat plane is analytically analyzed [100]. It is predicted that solute trapping causes significantly interfacial undercooling compared to solidification without solute trapping. In ordered intermetallic compounds, disordering trapping has been predicted and observed in the rapid solidification [101]. In systems that undergo either solute or disorder trapping, a transition from short-range diffusion-limited to collision-limited growth occurs, which originates in the reduced driving free energy for the formation of such metastable materials, resulting in three orders of magnitude change in the interface mobility.

The effect of anisotropy of interface kinetics on the stability of step flow forming a vicinal interface was studied [102] and it was found that step motion on a growing vicinal interface can induce its own stabilization with respect to the formation of step bunches. This kinetic stabilization results from the motion of the steps relative to a stagnant solution. Kinetic stabilization perturbation occurs for higher growth rates and smaller perturbation wavenumbers, whereas conventional capillarity-driven stabilization occurs for larger wavenumbers.

The effect of a parallel shear flow and anisotropic interface kinetics on the onset of (linear) instability during growth from a supersaturated solution is analyzed including perturbations in the flow velocity 
[103]. It is found that a shear flow counter to the step motion (positive shear) enhances stability. For sufficiently large shear rates the interface is morphologically stable. For sufficiently low supersaturation, the interface is stable for positive shear and unstable for negative shear. A critical negative shear rate is also found for which the interface becomes unstable as the magnitude of the shear rate increases.

Morphology instability of atomic step motion was observed on Si (111) surface in situ [104]. Step wandering was observed when the surface flux to or from the terrace preceding a step dominates over the surface flux to or from the terrace that lags behind the step. The wavelength associated with the wandering of an initially straight step is measured as a function of the impinging flux. The amplitude of wandering for a circular step is measured as a function of the terrace size. These quantities showed reasonable agreement with the predictions from linear stability theory.

Rough interfaces with high interfacial energies will transform to a zigzag faceted interface bounded by low interfacial energy (111) planes. Because the (111) planes typically have the lowest surface energy, a zigzag-faceted interface bounded by (111) planes is expected to form at the SLI of rough growth planes. As one possible formation mechanism of a zigzag-faceted interface, it was considered that atomic-scale facets are initially formed, which gradually enlarge to macroscopic facets during crystallization. Recently, the zigzag-facet formation at the Si (100), (110), and (111) SLIs has been investigated by in situ observations $[105,106]$. In the case of the $\mathrm{Si}(110)$ and (112) SLIs the morphological transformation from planar to zigzag was observed at high growth velocities, however, a planar interface was maintained at the Si (111) interface. Figure 6 shows that temperature perturbation can cause SLI morphological instability. The results demonstrated a universal behavior that a zigzag faceted SLI is formed when the temperature gradient at the interface becomes negative at a high growth velocity over a critical value.

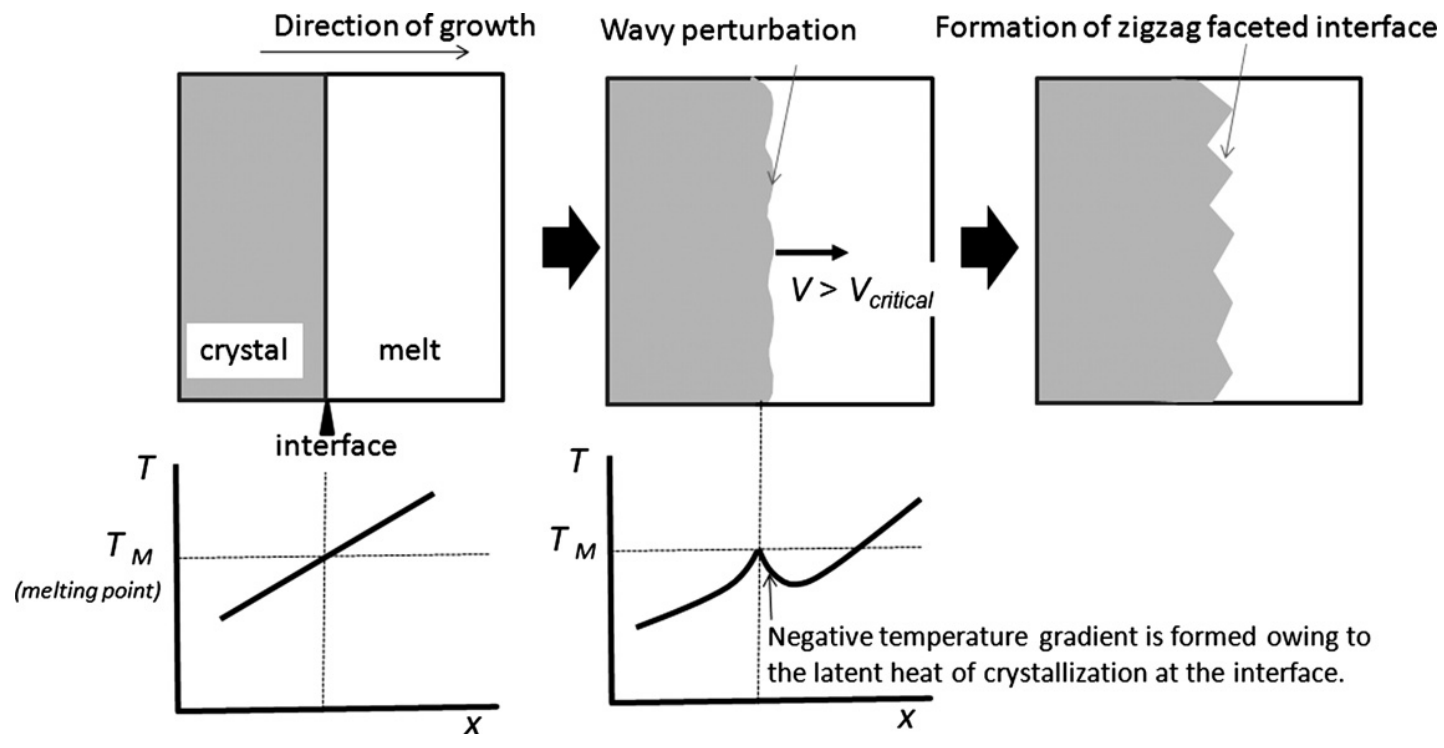

Figure 6. Schematic illustration of an SLI and the thermal field at the SLI when a zigzag faceted interface is formed during unidirectional growth. Initially the temperature gradient was positive in the growth direction because crystal growth was initiated from an unmelted seed crystal. A negative temperature gradient formed locally in the Si melt owing to the latent heat when the growth velocity is higher than its critical value.

In alloys the inhomogeneous solute segregation induced by natural convection and melt solid interface shape [107] causes an inhomogeneous supercooling at the SLI, i.e., a temperature perturbation at 
interface. Hurle et al. [90] proposed a formation mechanisms of faceted interface due to constitutional supercooling by this process: lateral segregation and micro-facet formation. This is consistent with the temperature perturbation mechanisms observed in Si [106]. There are a number of experimental observations the effect of applied magnetic fields [14, 108-110] and growth rates [111] on interface morphological instability. It is found that growth rate affects the growth direction [111]. At low growth rate, germanium grows ahead of the average solid-liquid interface, in the (100) direction. At high growth rate, the solidification mode changes, and grows in $<211>$ directions. Different growth direction causes different twin structures. Applying a magnetic field is an effective method to improve the growth interface and the quality of crystal during solidification. Oscillatory convection in a moderate length cylindrical cavity with the Bridgman method could be damped by the action of a vertical magnetic field [112]. It was found that under microgravity conditions rotating magnetic fields could be used to overwhelm residual buoyancy induced convection and to control the uniformity of the solution zone composition at the growth front without appreciable modification of the growth interface shape by means of numerical simulation of THM growth of CdTe [109, 113].

In summary there are a number of factors such as capillarity, temperature gradient, melt convection, solute trapping, solute segregation, growth kinetic anisotropy, growth rates and applied magnetic fields which affect the morphological instability of a typical SLI during crytal growth. The morphological instability of an SLI could be categorized into three types, i.e., constitutional supersaturation instability, crystallization flow induced local instability, and microscopic growth mechanism induced instability, which were also recognized by scanning electron microscopy observation of the CdTe crystals [114]. All interfaces could be described by (111) steps, but step density and step growth kinetics depends on the interface orientations. To predict the interface growth kinetics and interface morphological instability, we need to understand the effect of growth conditions such as local concentration, temperature, melt convection, and stresses on the nucleation rate of steps and impinging flux.

\subsubsection{Defect Generation during Crystal Growth}

Once the morphology of flat interfaces becomes unstable, the crystal grows in different modes. Cellular growth, zigzag growth, growth direction changes, and new grain nucleation and growth are often observed in experiments. Different growth modes may be accompanied by the formation of different defects such as inclusions, growth twins, dislocations, deformation twins, and grain boundaries. Experiments suggested that interface morphological instability not only affect the SLI shape, but also defect generation during crystal growth, therefore, the material's mechanical, optical and electrical properties.

Almost all of the detector-grade CZT produced today is grown from Te-rich melts and the resulting ingots contain large concentrations of Te-particles, as inclusions or precipitates $[88,115,116]$. The typical diameters of Te-particles formed in CZT are in the 1 to $50 \mu \mathrm{m}$ range. The average diameter of Teprecipitates is 10 to $30 \mathrm{~nm}$. It is believed that inclusions originate from morphological instabilities at the growth interface $[39,91]$. As micro-facets form and grow on the interface, cellular interface structures form that depend on the SLI orientations and local growth conditions. The faceted SLI traps excess solutes via a direct capture mechanism. As a result, Te-rich melt droplets are captured from the boundary layer ahead of the SLI. The pattern of Te-particles depends strongly on both the SLI morphology (which is dependent on the SLI orientation) and on the solute concentration near the SLI. Figure 7 shows regions of as-grown CZT and CdTe SLIs. The brighter portions are the Te-rich alloy captured by a quench to freeze-in the SLI structure, while the dark regions are the as-grown CZT or CdTe solid [115]. Trapping of 
Te-rich droplets near the interface and, hence, formation of Te-particles in CZT or CdTe due to irregular interface is clearly evident from Figure 7.

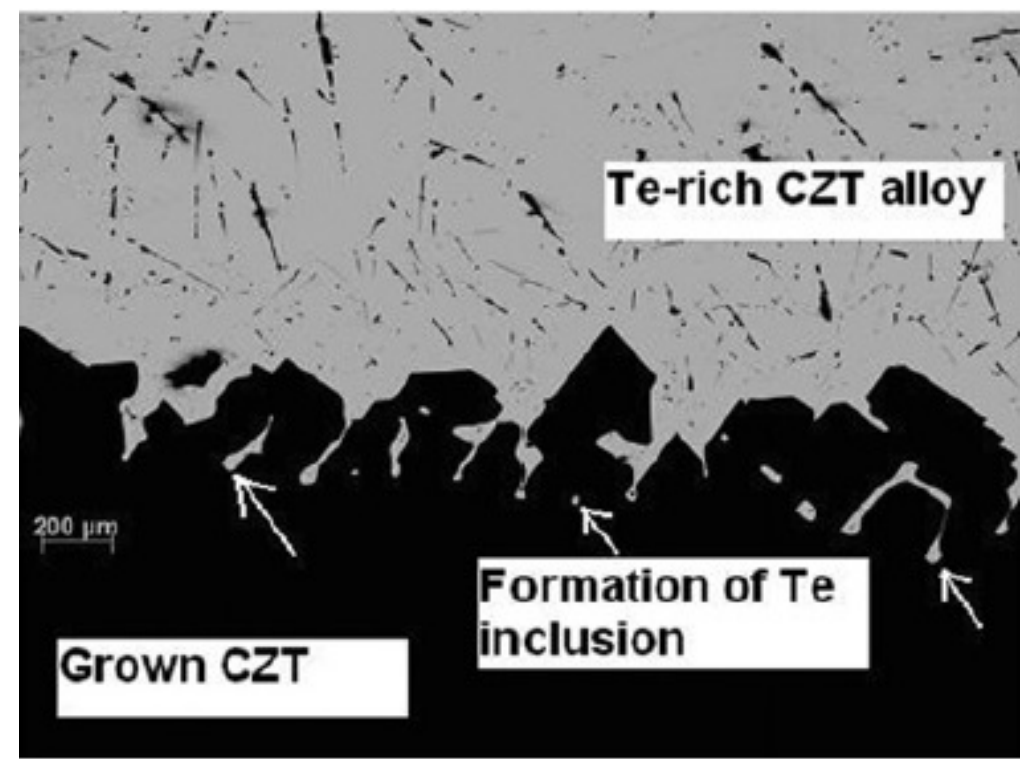

(a)

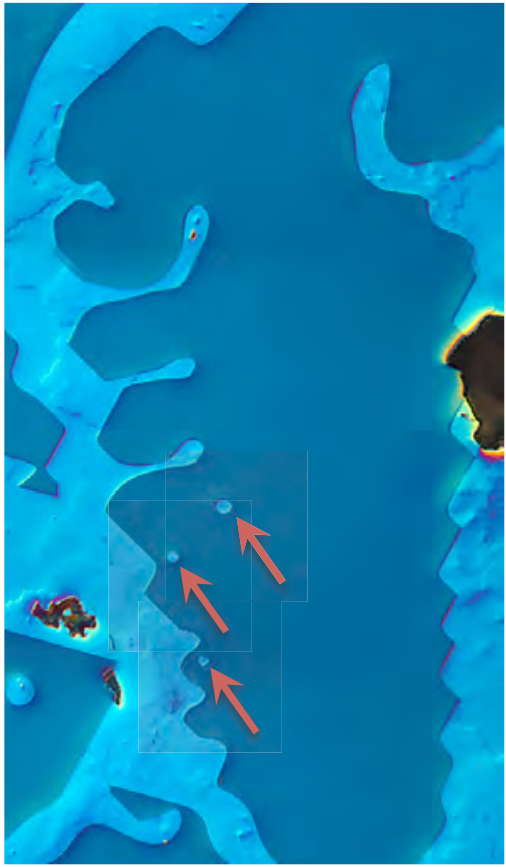

(b)

Figure 7. (a) Te-inclusions formed near a CZT growth interface [115] and (b) CdTe growth fingers (dark blue) in Te (light blue) showing similar crystallographic instabilities and Te inclusions (PNNL). The CdTe growth fingers in (b) are oriented in the (110) crystallographic direction, which is the fast growing direction for CdTe. The growth fingers pinch off Te-droplets that become Te-particles.

Due to the retrograde slope of the solidus line in Cd-Te phase diagram [116] and Te segregation on defects such as grain boundaries, twin boundaries and Te-rich inclusions, small precipitates are formed during cooling. The nucleation and growth of Te-precipitates is controlled by atomic diffusion. Specific sites along the grain boundaries and twin boundaries were seen to act as preferential nucleation sites (i.e. triple junctions of grain boundaries, jogs in the twin boundaries) [117-120]. Experiments show good correlation between the spatial distribution of Te-particles and existing dislocation networks (chuck's work).

Extremely low critical resolved shear stress (CRSS) near the melting point is an intrinsic property of zincblende structures. For example, the CRSS for CdTe is about $0.2 \mathrm{MPa}$ above $1273 \mathrm{~K}$. This implies that the nucleation of dislocations has very low barrier, evidenced by the fact that it is almost impossible to grow big dislocation free CdTe crystals. Experiments show a massive increases in dislocation density after the formation of a cellular interface due to the stresses associated with the crystallization following migration of the trapped liquid droplets [121]. Dislocation emission from large Te-particles is also observed [88]. In addition, crystal growth under industrial conditions often involves large thermal gradients and thus generates thermal strains and residual stresses during cooling and/or in the final product. These stresses may cause dislocation nucleation and motion, and dislocation network formation [122]. Combining the thermal stress in a cylindrical growing crystal with the basic $<110>(111)$ glide system of the zincblende structure the Schmidt contour can be theoretically calculated [123] and shows that the stress relaxes by radial glide along $<110>$ directions. This was very clearly demonstrated 
theoretically and experimentally for GaAs by Jordan et al. [124]. Billig discovered that dislocation density of Ge crystal was correlated with the imposed temperature gradient [125]. A theoretical formula was developed to estimate the critical temperature gradient which is needed to exceed the CRSS for dislocation multiplication in III-V and II-VI compounds [126]. It is found that the critical temperature gradient is 1 to $2 \mathrm{~K}$ per crystal length compared to the crystal diameter. Hence controlling the temperature field at all stages of crystal growth is of essential significance.
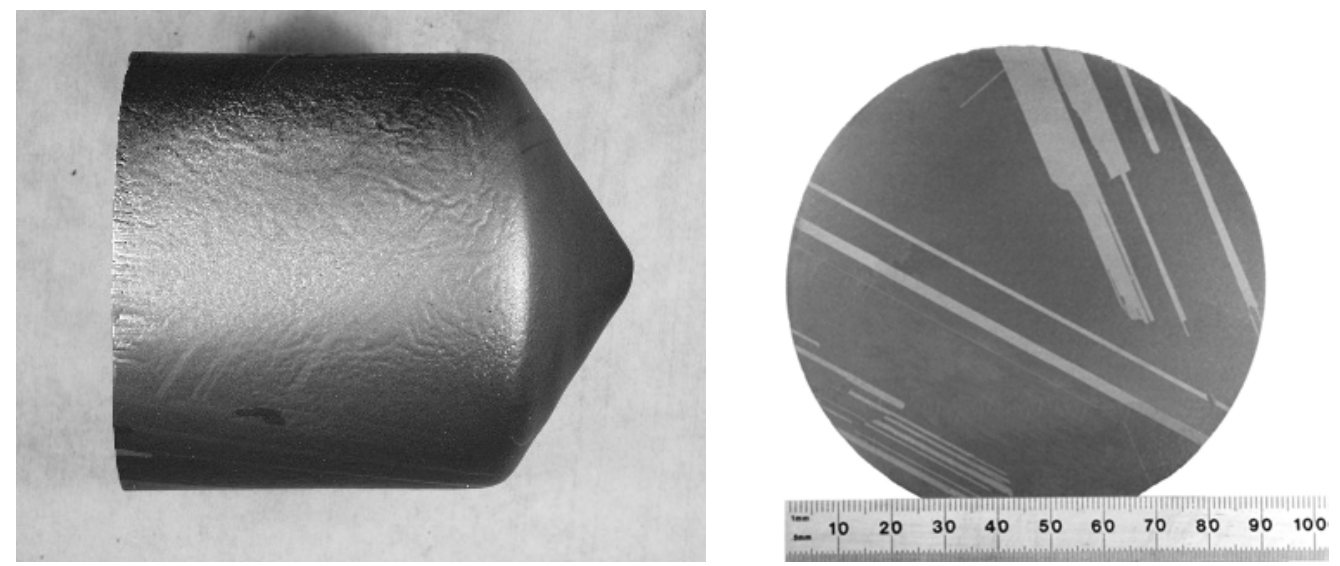

Figure 8. Photograph of a 92-mm diameter 4-kg CZT ingot grown by the EDG technique using Cd partial pressure control and a radial slice of the same [88].

The stacking fault energy in zincblende structures is also very small. The first principle calculation show that the stacking fault energy is about $0.1 \mathrm{~J} / \mathrm{m} 2$ for CdTe. Therefore, the twin is common defects in growth crystal with zincblende structures [90, 127-134]. Figure 8 shows the twin structures observed in CZT. Twin formation in the zincblende lattices, which is closely related to interface morphological instability: facet formation is specified by a rotation of the lattice by 60 degree about a $<111>$ axis, the twin lying on the orthogonal (111) plane. Hurle [127] proposed a mechanism of twin formation during Vertical Bridgman Growth, which suggested that when facets can intersect the three-phase boundary (TPB) (crystal, melt and ambient) twin nucleation might take place preferentially at that boundary. It also found that a twinned nucleus is thermodynamically favored when the supercooling exceeds a critical value given by $\delta T_{c}=\left(\sigma_{T} T_{m} / h \Delta H\right)\left[A_{e} /\left(A_{e}-A_{e}^{0}\right)\right]$ where $A_{e}$ is the work of formation of a truncated nucleus intersecting the TPB, $\Delta H$ is the latent heat of fusion, and $\mathrm{H}$ is the height of the step. Figure 9 shows another model of parallel-twin formation at crystal-melt interface during crystal growth. When a twin boundary is accidentally formed on a (111)-facet plane, another twin boundary is formed parallel to the first twin after lateral growth is promoted $[89,135]$. Twin nucleation at TPB was observed $[89,127$, 136, 137]. Experiments show the influence of polarity on twinning [138]. Like dislocations, the residual stresses associated with temperature gradient and internal stresses around defects may also induce the twin nucleation and growth [133, 134, 139-144]. 


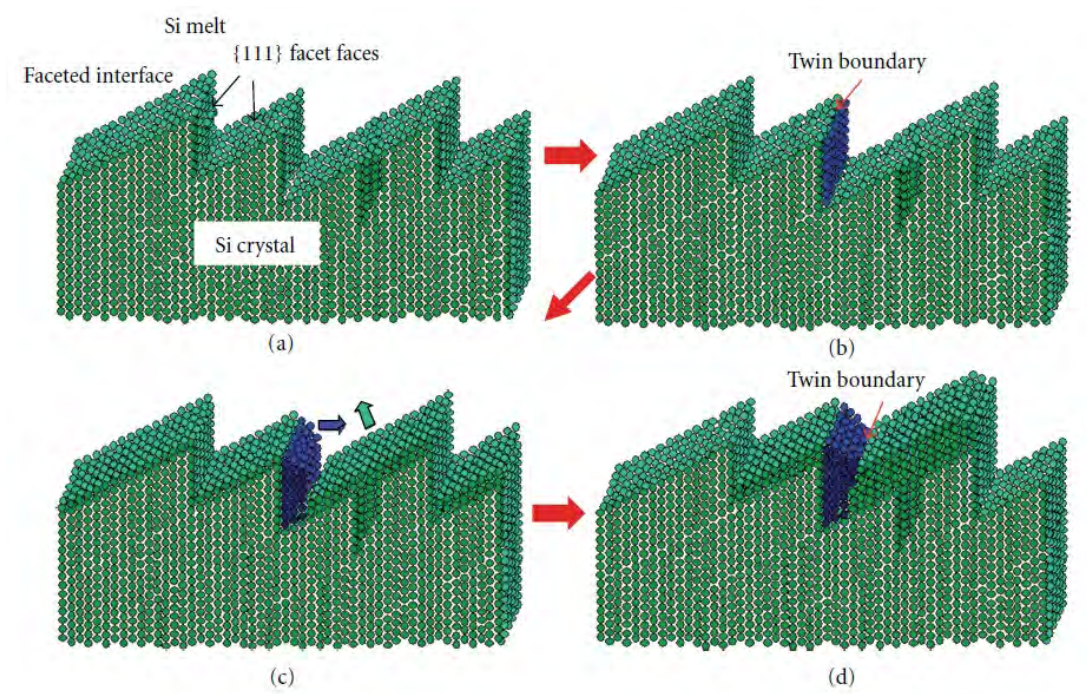

Figure 9. Model of parallel-twin formation at (111) faceted crystal-melt interface during crystal growth.

In summary, the formation of defects is directly related to the interface morphology. Therefore, it is crucial to understand the effect of growth conditions, which include the seed crystal orientation, conical growth angle, temperature gradient, composition gradient, temperature and composition fluctuation, facets size, melt supercooling, melt convention, and applied magnetic fields, on interface morphological instability, interface morphology evolution of unstable interface, and defect nucleation and growth in order to made high quality crystals.

\subsubsection{Mesoscale methods of crystal growth and defect evolution}

Mathematically microstructure evolution can be formulated as moving boundary problems. Historically these "Stephan problems" or moving boundary problems have been challenging from a computational point of view, but recently a number of new computational methods have been developed to numerically solve them. According to the description of interfaces, these methods can be classified as sharp interface and diffusive interface methods. The sharp interface methods including the front-tracking [145], volume-of-fluid [146], and level set [147] methods need to track the interface during simulations. The front-tracking algorithm can be used in conjunction with adaptive mesh refinement near the interface, but it is difficult to apply it to dynamic interfaces that undergo complicated topological changes. Volumeof-fluid methods have the advantages of conserving mass by explicitly tracking volume fractions for each cell, but reconstruction of interfaces from volume fractions and calculation of associated geometric quantities, such as the interface curvature are not straightforward. This is particularly true in the 3D simulations. The level set method can easily handle complex geometries, but it suffers from mass loss/gain problems if more complicated methods based on the volume-of-fluid or front tracking approaches are not implemented. The diffuse-interface method such as the phase-field approach does not require explicitly tracking the moving interface. The approach, originally developed by Cahn and Hilliard [148] in the 1950s, is based on the concept of a diffusive interface that can be defined in terms of a density, structure or composition field (i.e., a phase field) that changes smoothly from one phase to the other over an interface zone with a non-zero width, $w$. In this manner, numerical difficulties associated with the boundary conditions at the sharp interfaces are avoided, and no explicit interface tracking/capturing is required. The microstructure evolution is described by diffusive relaxation (Cahn- 
Hilliard [148]) of conservative phase fields or direct relaxation (Allen-Cahn [149]) of non-conservative fields. The main advantage of the phase-field method is the fact that it can model complicated microstructures with multiple phases and defects without prior assumptions. Furthermore, it is possible to incorporate the contributions of temperature gradients, melt convection, and long-range elastic interactions due to formation volumes of defects.

The phase-field method has become a powerful tool to simulate microstructure evolution in materials [150], a subject of both fundamental and applied interest [151], and more generally used to tackle freeboundary problem. In principle, simulating the evolution of complex morphologies in two and three dimensions is straightforward with phase-field method $[150,152,153]$. However, for a material process involving mass and heat transfer, making quantitative predictions on experimentally relevant length and time scales [154] is a challenge. This challenge stems from the fact that important model parameters such as the width of the diffuse interface and characteristic dissipation time scales in phase-field simulations is simply not feasible in order to describe the thermodynamic and kinetic properties of a real physical system. With such a set of parameters, both the width $W$ of the diffuse interface and the characteristic dissipation time $t$ in the phase field kinetics are usually microscopic: $W$ is a few angstroms and $t$ is roughly the ratio of $W$ and the thermal velocity of atoms in the materials. In contrast, diffusive transport of solute in bulk phases occurs on macroscopic length and time scales that are several orders of magnitude larger than $W$ and $t$, respectively. Therefore, resolving both microscopic and macroscopic length/time scales in phase field simulations for typical experimental length and time scales is impractical, even with efficient algorithms. During past two decades, great progresses have been made in quantitative phase field simulations of microstructural evolution in solidification [154-157]. Atomistic simulations make it possible to provide accurate thermodynamic and kinetic properties such as interfacial energy of melt-crystal interface [158-162], crystalline anisotropies [163-165], interface kinetic properties [166-169], and crystal nucleation [170-175]. The availability of these properties is critical for quantitative modeling of solidification microstructures. Quantitative phase-field models [154-156] have been developed which can use large length and time scales without loss of interface physics. Melt convection is taken into account in phase-field models by coupling lattice Boltzmann method to efficiently solve the fluid flow [176-178]. The detail on the progress of quantitative phase-field models can be found in review papers $[154,157]$.

\subsubsection{Mesoscale modeling of interface morphological instability}

Experiments and theory show that many factors, such as anisotropy of interfacial energy and mobility, solute trapping, temperature gradient, concentration gradient, melt convection, and defect nucleation, affect the interface morphological instability. Interfacial properties can be calculated by atomistic simulations such as first principle calculation, molecular dynamics and kinetic Monte Carlo simulations. Compared with sharp interface methods, one advantage of phase-field models is that it is able to take into account any complex anisotropic interfacial properties which are important for correctly describing new phase nucleation and the equilibrium morphology of microstructure as well as the microstructure evolution kinetic. For cubic symmetrical crystal such as fcc, atomistic calculations have established that accurate parameterization of interfacial energy anisotropy $\gamma(\hat{\mathbf{n}})$ generally require two anisotropy parameters associated with both four- and sixfold anisotropy terms:

$$
\gamma(\hat{\mathbf{n}})=\gamma_{0}\left[1+\varepsilon_{1} K_{1}(\theta, \phi)+\varepsilon_{2} K_{2}(\theta, \phi)\right]
$$


where and are the spherical angles of the interface normal, $\gamma_{0}$ represents the orientation-averaged interfacial free energy, and and parametrize the strength of the four and sixfold anisotropy cubicharmonic functions and [163]. This anisotropic interfacial energy is widely used in phase-field models for predicting dendrite growth during solidification [157]. Due to slow growth rate during unidirectional crystal growth, faceted interfaces and sharp corners are often observed in SLI. This means that interfacial energy is a function with cusps or non-convex. With the classical interfacial energy formulation, the phase-field evolution equation becomes ill-posed for the sharp interface case (i.e., the interfacial energy function has cusps and/or is non-convex). Various attempts have been made to extend phase-field models to strongly anisotropic (non-convex) interfacial energy [179-182]. Torabi el at. [183] reformulated the classical anisotropic energy formulation. The phase-field model can describe the growth of faceted interfaces. Phase-field models of dislocation dynamics [184, 185] use a strong anisotropic interfacial energy formulation which can describe a sharp interface with zero interfacial energy. These interfacial energy formulations could be important to describe the step and faceted interface in phase-field models of SLI evolution during crystal growth.

Nucleation processes play a critical role in the formation and evolution crystal microstructures, particularly in the context of step formation, growth direction selection, grain refinement, formation of precipitates, dislocations and twins. Nucleation is generally believed to be one of the most difficult processes to model. The formation of a critical nucleus is controlled by the energetic competition among a bulk free energy decrease which is proportional to the nucleus' volume, an interfacial energy increase which is proportional to interfacial area, and long range interaction energies such as elastic interaction etc. A critical nucleus is defined as a fluctuation of the composition or order parameter that results in the minimum free energy increase among all fluctuations leading to nucleation, i.e., the saddle point configuration along the minimum energy path (MEP) between the metastable initial phase represented by a local minimum in the free energy landscape and the equilibrium phase represented by the global minimum. The formation of a critical nucleus requires overcoming the minimum thermodynamic barrier. According to classical nucleation theory, the nucleation rate, which determines the particle density as well as the resultant microstructure, depends on the magnitude of the nucleation barrier, which usually changes with time and space. Therefore, a method should have the capability to correctly describe the energetic competition, nucleation barrier and nucleation rate in order to model the nucleation process. The phasefield approach can correctly describe the energetic competition during microstructure evolution. The morphology and concentration or order parameter profiles of a critical nucleus can be predicted. Homogeneous nucleation theory in unary and binary alloys was developed for the phase-field model [186]. Nucleation is incorporated into the simulations by including white noise into the governing equations of amplitude that forces nucleation in the spatial and time windows used or by introducing critical fluctuations based on the local composition. Phase-field models have been used to study the thermodynamic information of nucleus such as morphology, concentration profile and nucleation barrier of critical nucleus [187-189]. Based on classical nucleation theory, homogeneous nucleation process was integrated into phase-field simulations of phase separation in alloys [190]. Warren et al. extended the homogeneous nucleation theory to heterogeneous nucleation by introducing the wetting properties at interfaces into phase-field models [191].

It is believed that melt convection near an SLI strongly affects the interface morphology stability. Since solving the Navier-Stokes equations is time consuming because fluid incompressibility has to be ensured. The govern equations (fluid mass and momentum conserve equations) of fluids can also be described by Boltzmann equations [48-50]. Boltzmann equations use distribution functions to represent 
fluid behavior, and can be solved by lattice Boltzmann methods (LBM). The lattice Boltzmann method is presently a well-established tool to simulate fluid flows, especially flows in complex geometries. It can be easily modified to account for the thermal transport due to convection and diffusion, and buoyancy forces can also be incorporated without any problems. Other advantages of the method are its good stability and the ease of parallelization. Therefore, it seems natural to combine phase field model and lattice Boltzmann approaches for simulations of dendritic growth in external flows. Some attempts in this direction were made in Ref. [176-178]. A composite phase-field lattice-Boltzmann scheme is used to simulate dendritic growth from a supercooled melt, allowing for heat transport by both diffusion and convection. The phase-transition part of the problem is modeled by the phase-field approach of Karma and Rappel, whereas the flow of the liquid is computed by the lattice-Boltzmann-BGK (LBGK, referring to Bhatnagar, Gross, and Krook [192]) method into which interactions with solid and thermal convection are incorporated. Phase field models also take the effect of solute trapping and stresses on microstructure evolution into account [193-195].

From the survey of phase-field capabilities, we can see that all the important factors such as anisotropy of interfacial energy and mobility, solute trapping, temperature gradient, concentration gradient, melt convection, and defect nucleation, which may affect the interface morphological instability, can be taken into account in phase-field models. However, most phase-field simulations of SLI morphological stability focus on dendrite growth during solidification. For example, the effect of temperature gradient, solute trapping and growth rate on the interface morphology transition from flat to cellular were studied in Ni-Cu alloys during unidirectional dendrite growth by phase-field model [196]. In the simulations, temperature gradients of $2.5 \times 10^{4} \mathrm{~K} / \mathrm{cm}$ to $3.0 \times 10^{5} \mathrm{~K} / \mathrm{cm}$ and growth rates of $10^{-3} \mathrm{~cm} / \mathrm{s}$ to $3.5 \mathrm{~cm} / \mathrm{s}$ are considered. These growth conditions are few orders of magnitude higher than that (about $10^{-6} \mathrm{~cm} / \mathrm{s}$ ) during CZT crystal growth in typical vertical gradient freeze (Bridgman) growths [3]. In addition, the interface microstructures, which are important for interface morphological instability and defect generation during crystal growth, are often neglected during dendrite growth modeling.

Modeling SLI morphological instabilities during crystal growth is a big gap. How to extend existing mesoscale simulation capability to explore SLI morphological instabilities is a future research direction. One of challenges could be how to integrate step nucleation and growth into mesoscale models.

\subsubsection{Mesoscale modeling of defect generation during crystal growth}

Unidirectional crystal growth (Bridgman type growth) [91] is characterized by an imposed growth rate in a positive temperature gradient. The growth conditions are chosen such as to avoid nucleation of the product phase in the melt phase, to stabilize the SLI, to eliminate the defect nucleation and growth, and to obtain a macroscopically flat interface and high quality and large grain crystal. The ultimate goal of mesoscale modeling is to fundamentally understand the effect of growth conditions on the interface morphological stability of the SLI and defect generation during growth and annealing. During past four decades, there are intensive investigations of dendrite growth during solidification using phase field approaches and sharp interface methods [157]. The growth of unidirectional crystal growth structures, especially the interface morphological instability and defect generation during growth, remains comparatively less understood than that of dendrite growth. However, there are some starting simulations of the columnar growth of complex cellular/ dendritic array structure [197-199], faceted growth [200203], multi-grain growth [202, 204], precipitate evolution [205, 206], and twin formation [206] during unidirectional crystal growth by phase-field method, level set method and hybrid sharp interface and diffuse interface methods. 
Russo et al. developed a level set method for the motion of faceted interfaces in a polycrystal in three dimensions [202]. For given interface kinetics the model can capture the evolution of faceted interfaces as shown in Figure 10 [202]. The effect of adatom concentration and concentration fluctuations on the evolution of faceted interface was integrated into the level set model for modeling epitaxial growth kinetics [207]. One feature of this model is that steps are introduced by nucleation probability as a function of adatom density from kinetic Monte Carlo simulations. In addition, recent progress have been made to model the effect of melt convection, temperature and solute concentration on dendrite growth in pure materials and alloys using hybrid lattice Boltzmann, fixed-domain method, level set, and autoculaller methods [208-210].

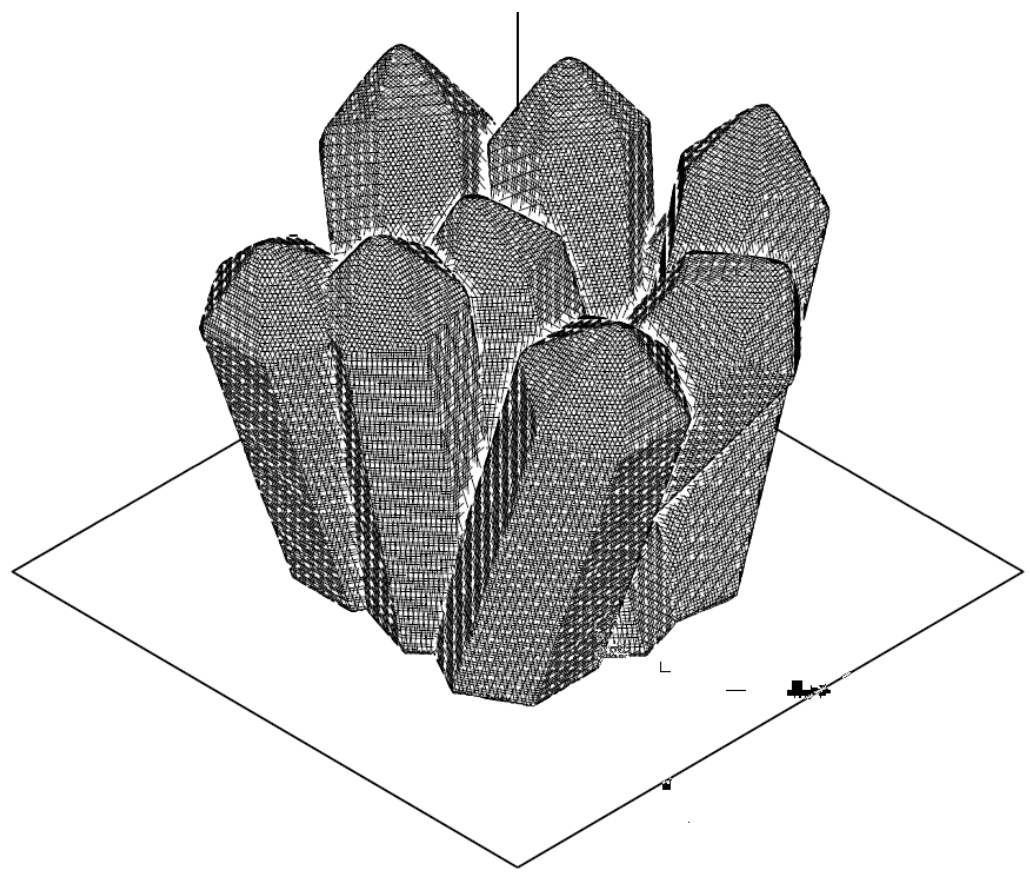

Figure 10. Evolution of nine needle-shaped crystals [202].

A phase field model with strongly anisotropic interfacial energy proposed by Torabi et al. [183] was successfully employed to simulate the faceted cellular growth in three dimensions [203]. Figure 11 shows the faceted interface evolution. Simulation results show that a linear relation of undercooling and growth velocity when the shape selection is completed at the late stage of the evolution but a nonlinear relation holds during the shape selection stage. During the facet cellular formation the crystal-melt interface is kept isothermal although the interface is sawtooth and areas with negative temperature gradient appear in the melts particularly at the ravine bottom of the sawtooth interface. It clearly demonstrates the capability of the phase field model in simulating the whole formation process of the faceted cellular and local temperature evolution. Phase-field models were also used to simulate the evolution of cellular structures in GeSi single crystal growth as a function of process parameters [199]; the growth competition of two grains with orientations (110) and (100) during directional solidification of silicon [204]; a step flow in epitaxial growth [211], and the effect of a coherent (111) twin plane on a $<110>$ twinned dendrite tip [205]. 


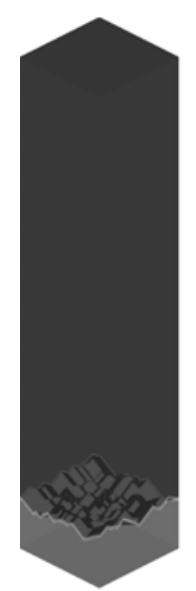

(a) $\mathrm{t}=5 \Delta \mathrm{t}_{0}$

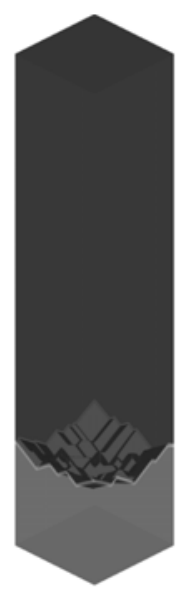

(b) $\mathrm{t}=10 \Delta \mathrm{t}_{0}$

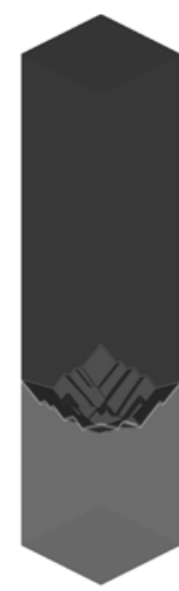

(c) $\mathrm{t}=15 \Delta \mathrm{t}_{0}$

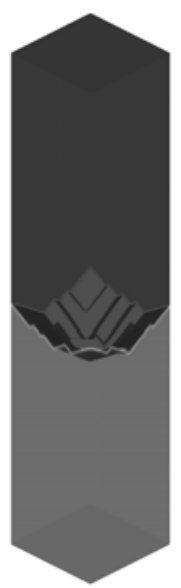

$(\mathrm{d}) \mathrm{t}=20 \Delta \mathrm{t}_{0}$

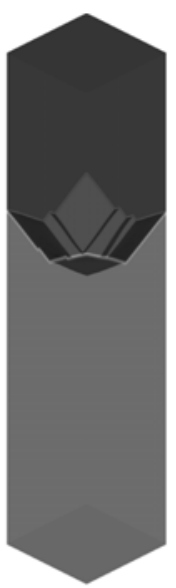

(e) $\mathrm{t}=25 \Delta \mathrm{t}_{0}$

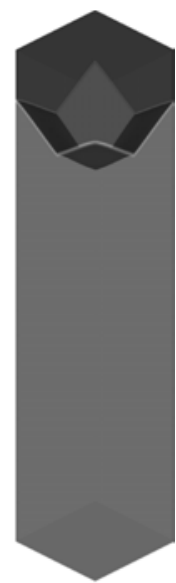

(f) $\mathrm{t}=30 \Delta \mathrm{t}_{0}$

Figure 11. Morphological evolution during the faceted cellular growth obtained from phase-field simulations [203].

Defects observed in growth crystals nucleate and grow not only at melt and crystal interface during growth but also during cooling and annealing process. Te precipitates are one of main defects that form during the cooling process of as-grown crystals and have profound effects on electronic properties of CdTe. Many factors such as the diffusion of intrinsic point defects (vacancy, interstitial, and antisites), internal stresses around the precipitates associated with the lattice mismatch between the precipitate and matrix, thermal stresses due to temperature gradients, extended defects (dislocations, twin and grain boundaries), non-stoichiometric composition, and the thermal treatment processing might all affect the formation and growth/dissolution of Te precipitates. A quantitative phase-field model for investigating the stability and evolution of a Te-precipitate in a Te-rich CdTe crystal during cooling and post heat treatment was developed [212]. The system is assumed to be three-phase (matrix CdTe, Te-precipitate, and void) equilibrium. With the thermodynamic and kinetic properties from experimental phase diagrams and thermodynamic calculations, the model enables one to study the effect of cooling rate, thermal stresses, and grain boundaries on Te-precipitate and void evolution kinetics. More recently, the thermal stress induced nucleation and growth of twins have been modeled by phase-field model [206]. The model assumes that twin nucleation, growth and de-twinning is a process of partial dislocation nucleation and slip on successive habit planes. Stacking fault energies, energy pathways (c surfaces), critical shear stresses for the formation of stacking faults and dislocation core energies are used to construct the thermodynamic model. There are more mature mesoscale mechanical methods to model dislocation evolution driven by internal and external stress fields [213-215].

\subsubsection{Dislocation network formation}

Existing models of dislocation formation in CdTe and other compound semiconductors suggest that these defects are easily formed during crystal growth [216] and that certain aspects of their production and geometry can be established with some precision [213-215, 217, 218]. However, the task of predicting dislocation structures formed during CdTe or CZT growth has not yet been achieved.

Discrete dislocation dynamics (DDD) is a mesoscale tool [219-228] that can be use for improved models of dislocations in CZT during solidification and crystal growth. Recent research [229] indicates that random dislocations can be assembled into a dislocation subgrain network using these methods, which is appealing for CZT growth simulations. DDD methods treat dislocations as discrete line defects 
with complete interaction forces and intersection results included in the computations. These methods are suitable for developing models of dislocation pattern formation in CdTe or CZT during crystal growth due to thermal stress fields and high-temperature annealing in the solid during growth. Essentially, by the time the growth has concluded and the CZT crystal has cooled the dislocation structures are expected to be fully at equilibrium, low-energy arrangements. Dislocation cells of about 100 to $1000 \mu \mathrm{m}$ are typical for as-grown VGF CZT crystals [216].

Importantly, DDD methods can be incorporated into phase field models and finite element models so that these important structural defects can be treated in a consistent crystal growth model [230-234]. One aspect of dislocations in CZT, which is a polar material in this regard, is that dislocations carry electrical charges depending upon the details of their structure [216]. Although this may have a larger effect on electron-hole transport in these materials, studies of dislocation structure, which may be needed for more detailed atomic models, and require an interatomic potential (IAP) able to account for charge transfer.

\subsubsection{Research directions of mesoscale methods}

Crystal growth occurs on time and length scales that span many orders of magnitude. The most basic physical processes such as step nucleation and solute diffusion occur on the atomic scale, i.e., on length scales of the order of $\AA$, and time scales that reflect the typical atomic vibration frequencies (i.e., $10^{-13} \mathrm{~s}$ ). On the other hand, a typical grain might be up to hundreds of microns in size, and its growth can take hours or days. Moreover, some of the phenomena that occur during crystal growth are inherently stochastic in nature. Thus, modeling crystal growth presents an enormous challenge to theoretical physicists and material scientists. It is almost impossible to seamlessly combine the different time and length scales as well as the necessary fluctuations into a single model. Different models with different length and time scales are required to model crystal growth process. Methods linking or delivering thermodynamic and kinetic knowledge from lower length scale to upper length scale are required to carry out the multiscale modeling of crystal growth. Mesoscale methods act as the bridge between atomistic and macro-scale modeling. The mesoscale simulations focus on the morphological evolution of SLI and grains, and the generation of different defects during crystal growth. The development of predictive mesoscale methods requires the progresses in the following directions:

1) Nucleation of steps, twins, dislocations and precipitates plays a central role in the evolution of SLI, grains, and defects. Existing mesoscale methods have very limited capability of handling the nucleation processes. The formation of a critical nucleus is controlled by the energetic competition among a bulk free energy decrease which is proportional to the nucleus' volume, an interfacial energy increase which is proportional to interfacial area, and long range interaction energies such as elastic interaction etc. In addition, the formation of a critical nucleus is a statistical process where random distribution of potential nucleation sites and fluctuations of local fields are important. The mesoscale methods have to take into account the energetic competition, fluctuations, and statistical processes for predicting the nucleation processes.

2) Strong anisotropic interface properties such energy and mobility of SLI, twins and grains, and long range interactions, including diffusion, heat transfer, melt convection, and elastic interaction are important thermodynamic and kinetic properties that govern the formation and evolution of microstructure and defects during crystal growth. Furthermore, the coupling among different defects and local fields is also important for predicting the microstructure and defect evolution. To integrate important physics into mesoscale models, the models require fine resolution to capture sharp 
interfaces and small nuclei as well as resolution to capture long range interactions among different fields. Different mesoscale models are needed to be developed for predicting the following:

a) SLI instability by considering step nucleation and lateral growth, solute trapping, and the effect of local temperature, concentration and melt convection.

b) Growth kinetics of unstable interfaces and Te inclusions, such as cellular and zigzag growth.

c) Formation of growth twins during unstable SLI growth.

d) Formation of deformation twins and dislocations during unstable SLI growth.

e) Precipitation during cooling.

f) Dislocation network evolution during cooling.

3) Mesoscale simulations of 3D microstructures and defect evolution are computational intensive processes. In addition, there are great challenges to solve the ill-posed microstructure evolution equations with sharp interfaces and possible large fluctuation in concentration, temperature, and convection fields. Therefore, efficient numerical methods are required to be developed.

4) Mesoscale simulations predict the evolution kinetics of SLI and different defects. The microstructure and evolution kinetics can be directly compared with the experimental observations. Specific experiments characterizing the formation and morphology of SLI and different defects are required for validating and improving the mesoscale models.

5) Quantitative mesoscale models are based on fundamental defect physics, defect energies, kinetic properties, and nucleation information at the SLI. Atomistic simulations of the following thermodynamic and kinetic properties are required for the quantitative mesoscale modeling:

a) Anisotropic interfacial energy, particularly for alloys and ordered compounds.

b) Anisotropic kinetic properties, particularly for step growth kinetics and solute trapping at ledges.

c) Nucleation mechanisms of steps, twins, dislocations, and precipitates.

d) Nucleation information of critical nuclei, such as energy barrier, critical size and morphology.

e) Effect of local fields on the thermodynamic and kinetic properties.

\subsection{Atomistic Methods and Interatomic Potential Considerations}

\subsubsection{Classical MD methods}

Because of large applications of molecular dynamics (MD) methods in materials science, a variety of techniques have been utilized over the years to develop reliable atomic potentials. The earliest classical potentials, mainly employed to study of liquids, only contained contributions from pair interactions. However, it was soon realized that pair potentials lack certain fundamental properties required for the accurate description of chemical and metallic bonding. One of the early successful attempts to include many-body effects was the introduction of the embedding functional [235], which depends on nonlinearly upon the coordination number of each atom. This development leads to the birth of the embedded atom method (EAM) [236, 237], which provides relatively accurate description of noble transition metals and their alloys, Tersoff potential formulism [238], which is based on the concept of bond order and has been applied to a large number of semiconductors, and Buckingham potential [239] or charge transfer potentials [240, 241] for oxides.

Atomistic simulations are increasingly being used as a tool to predict properties of materials in systems, such as nanomaterials, where direct measurement is time-consuming or extremely difficult. The success of atomistic simulations depends critically on the fidelity of a specific model of interatomic interactions to the area of application. Interatomic potentials in metallic systems are typically derived and 
optimized against a small number of validating experiments, and they necessarily work best for specific materials and phases. Thus, these potentials may accurately model one system and range of parameter space but be inappropriate for another. It is therefore essential that engineers and researchers know which interatomic potentials are available and how well they reproduce material properties of interest.

To build and disseminate a database of interatomic potentials, evaluations, and reference data, we began by soliciting feedback from researchers in industry, government, and academia to ascertain which properties are most relevant for industrial design processes. Such properties include, but are not limited to, molar volumes, phase stability, microstructural information, thermodynamics of interfaces and surfaces, diffusion, and melting temperatures. Additionally, it is important to know how these quantities change with decreasing size, especially as nanoscale materials become increasingly important. This feedback has been incorporated throughout the project, and is reflected in the activities described below.

\subsubsection{Embedded atom method and variants}

For materials like metals, a widely used semiempirical potential is the embedded-atom method (EAM), which incorporated an approximation to the many-atom interaction neglected by the pairpotential scheme [237, 242]. The EAM construction is based on the use of density functional theory, in which the energy of a collection of atoms can be expressed exactly by function of its electronic density. In EAM scheme, each atom is embedded in a host electron gas created by its neighboring atoms. The atomhost interaction is described using the embedding function incorporating some important many-atom interactions. It is possible to describe and understand interatomic interactions at defects in terms of either the embedding function or the effective many-atom interactions that arise from it. Over the last 20 years, a number of other many-body potential models have been proposed and developed with very similar approach, such as the Finish-Sinclair model that is based on second-order moment approximation of tightbinding [236, 243, 244], the glue model potential [245, 246] and the Sutton-Chen potentials [247]. As compared with pair potential, the EAM potential model overcomes several problems, such as the coordination independence of bond strength and the zero value for the Cauchy pressure for metals $\left(C_{12}-\right.$ $C_{44}=0$ ). Currently, EAM potentials have been applied to study many aspects of materials behavior in bcc, fcc and hep metals. In EAM, the total energy of a system can be described by

$$
\begin{gathered}
E=\frac{1}{2} \sum_{i} \sum_{j \neq i} \varphi_{i j}\left(r_{i j}\right)+\sum_{i} F\left[\rho_{i}\right] \\
\rho_{i}=\sum_{i \neq j} \rho^{a}\left(r_{i j}\right)
\end{gathered}
$$

where $\rho_{\mathrm{i}}$ is the electron density of the host atom at the site of atom $\mathrm{i}, \mathrm{F}\left[\rho_{\mathrm{i}}\right]$ is the embedding functional describing the energy to embed atom $i$ into the background electron density, and $\phi_{\mathrm{ij}}$ represents a pair potential between atoms $i$ and $j$. The popularity of the EAM model is due to its underpinnings in quantum mechanics and mathematical and computational simplicity, which allows modeling of a system consisting of several millions of atoms, with recent simulations involving more than $10^{9}$ atoms.

Most EAM potentials have been developed for metals and their alloys, but are generally fitted to limited number of experimental data and ab initio calculations. These fitted potential parameters may not be adequate to describe phase spaces where the potentials were not "trained," or far away from equilibrium states, thus exhibiting inevitable shortcomings. The EAM has been applied to a large variety of problems related to properties of metals, such as the structure, dynamics, phase transitions, vibrations, diffusion, and segregation. Some of the applications of the EAM appearing in the literature include bulk 
properties [248], grain boundaries [249], surfaces [250], alloys [251], defect properties [250, 252] and mechanical thermal dynamics properties [253]. In general, the potential parameters were optimized by adjusting the properties predicted by the potential to fit the experimental data, including the lattice parameters, elastic constants, cohesive energy and defect formation energies. In a recent application, the second-order elastic moduli and third-order moduli, as well as the cohesive energy and lattice constants, of a set of 12 cubic metals with fcc and bec structures were used as input to obtain the corresponding potential parameters for these metals $[254,255]$. The EAM is typically implemented in one of four different types of calculations: (a) energy minimization, (b) MD, (c) Monte Carlo (MC), or (d) vibrational-normal-mode analysis.

The lack of explicit three-body terms makes conventional EAM inappropriate for systems where directional bonding is important, such as semiconductors and elements from the middle of the transition series. However, there has been an application of these potentials to covalent materials, such as $\mathrm{Si}$, where a small modification of the mode was made to include these bond-bend forces [256]. The electron density of atom $\mathrm{i}$ includes more distant neighbors and angle-dependent density given by

$$
\rho_{i}=\sum_{i \neq j} \rho^{a}\left(r_{i j}\right)-a \sum_{\substack{j \neq i \\ k \neq i}}\left(1-3 \cos ^{2} \theta_{i j k}\right) \rho^{a}\left(r_{i j}\right) \rho^{a}\left(r_{i k}\right),
$$

where $\mathrm{r}_{\mathrm{ij}}$ is the distance between two atoms $\mathrm{i}$ and $\mathrm{j}, \theta_{\mathrm{ijk}}$ is the included angle between atoms $\mathrm{j}, \mathrm{i}$ and $\mathrm{k}$, and a is a constant to be determined by fitting to the share moduli. However, this potential has not been thoroughly tested and used. Recently, the angular dependence of interatomic interactions in the covalent materials is incorporated into the framework of the EAM potential, which is defined as Angulardependent EAM (A-EAM) potential $[257,258]$. It is believed that the A-EAM potential retains all the properties of the pure elements as predicted by the original SW, Tersoff, and EAM potentials, which provides an advantage in eliminating the need for extensive testing and limiting the scope of the potential parameterization to only the cross-interaction between the elements for composite materials. The A-EAM potentials have been employed to study an $\mathrm{Au}-\mathrm{Si}$ system, and the results are in good agreement with experimental data obtained for the enthalpy of mixing in the $\mathrm{Au}-\mathrm{Si}$ liquid alloy and the $\mathrm{Au}-\mathrm{Si}$ phase diagram. These initial investigations demonstrate the ability of the potential to provide an adequate description of the structural and thermodynamic properties of the alloy at different temperatures and in the whole range of compositions.

\subsubsection{Modified EAM (MEAM) potential and variants}

Although the EAM gives a more realistic picture of crystal properties than can be obtained by pair potentials, there are two assumptions. First, spherically averaged free atom densities represent the atomic electron densities, such that the potentials cannot accurately describe for the systems with a negative Cauchy discrepancy, particularly for hcp metals. Second, the host electron density is approximated by a linear superposition of the atomic densities of the constituents. These assumptions are too simple and cannot describe the actual situation well. Accordingly, Baskes modified the EAM to include directional bonding in the expression of electron density and applied it to silicon [256], a variety of cubic materials [259] and hcp materials [260], the so called MEAM potential. MEAM assumes that the energy per atom is a known function of the nearest neighbor distance in the reference structure for the element under consideration. An analytic form for the electron density at a given atom site arising from the other atoms and an analytic form for the embedding energy as a function of the electron density are also assumed. In this approach, the embedding function $F(\rho)$ can be expressed as 


$$
F(\rho)=A E_{c}\left(\rho / \rho_{e}\right) \ln \left(\rho / \rho_{e}\right)
$$

where $A$ is an adjustable parameter, $E_{c}$ is the cohesive energy, and $\rho_{e}$ is the electron density corresponding to the reference structure. The electron density at a given site is defined as the sum of term with $\mathrm{s}, \mathrm{p}, \mathrm{d}$, and f symmetry from the neighboring atoms. By including these angular terms in the background electron density, the angular forces can be introduced into the model and the electron density can written as

$$
\rho=\left[\sum_{h=0}^{3} t^{(h)}\left(\rho^{(h)}\right)^{2}\right]^{1 / 2}
$$

where $\mathrm{h}=0,3$ corresponds to $\mathrm{s}, \mathrm{p}, \mathrm{d}$ and $\mathrm{f}$ symmetry, respectively. For $\rho^{(\mathrm{h})}<<\rho^{(0)}$, the electron density can expressed using Tyler expansion. However, it is convenient to chose the values of $t(h)>0$, satisfying the positive electron density required by Eq. (5). An alternative expression that processes the appropriate asymptotic value for $\rho^{(\mathrm{h})}<<\rho^{(0)}, \mathrm{h}=1,3$ is given by

$$
\rho=\rho^{(0)} \exp \left(\frac{1}{2} \sum_{h=1}^{3} t^{(h)}\left(\rho^{(h)} / \rho^{(0)}\right)^{2}\right)
$$

The MEAM potential has been applied to many cubic metals, as well as $\mathrm{Si}$, including $\mathrm{Ge}, \mathrm{C}, \mathrm{H}, \mathrm{N}$ and O. In principle, the potential can be adopted for modeling of systems with mixed type of bonding, such as Mo-Si [261], Au-Si [262], and Au-Si-O [263]. In addition, several MEAM potentials have been suggested to study oxides and semiconductors, such as $\mathrm{SiO}_{2}$ [264], Alumina [265] and $\mathrm{SiC}$ [266], with charge transfer between the components explicitly incorporated into the models.

Similar to the MEAM scheme, several modifications to the potential form have been proposed. Pasianot et al. [267] added a new term in the form of total energy for the EAM to represent a many-body shear term related to bond angles in a global (average) sense. Zhang et al. [268] modified Johnson's analytic EAM, in a similar way to Pasianot. They added a modified analytic energy term $M(P)$ to the total energy expression for the EAM to express the difference between the actual total energy of a system of atoms and that calculated from the original EAM using a linear superposition of spherical atomic electron densities. The model was successful for calculating the vacancy diffusion mechanism for all bcc transition metals and the thermodynamic properties of their binary alloys. The second nearest-neighbor modified embedded-atom method (2NN EMEAM) formalism was proposed by Lee and Baskes [269], which has been used to describe a wide range of elements and their alloy systems and to explore many aspects of solid interfacial properties, especially the interfacial energy [270]. 


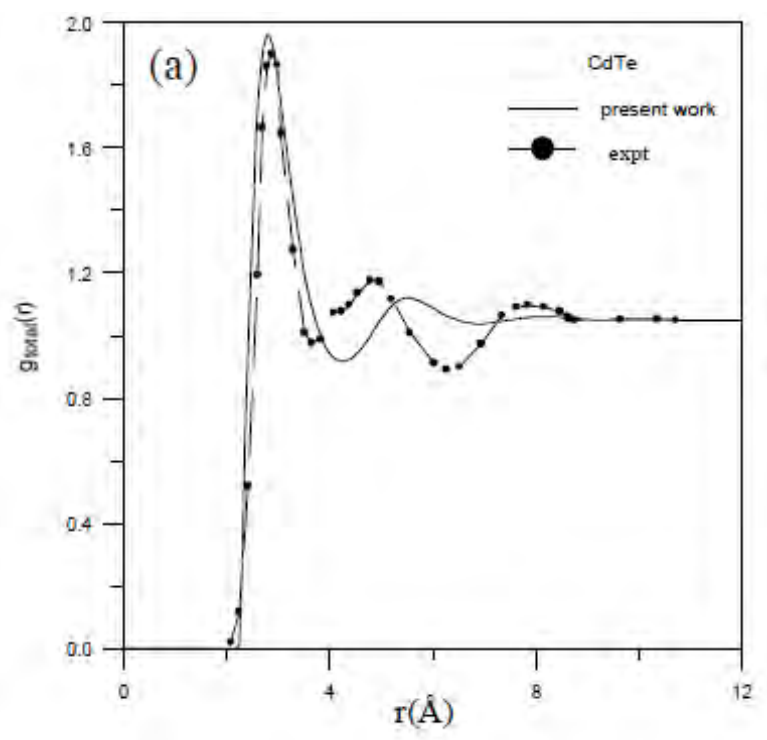

(a)

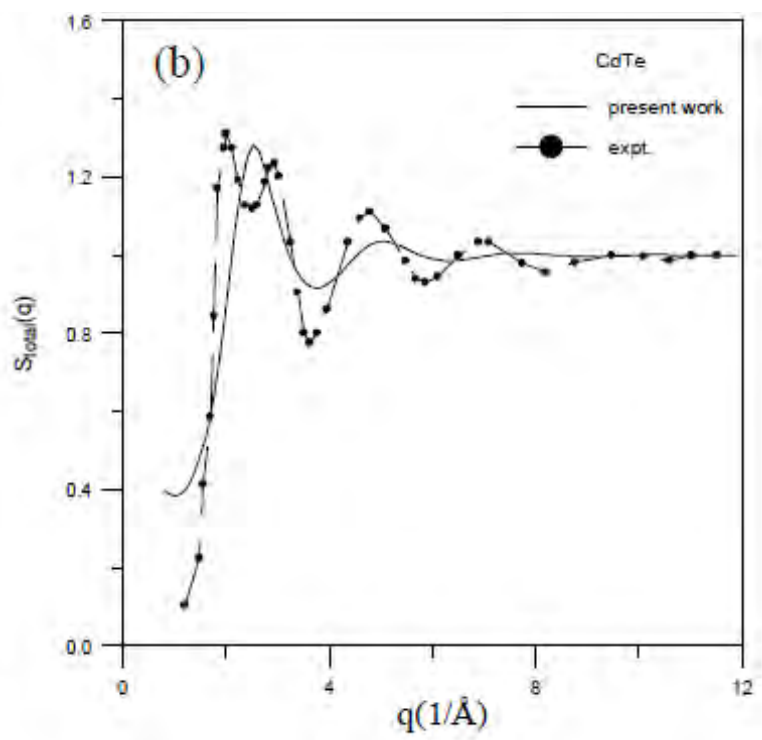

(b)

Figure 12. The calculated total pair correlation functions and structure factors using the AMEAM for liquid CdTe at $1375 \mathrm{~K}$ [271].

An analytic modified EAM potential (AMEAM) has been proposed for hcp metals [272] by adding one another energy modification term in the total energy expression. Two parameters $\alpha$ and $\beta$ that describe the anisotropy of hcp structure were added. The AMEAM potentials have been applied to calculate the thermodynamic properties of Mg-RE alloys [273], hcp rare-earth metals and their properties of various defects [274] and the liquid structure of hep chalcogenide metals and metal alloys [273, 274]. The calculated results have shown that these potentials reproduce exactly the observed $c / a$ ratio and all five elastic constants for each metal considered. Based on the AMEAM approach, Dalgic et al. [271] developed the potentials for $\mathrm{Cd}$, Te and $\mathrm{CdTe}$, which may give a good description only for their liquid. The effective interatomic interactions were constructed by a combination of the AMEAM potential functions with improving of the Finnis-Sinclair (FS) effective potential approximation based on the manbody approach [236] and used in their structural calculations at high temperatures. The static structure factors for pure metals and partial structure factors and pair distribution functions were calculated with one of the integral equation theory of liquids, namely variational modified hypernetted chain (VMHNC) theory which was successfully applied to metallic systems in the EAM calculations. The VMHNC results were compared with available experimental data, showing the reliability of the AMEAM for describing the structural properties of liquid $\mathrm{Cd}$, Te and CdTe systems. The calculated total pair correlation function, $g_{\text {total }}(r)$, and total structure factor, $S_{\text {total }}(q)$, for liquid CdTe alloy are plotted in Figures $12 \mathrm{a}$ and $12 \mathrm{~b}$, respectively, along with experimental data for comparison.

\subsubsection{Analytical Bond Potential}

The most popular potentials for covalently bonded systems include Stillinger-Weber (SW) potential [275, 276] for Si and Ge, Tersoff potential for Si and C [238], as well as Brenner potential [277] for hydrocarbon systems. The main advantage of the SW potential is its simplicity and fairly realistic description of crystalline silicon. The SW potentials are basically Lennard-Jones-type potentials and modified to include the short-range nature and the angle stiffness of the covalent bonding. The SW potentials consist of two-body and three-body terms. The two-body term is given by 


$$
V_{2}=\varepsilon A\left(B r_{i j}^{-4}-1\right) \exp \left\{\left(r_{i j}-a\right)^{-1}\right\} \text { and } r_{i j}=\frac{d_{i j}}{\sigma}
$$

where $d_{i j}$ is the bond length between atom $\mathrm{i}$ and atom $j$, and $\varepsilon$ the cohesive energy per bond. a is a dimensionless parameter which represents the cutoff distance of the interaction. The three-body term corresponds to the angle distortion energy and is given by

$$
V_{3}=\varepsilon \lambda \exp \left[\gamma\left(r_{i j}-a\right)^{-1}+\gamma\left(r_{i k}-a\right)^{-1}\right]\left(\cos \theta_{i j k}+\frac{1}{3}\right)^{2}
$$

where $\theta_{i j k}$ is the angle between bond $i j$ and $i k$.

The parameters in the above equations should be determined so that some basic properties of the material are reproduced from the potentials, including the cohesive energy, the equilibrium lattice constant, and the elastic properties. SW potential has gained big popularity and was used in numerous studies. The main advantage of this potential is its simplicity and fairly realistic description of crystalline silicon. The SW potentials have been used for the simulation of the crystal growth $[275,278]$ and the calculation of dislocation energy [279-281]. The critical thickness calculations using the SW potentials have also been reported for $\mathrm{Ge} / \mathrm{Si}[282,283]$. On the other hand, such atomistic simulations have seldom been attempted for III-V heterostructures. Khor et al. [284-287] proposed SW potentials for III-V compounds to simulate the critical thickness for InAs/GaAs, but the study of interatomic potentials for III-V materials is much less extensive than for $\mathrm{Si}$ and $\mathrm{Ge}$. The main advantage of this potential is its simplicity and fairly realistic description of crystalline silicon. However, it has certain serious limitations or transferability problems. The three-body term defines only one equilibrium configuration, which is difficult to extend it to an element such as carbon that has various bonding environments with equilibrium angles of $180^{\circ}, 120^{\circ}$, and $109.47^{\circ}$. Moreover, the build-in tetrahedral angle causes problem in many situations, such as the too low coordination in liquid, incorrect surface structures (surface relaxation cannot be reproduced) and incorrect energy and structure of small clusters.

More realistic, so-called bond-order potentials (Tersoff type) have been developed for $\mathrm{Si}, \mathrm{Ge}, \mathrm{C}$ and covalently bonded semiconductors, such as $\mathrm{SiC}, \mathrm{GaAs}$ and $\mathrm{GaN}$. The construction of Tersoff potentials is based on the formalism of analytic bond order potentials, initially proposed by Abell [288]. The central idea for this type of potential is that the binding energy of atoms is calculated using pairwise nearest neighbor interactions that are, however, modified by the local environment with many-body approximation. Tersoff employed this idea to develop the bond-order potential for Si [289], C [290], Si-C [291], Ge and Si-Ge [291] structures. In Tersoff's model, the interaction between atoms i and j, $\mathrm{V}_{\mathrm{ij}}$, can be described by

$$
V_{i j}=f_{c}\left(r_{i j}\right)\left[V^{R}\left(r_{i j}\right)+b_{i j} V^{A}\left(r_{i j}\right)\right]
$$

The function $V^{R}\left(r_{i j}\right)$ represents the repulsive pairwise potential, while the $V^{A}(r i j)$ describes the attractive bonding due to the valence electrons. The three-body term $\left(b_{i j}\right)$, which moderates the attractive term, plays the role of the bond order, which is used to capture the effect on the strength of the bonds. The form of the bond-order term was chosen such that the energy per bond is a monotonically decreasing function of coordination. A short-ranged cutoff $f_{c}\left(r_{i j}\right)$ is used so that the forces smoothly go to zero at the first neighbor shell of the structure of interest. The repulsive and attractive terms are given by 


$$
V^{R}\left(r_{i j}\right)=A_{i j} \exp \left(-\lambda_{i j} r_{i j}\right) \text { and } V^{A}\left(r_{i j}\right)=-B_{i j} \exp \left(-\mu_{i j} r_{i j}\right)
$$

However, other formulas are also used, such as Morse potentials, to describe the repulsive and attractive interactions [292]. The cut-off function, $f_{c}\left(r_{i j}\right)$, is used to restrict the pair interaction to the nearest neighbors and is defined as:

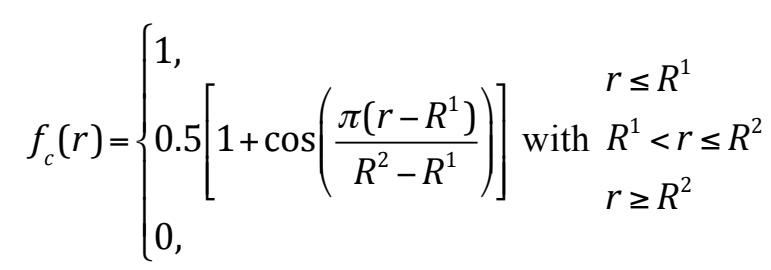

where $R^{l}$ and $R^{2}$ are adjustable parameters. It should be noticed that the second derivative of the cut-off function is discontinuous, and thus, the low-order algorithms should be used in the dynamics simulations. The three-body term, $b_{i j}$, is given by

$$
b_{i j}=\chi_{i j}\left[1+\beta_{i}^{n i} \xi_{i j}^{n i}\right]^{-0.5 n i} \text { and } \xi_{i j}=\sum_{k \neq i, j} f_{c}\left(r_{i k}\right) \omega_{i k} g\left(\theta_{i j k}\right)
$$

where the angular function $g(\theta)$ is described by

$$
g\left(\theta_{i j k}\right)=1+\frac{c_{i}^{2}}{d_{i}^{2}}-\frac{c_{i}^{2}}{h_{i} \cos \left(\theta_{i j k}\right)^{2}}
$$

where the labels $i, j$ and $k$ refer to the atoms with $i j k$ bonds and $r_{i j}$ and $r_{i k}$ represent the lengths of the $i j$ and $i k$ bonds whose angle is $\theta_{i j k}$. The Tersoff potential works in a broader spectrum of situations than the Stillinger-Weber potential, however it is not exempt from problems. One of the biggest problems is perhaps that the fitting process is difficult: with six functions to fit and angular terms, finding a good parameterization is not an easy task.

\subsubsection{Outlook for CdTe and CZT}

In terms of CdTe, there are two many-body interatomic potentials for CdTe that are already developed, one with the Stillinger-Weber scheme [293], and the other with a Tersoff approach that was slightly modified for a potential [294] by Wang and Rockett. The SW potential for Cd-Te the interaction was fitted to bulk atomization energies, lattice constants, and melting temperatures, while the Cd-Cd and Te-Te potentials were determined by considering these elements arranged in a fictitious diamond structure with the same nearest neighbor separation as the actual elements. The authors argued that this artificial expedient seems necessary to construct $\mathrm{Cd}-\mathrm{Cd}$ and $\mathrm{Te}-\mathrm{Te}$ potential appropriate for use in a zincblende -structure alloy. The potentials were used to the liquid-vapor interface of CdTe by a Monte Carlo technique. The calculated heat of fusion and elastic constants agree well with experiments. The calculated surface tension is found to be about $220 \mathrm{ergs} / \mathrm{cm}^{2}$, in good agreement with experimental estimates. The surface region is found to be $\mathrm{Cd}$ rich, even though elemental $\mathrm{Cd}$ has a higher surface tension than elemental Te. Also, these potentials were employed to determine the melting temperature, the heat of fusion at melting, and on the relative phase densities with a particular emphasis on the melting line [295]. The results demonstrated that a liquid with a density slightly less than that of the solid and, hence, the pressure-temperature melting line has a positive slope. The pair-correlation structure of the liquid was determined and favorably compared to neutron-scattering data and to ab initio simulations. 
The liquid-solid interface is discussed using density profiles and a short-range order parameter for models having principal orientations along $<100>,<110>$, and $<111>$ crystallographic directions.

More recently, Ward et al. [296] assessed the applicability of two potentials for MD studies of defect interaction and defect formation under thermomechanical conditions and identified a method to improve these potential models, namely the Stillinger-Webber (SW) format [275], and the Rockett modification the Tersoff potential (TR) [294]. A variety of elemental and compound lattices, defects, and surfaces performed using the two potentials were compared with those from experiments and/or $a b$ initio calculations. The suitability of the potentials for disturbed configurations is also tested using melting temperature calculations and vapor deposition simulations. It was found that the existing SW and TR parameterizations of the CdTe potential both give incorrect lowest energy elemental $\mathrm{Cd}$ and Te structures and thus, cannot be used for $\mathrm{Cd}$ or Te simulations. Also, both parameterizations do not capture the lowest energy surface reconstructions of the zincblende CdTe compound. It is unlikely that the potential can be used to study structure evolution, defect formation, and defect interaction processes even for zincblende CdTe. The SW CdTe potential correctly reproduces the melting temperature, the lowest energy, and the crystalline growth for the ground state zincblende structure of CdTe, which suggests that SW potential can be used to study CdTe for specific properties. On the other hand, the TR CdTe potential predicts a wrong melting temperature and an amorphous growth of the zincblende $\mathrm{CdTe}$, and a wrong $\mathrm{B} 2(\mathrm{CsCl})$ structure for CdTe. The Tersoff-Rockett parameterization, therefore, cannot be used for CdTe simulations. The Stillinger-Weber parameterization has reasonable elastic properties for open structures like zincblende CdTe, but significantly over predicts the elastic constants for closely packed structures. Consequently, simulations to study mechanical properties during high pressure and high density should be avoided with the SW parameterization. However, Ward et al. [296] suggested a two-step parameterization to improve the transferability of the TR potential beyond that of the SW potential. Further, full bond-order potentials were proposed to resolve many of the issues associated with SW and TR potentials [297, 298]. The model requires further testing for crystal growth simulations.

We have begun testing a variety of CdTe potentials and some preliminary results are shown in Figure 13 where we plot $g(r)$ data from each of four potentials from equilibrated liquid models held at $1365 \mathrm{~K}$ for several picoseconds. The four potentials are VASP DFT code [299, 300], SW [293], a BOP [298], and a modified-SW potential developed at PNNL. The total $g(r)$ data and partial $g(r)$ data are shown. Table 1 then lists the coordination numbers $\left(C_{i k}\right)$ computed from the total and partial $g(r)$ data using an $R_{\min }$ value of $0.365 \mathrm{~nm}$. The $\mathrm{CDN}$, or compositional disorder number, calculated from $\left(C_{C d C d}+C_{T e T e}\right) /\left(2 C_{C d T e}\right)$ indicates the degree to which the liquid is close to a random mixture, where $\mathrm{CDN}=1$, or to a zincblende solid, where $\mathrm{CDN}=0$. The $\mathrm{CDN}$ values for these potentials indicates that $\mathrm{CdTe}$ is a structured liquid at $1365 \mathrm{~K}$ with a nearly tetrahedral coordination but with undercoordinated Te as expected. These are some of the measures that can be used to compare interatomic potentials for CdTe liquid structure. Other measures include Te-Te chain formation and various bond angle distributions.

Table 1. Coordination Number Data for CdTe Liquid at $1365 \mathrm{~K}$ for Four Potentials

\begin{tabular}{|l|l|l|l|l|}
\hline $\mathbf{C}_{\text {ik }}$ & VASP & SW & BOP & PNNL \\
\hline Total & 3.50 & 3.48 & 3.60 & 3.50 \\
\hline CdTe & 2.63 & 2.43 & 2.54 & 2.50 \\
\hline CdCd & 1.12 & 0.83 & 1.10 & 0.86 \\
\hline TeTe & 0.62 & 1.25 & 1.01 & 0.96 \\
\hline CDN & 0.33 & 0.43 & 0.42 & 0.36 \\
\hline
\end{tabular}




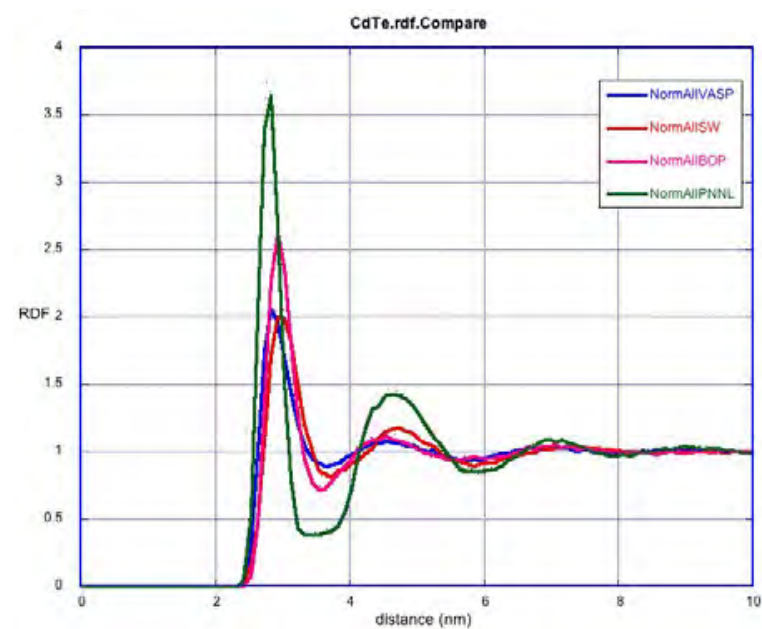

(a)

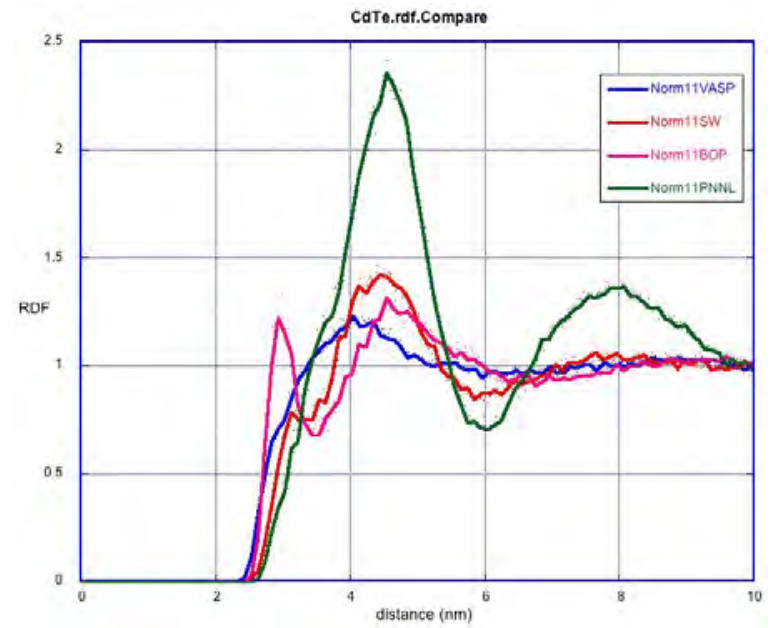

(c)

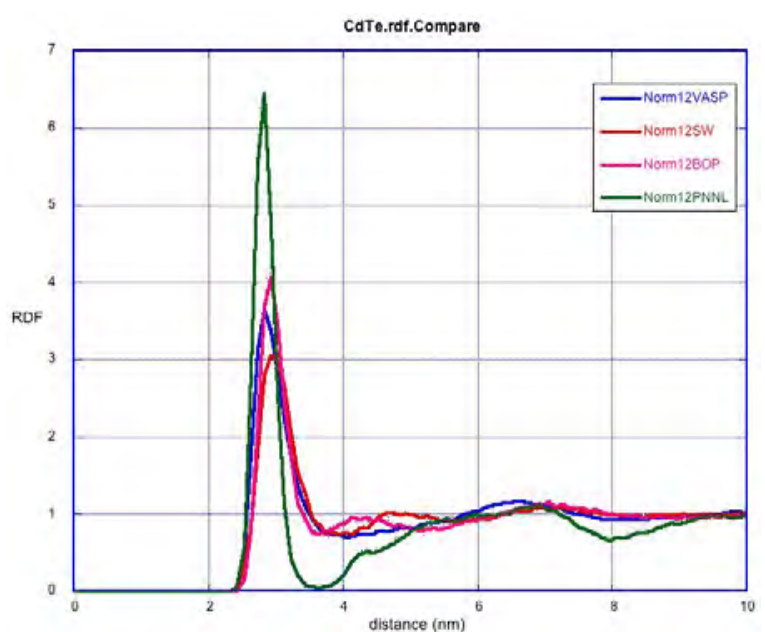

(b)

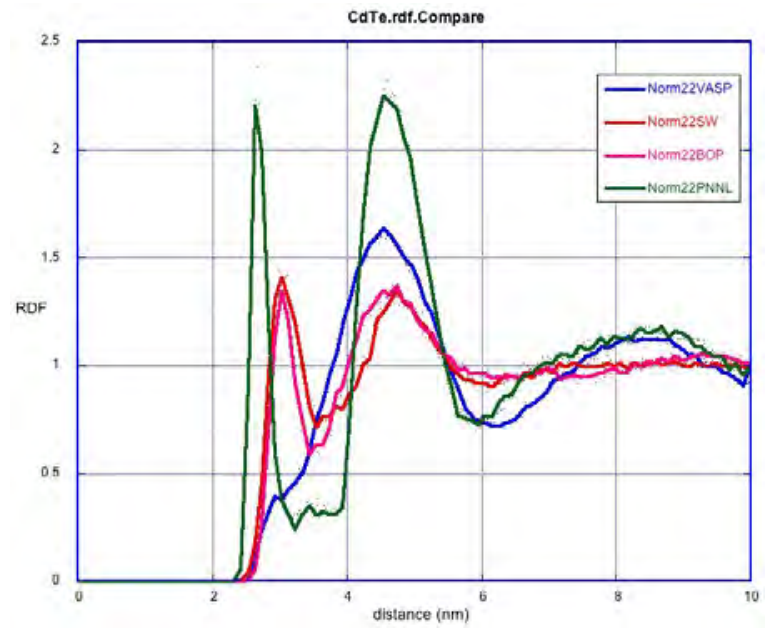

(d)

Figure 13. Total and partial pair correlation data from four different CdTe potentials for an equilibrated CdTe liquid model at $1365 \mathrm{~K}$. Shown in (a) is the total $\mathrm{g}(\mathrm{r})$, in (b) the CdTe partial, in (c) the CdCd partial, and in (d) the TeTe partial. This data was used to compute local coordination numbers for each and a compositional disorder number, $\mathrm{CDN}$ for each.

\subsection{Kinetic Monte Carlo methods}

Kinetic Monte Carlo (KMC) simulations and phase field models will enable to extend ab initio MD (AIMD) and MD simulations to the mesoscale. The key challenge in extending the time scale is to simulate a sufficient number of very fast events with small time steps (e.g. atomic diffusion jumps of interstitials) to reach steady-state, while still simulating the infrequent events with larger time steps (e.g. trapping and de-trapping of defects, defect annihilation, coalescence of small clusters and nucleation of second phase). The infrequent events are important, because they control nucleation and growth of second phase or precipitate and the overall microstructural evolution. In general, KMC simulations assume a lattice crystal and the atoms can move through lattice sites that are the elementary processes in a model system. To model the deposition and the diffusion on a surface or in a crystal, the probability of a diffusion process should be determined, which can be described by an Arrhenius equation, 


$$
\mathrm{p}=v_{0} \exp \left(-E_{\mathrm{D}} / k_{\mathrm{B}} T\right)
$$

where $v_{0}$ is the attempt frequency, $k_{B}$ is Boltzmann's constant, and $E_{D}$ is the diffusion barrier which differs for different processes. The timesteps in the KMC algorithm are obtained by summing over all possible processes

$$
\Delta \mathrm{t}=\frac{1}{\sum_{v \neq \mu} \mathrm{P}(v \rightarrow \mu)}
$$

where $P(\mu \rightarrow v)$ denotes the hopping probability from site $\mu$ to site $v$ according to (14). Diffusion processes to any one of the nearest-neighbor sites are considered.

The main steps in a KMC algorithm are outlined in Figure 14. The underlying principle in all KMC algorithms is the random selection of a process based on the transition probabilities of all processes, execution of the selected process (i.e., appropriately modifying the configuration of the system), and updating the time clock and the transition probabilities. In mathematical terms, the KMC method follows a discrete Markov evolution of the system with continuous time increments given by an exponential distribution. Each of the steps in the entire flowchart presents specific challenges. Subtle differences between different KMC algorithms originate from (1) the selection of a process and (2) determining the time increment after a process is executed. However, KLMC simulations require information on the migration energies of defects and entropies, binding energies, and interaction energies, as well as the initial distribution of defects produced and the initial microstructural features of the material. In KMC simulations, the underlying microstructural structure is decorated with mobile and immobile lattice defects (such as interstitials, vacancies and their clusters), and the simulations follow the migration and

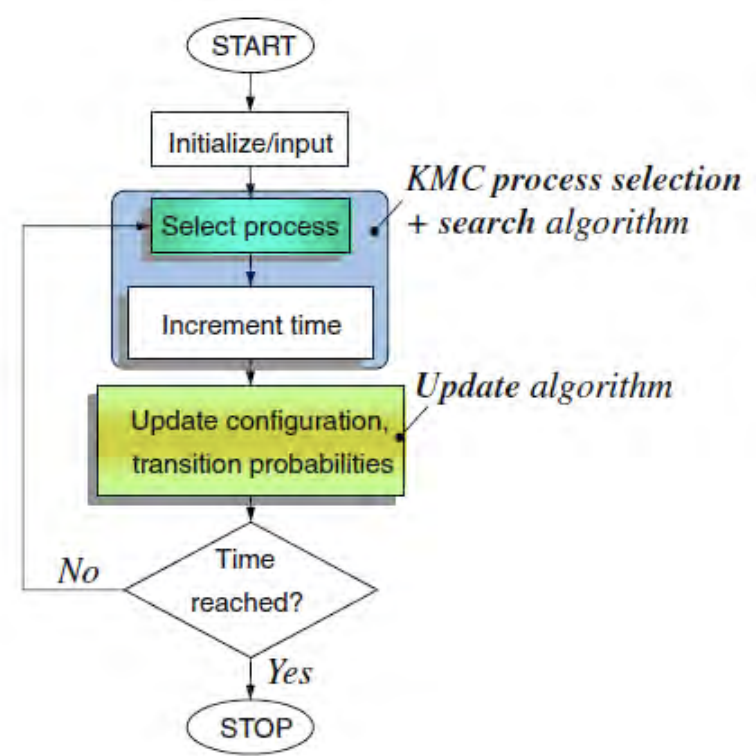

Figure 14. Flowchart showing for a KMC algorithm, where shaded boxes denote computationally most expensive steps. interaction of these defects on the crystal lattice and at interfaces. With various defect concentrations, the KLMC simulations can be used to determine the types of interfaces that will avoid defect accumulation and chemical segregation and those that promote phase transitions. In general, the necessary input parameters for KMC simulation can be obtained from the database of DFT and classical MD simulation results. The KMC results, which will also populate the database, can be compared directly to experimental data. Also, these simulation results on the initial stages of interface structures between liquid and solid, and free energies of interfaces with different defect concentrations will serve as inputs for the phase field model. KMC simulation is an efficient and established tool to obtain information about the statistical behavior of crystal growth on an atomic scale. 
Previously, KMC algorithms are used for understanding growth kinetics in epitaxial systems [301, 302]. A KMC model for the formation of liquid droplets, including an energy parameter simulating the surface tension, and a model for crystallite growth inside these droplets, taking into account the solute diffusion, has been developed [303]. The KMC models were employed to simulate the deposition of indium droplets on a glass surface and the subsequent formation of silicon microcrystals inside these droplets, as well as the influence of growth temperature, flux of incoming particles, surface coverage, and in particular an energy parameter simulating the surface tension, upon the morphology of growth. The simulations explain the formation of silicon crystal structures in good agreement with the experiment. The dependence of their shape and the conditions of formation on the growth parameters is investigated in detail. However, to obtain an understanding of the thermodynamics of liquid droplet formation on a substrate, the free enthalpy (Gibbs free energy), which describes the nucleation energy, needs to be determined. The free enthalpy can be obtained using MD simulations, with accurate interatomic potentials. Figure 15 shows an example of the formation of liquid droplets of indium on a molybdenum surface from the vapor phase, and the subsequent growth of silicon crystallites inside these droplets by a vapor-liquid-solid mechanism [303, 304].
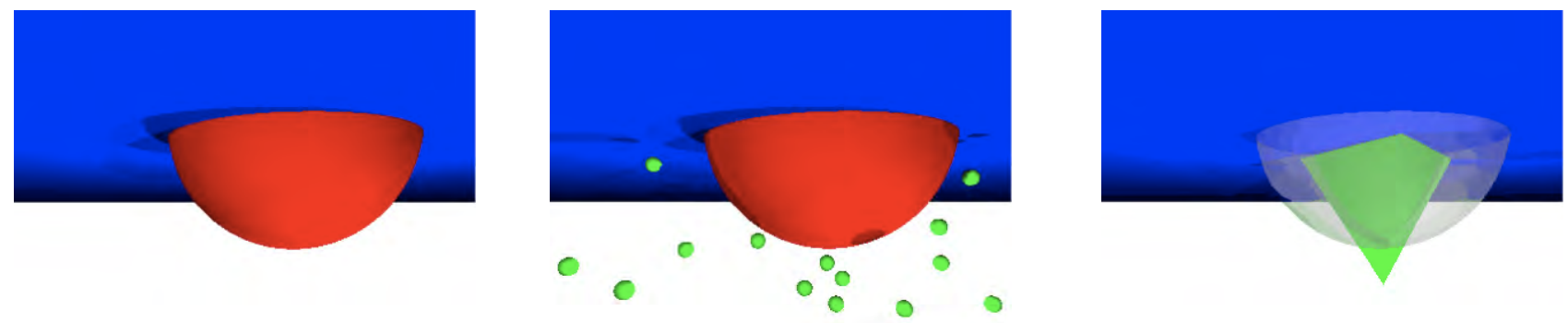

Figure 15. The main steps of growth: (a) Indium droplet (red) on a molybdenum substrate surface (blue), (b) vapor deposition of silicon atoms (green) and diffusion into the droplet and (c) crystallized silicon inside an indium droplet [303].

The formation of facets during solid phase epitaxy regrowth (SPER) in Si has been explored using a lattice kinetic Monte Carlo approach [305]. In this model, the silicon lattice in the crystalline regions is introduced and assumes that the recrystallization rate dependence for different orientations on the quality of the available crystalline template is assumed. In particular, each atom in the amorphous phase needs to form two undistorted bonds with the crystal. This happens naturally for (100) oriented surfaces. For (110) surfaces, a cluster of two adjacent atoms in the amorphous phase is necessary for each atom to complete two undistorted bonds, and a cluster of three atoms is necessary for (111) surfaces. This is modeled using three different prefactors to simulate the different frequencies at which atoms in the amorphous phase join the crystalline one. Therefore, the lattice atoms

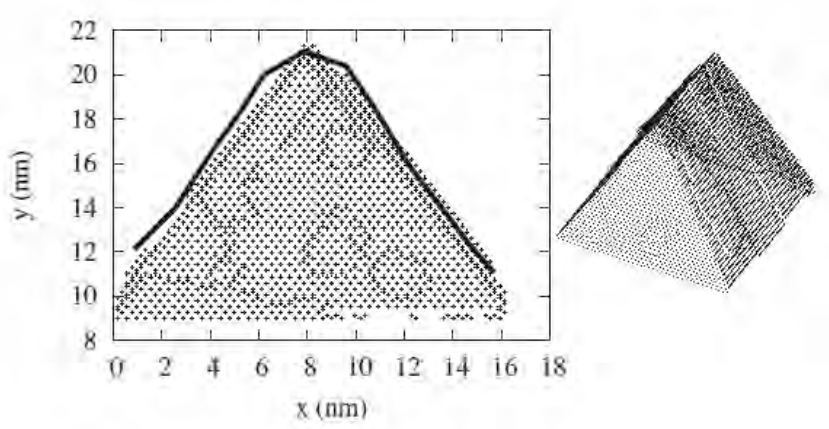

Figure 16. Left: arrow tip formation during SPER of a thin fin $\left(16 \times 50 \mathrm{~nm}^{2}\right)$. Simulation results (symbols) and the experimental amorphous/crystalline regrowth (line) after a $60 \mathrm{~s}, 600^{\circ} \mathrm{C}$ annealing. Right: $3 \mathrm{D}$ view of the same simulation. 
produce different crystalline planes growing with different planar velocities. In particular, the model explains the arrow tip formation during SPER of thin silicon fins typical for fin field effect transistors and the formation of trenches in rectangular-shaped amorphized regions in (001) and (011) silicon, caused by the distortion of the lattice by shear strain and propagated by (111) facets. Figure 16 compares the experimental amorphous/crystalline interface in thin-body silicon for a (100) substrate pattern in the (110) direction and surrounded by silicon oxide with the simulation results. After an implant and a $60 \mathrm{~s}$ annealing at $873 \mathrm{~K}$, the regrowth is incomplete but the facets are already formed following (111) planes. Since the silicon is surrounded by $\mathrm{Si} / \mathrm{SiO}_{2}$ interfaces, and these interfaces do not provide a suitable template for recrystallization, it regrows faster in the middle than at the sides. This physical effect is captured in the model without extra assumptions. Once the planes begin to form, they are pretty stable because recrystallization on (111) planes is very slow.

Chopra et al. [306] developed an order-parameter-based Monte Carlo simulation of crystallization and phase transitions. The method relies on a random walk in order parameter $\phi\left(q^{N}\right)$ space to calculate a free energy profile between the two coexisting phases. The energy and volume data generated over the course of the simulation are subsequently reweighed to identify the precise conditions for phase coexistence. The usefulness of the method is demonstrated in the context of crystallization of a purely repulsive LennardJones system. A systematic analysis of precritical and critical nuclei as a function of supercooling reveals a gradual change from a bcc to an fcc structure inside the crystalline nucleus as it grows at large degrees of supercooling. The method is generally applicable and is expected to find applications in systems for which two or more coexisting phases can be distinguished through one or more order parameters.

More recently, MC method has been developed to study stress control in polycrystalline thin films [307]. The diffusion of adatoms into grain boundaries (GBs) of polycrystalline thin film during vapor deposition affects the stress that develops and the film's subsequent performance. Based on polycrystalline kinetic Monte Carlo simulations of $\mathrm{Cu}$ (111) thin films with In surfactant, a new mechanism of modifying the stress by controlling adatom diffusion into GBs through the use of surfactants is proposed. Figure 17 shows the atomic configurations of thin film surface, where there are about 110000 sites in each surface layer, and the deposition ends with a coverage of 0.2 ML (or 22000 atoms). Without surfactant, the islands are large and their density is low. The random introduction of surfactant (or replacement of $\mathrm{Cu}$ by $\mathrm{In}$ ) at $8 \%$ concentration on the top substrate layer leads to reduced islands size and higher island density. However, the study demonstrates that the surfactant slows down adatom diffusion and increases its concentration. As a result, the product of adatom diffusivity and concentration decreases as surfactant concentration increases. This product is proportional to the adatom flux to GBs, and its decrease leads to smaller number of disappeared vacancies in the GBs and thereby the reduction in compressive stress. It should be noted that the

(a)
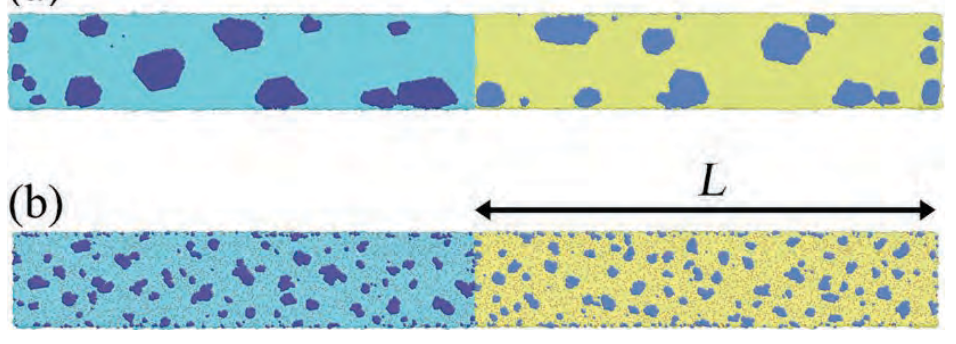

Figure 17. Top view of the surface of a bicrystal substrate and 0.2 ML thin film (in the form of islands), without (a) and with (b) surfactants of $8 \%$ in concentration. Grain size $\mathrm{L}=125 \mathrm{~nm}$ and temperature $\mathrm{T}=300 \mathrm{~K}$. binding energies of atoms to GBs, migration energies of adatoms and interactions between $\mathrm{Cu}$ and $\mathrm{In}$ atoms are determined buy either ab initio calculations or molecular dynamics simulations with empirical interatomic potentials, which may present challenging for MC simulations to cover all possible transition 
states over large potential surfaces and complex microstructures.

\subsection{Ab Initio Methods and Approaches}

\subsubsection{Large Scale Electronic Structure Calculations}

The need to design high performance materials with properties tuned to perform targeted and more efficient processes will require a much larger dependence on molecular simulation for interpretation, impact assessment and design. While there is significant averaging involved in reducing the dimensionality and coarsening time resolution in these problems, the specificity of the atomic level chemistry is evident and essential to predicting the behavior of the system at the larger scales. The computational challenge of simulating such systems is to predict their behavior for very large scales while retaining information from the atomic level. The well-developed tools of quantum chemistry and physics have been shown to approach the accuracy required. However, currently there are only a few programs (e.g. NWChem) that can simulate up to a few thousand atoms at this level of accuracy. While these developments are impressive, these systems sizes are not large enough to simulate many important material systems such as grain boundaries, dislocations, cracks, nanoparticle inclusions, as well as nanoparticles, defected mineral surfaces. New methods, which can scale up current electronic structure calculation by 10 - to 100 -fold are needed.

Although Density Functional Theory (DFT) methods and ab initio methods such as Møller-Plesset theory [308] are adequate to model complex materials systems, there are still significant limitations to the general application of these methods. DFT calculations require the self-consistent solution to $N$ coupled three-dimensional (3D) nonlinear eigenvalue equations, where $N$ is the number of electrons in the system. The solution to this problem requires expansion of the electron orbital in basis functions. The most common basis functions are either local atom centered functions or plane-waves (with pseudopotentials). These techniques produce useful results but both these methods suffer from the use of basis functions that do not have compact support, resulting in dense matrix operators that cannot be effectively parallelized beyond a certain size (i.e. for a basis of size $N_{g}$,

$N_{g}$ cpus can be used but the cost grows at $N_{g}{ }^{3}$ ) and computationally intensive transforms which are difficult to implement efficiently on emerging petascale computers (Figure 18). In fact very few DFT and $a b$ initio programs are known to scale beyond 1000 cpus. These fundamental limits to parallelization will result in severe restrictions to the size of the systems that will be able to be treated effectively in the near future. These problems are made even more difficult for applications that require numerous evaluations of the system energy, either to treat motion or to search for complex equilibrium structures.

At present the scaling up of current DFT algorithms is primarily hampered by their use dense matrix operations which scale as $N^{3}$.

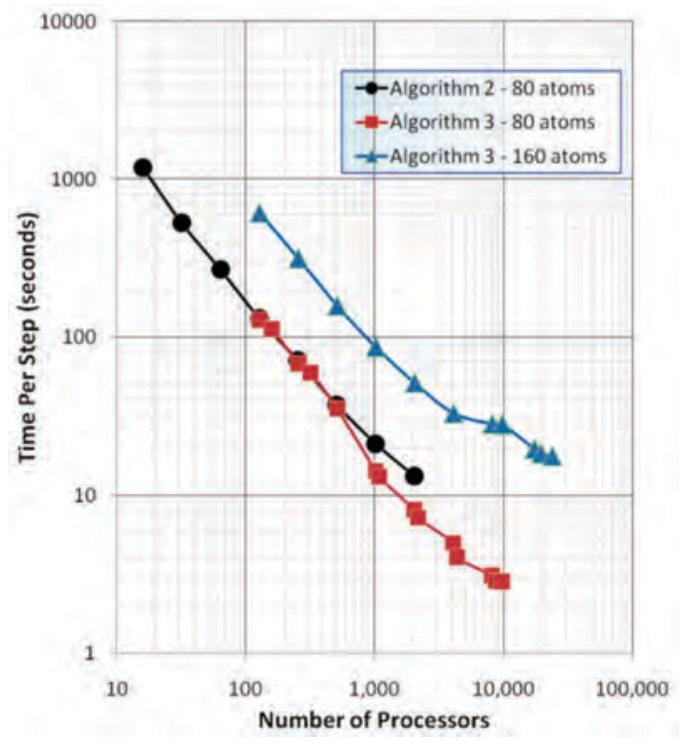

Figure 18. Parallel timings for Hybrid DFT of ironoxides 
These dense matrix operations are the result of these algorithms explicitly maintaining orthonormality between the $N$ Kohn-Sham orbitals. Several approaches for maintaining orthonormality, which scale as $N^{2}$, have been proposed [309]. In general, these algorithms use an inverse iteration (or Green's function) strategy to define a subspace over an energy range that is then subsequently diagonalized.

Even though a couple of groups [310-314] have implemented plane-wave methods that scale from 1000-10,000's of cpus (and even larger cpus for hybrid DFT) by using 2D parallel data decompositions over the number of electrons and plane-wave expansion alongside non-trivial efforts to mask latency. Ultimately, however, the scalability of these methods is limited by the scalability of the FFT algorithm, which is limited to $O\left(N_{g}{ }^{l / 3}\right.$ ) processor (or processor groups), where $N_{g}$ is the number of FFT grid points. The limitations of these standard solvers have motivated the development of various finite element method (FEM) and other real space solvers to the DFT and $a b$ initio eigenvalue equations [2]. With the FEM method the quantum mechanical operators can be represented by $O\left(N_{g}\right)$ sparse matrices, which can in principle limit the communications per processor to be $O(1)$, making them excellent candidates for the next generation of solvers on emerging petascale computers. In addition adaptive FEM methods have been shown to be able to describe the multiple length scales present in the exact singular electron-ion potential by telescoping down to the singularity [315]. Despite the fact that FEM methods have been successfully applied to complex systems of modest size, present methods of solution have not been widely accepted on the grounds of accuracy and efficiency especially for a dynamical calculation. However, recent work using real space solvers by Norskov [316], Fattebert [317], Pask [318], Bylaska [315], and Harrison [319] and Goedecker [320] and others have shown promise.

Given the advances in real space methods it seems sensible to pursue the development of new $O(N)$ electronic structure approaches targeted towards large scale material systems. These methods are still in their infancy and a considerable amount of work will be to be done to make these methods feasible for large-scale CZT simulations. The essence of $O(N)$ approaches is to eliminate the need for the costly $O\left(N^{2}-\right.$ $N^{3}$ ) orthogonalizations by using a sparse a linear representation of the electronic structure problem. Currently, there are only a couple of programs which implement $O(N)$ approaches. The most well known is the Siesta program. The approach used in Siesta is based on representing the $N$ molecular orbitals as localized orbitals. Unfortunately, this approach has been plagued by several problems that have limited its use to expert users, including systems that can have multiple ground state solutions and the method's sensitivity to the choice of localized orbitals. Further development may improve these types of localized orbital methods. Another approach that seems promising is the density matrix formulation. In this method, the solution to the non-linear electronic structure problem is represented using a density matrix instead of $N$ molecular orbitals. With this type of approach large numbers of atoms can be treated on single cpus. Equally important, it can be combined with sparse iterative methods that contain a high degree of concurrency, making it ideal for use on current peta-scale and future exa-scale platforms. Additional functionality should be able to be built on top of this approach, including ab initio molecular dynamics, strong correlations via dynamical mean-field theory, core-hole spectroscopy, etc.

Given these advances in real space methods it seems sensible to pursue the development of $O(N)$ electronic structure approaches targeted towards large scale materials systems [321]. Additional functionality should be able to be built on top of these approaches, which include ab initio molecular dynamics, strong correlations via dynamical mean-field theory, core-hole spectroscopy, etc. We anticipate that these approaches will be based on sparse linear representations of the electronic structure problem, which will allow for large-scale simulations. For metallic systems, the $O(1)$ metallic states near the Fermi level could be separated out using a Wannier orbital transform. With these approaches large numbers of 
atoms can be treated on a single core processor and equally important, they can be combined with preconditioned sparse iterative methods, which contain a high degree of concurrency making the ideal for parallel applications on current peta-scale and future exa-scale platforms. The approach will be based on the density matrix formulism, which eliminates the need for costly $O\left(N^{2}-N^{3}\right)$ orthogonalizations.

A drawback of these types of methods is that the crossover from traditional $O\left(N^{3}\right)-O\left(N^{2}\right)$ to $O(N)$ methods is so large that they will not become cheaper until very large systems that require very large computational resources. An alternative $O(N)$ approach is to use Orbital Free Density Functional Theory (OF-DFT) [322-325]. This method has been shown to be able to calculate with modest computational resources $10^{6}$ atoms. Unfortunately, despite over a decade of work, this method is still not accurate enough to calculate semiconducting systems reliably. It is uncertain whether this type of method will every become accurate enough to study CdTe and CZT materials, however, recent work on using nonlocal potentials with OF-DFT may be worth exploring [326].

\subsubsection{Accurate energies of charged defects}

There is considerable ambiguity regarding the energetics of native defects and their clusters in CdTe and CZT. It is generally thought that Cd vacancies and Te antisites (and a clustering of them) are the most important in CdTe and CZT. Moreover these defects are often charged. A crucial step in the understanding of these systems is to determine accurate structures and thermodynamics for these intrinsic defects.

There have been many studies of defect and absorption energetics of CdTe and CZT using DFT [230, 327-344]. Even though DFT has been very successful in predicting structures, properties and reactivities for a wide variety of molecular and solid-state systems, the accuracy of the defect energies from DFT has not been rigorously validated from experiment or higher levels of ab initio theory. This is due in part to the fact that only the most efficient DFT methods can be used because very large supercells (>64 atoms) are needed to achieve full three-dimensional relaxation. A cause for concern is the fact that a number of breakdowns are known to exist in lower levels of DFT, such as small band gaps, inability to localize spin or charge densities, positive energies of the highest occupied molecular orbital in anions, and low reaction barriers.

The current consensus is that a number of problems associated with most approximate DFT exchange-correlation potentials can be attributed to their failure to completely cancel out the selfinteraction energy. This means that these approximations result in electrons partially seeing themselves. In mathematical terms, this means that approximate exchange-correlation functionals, $E_{x c}$, do not satisfy the equation

$$
\frac{1}{2} \iint \rho^{1}(\mathbf{r}) \rho^{1}\left(\mathbf{r}^{\prime}\right) d \mathbf{r} d \mathbf{r}^{\prime}+E_{x c}\left[\rho^{1}, 0\right]=0
$$

for any one-electron density, $\rho^{1}$ [345]. It is not known how much importance to place on these extra interactions. Experience has shown that many of the failures associated with the erroneous self-interaction term can be corrected by approaches in which the DFT exchange-correlation functionals are augmented with some fraction of exact exchange (hybrid DFT, e.g. B3LYP and PBE0) [313]. These methods have been fairly successful in many problems in molecular chemistry and solid-state physics. For example, they are able to yield localized charged states, which have posed problems for conventional DFT. They are also known to restore size consistency for systems where conventional DFT functionals are incorrect, 
and they significantly improve the band gaps and spin structure of solids, reaction barriers and NMR shielding constants.

Surprisingly there have been very few hybrid DFT calculations performed for native defects. This is likely the result of that hybrid DFT is significantly more expensive than conventional DFT. With the advent of machines that are 1 million times larger than current desktop machines along with recent algorithmic developments these costs should be able to be overcome quite readily.

Besides hybrid DFT, even higher levels of electronic structure are becoming possible for the calculation of native defects [346]. Many methods of improving the level of approximation of the many electron Schrödinger equation have been proposed (e.g., MP2, or CCSD(T) or Quantum Monte Carlo methods) and been shown to provide sufficient accuracy. These approaches are extremely computationally intensive (e.g., the method considered to be most accurate for ground state calculations, MP2, scales as $\mathrm{N}^{5}$, where $\mathrm{N}$ is the number of electrons). Today QMC calculations are likely feasible for a subset of native defects, and in the near future MP2 level calculations should be come feasible for up to 100 atoms. The major hindrance to doing these calculations today is that the software that implements these theories is primarily for molecules and clusters. With this type of software these higher-level calculations are viable up to 100 atoms. The software which implements these higher level theories is still in their infancy for the solid state, and it will likely be a couple of years before this type of software is reliable enough to perform energetic studies for CdTe and CZT.

A possible approach to determining the need for higher levels of the electronic structure theory is to use the molecular codes coupled with embedding approaches. A drawback of embedding approaches that an error is introduced, however, if used well these errors should be on the $0.1 \mathrm{eV}$ scale. Experience with the large-gap semiconductor, $\mathrm{SiC}$, is that embedding approaches with high-level theory can produce corrections as large as $1 \mathrm{eV}$. Examples of embedding approaches can be found in the following references [311, 346-350].

Another practical issue one has to deal with in calculating accurate thermodynamics for point defects is that the calculations are usually performed using periodic boundary conditions. Periodic and aperiodic systems are very different within a periodic boundary condition $(\mathrm{PBC})$ framework and this is compounded further if the system is charged (for example, charged defects, charged ions, etc.) [351]. The electrostatic energy in these systems is, in principle, divergent. A standard approach to deal with this issue is to impose a charge-neutrality condition via a uniform charge background. This implicitly introduces a jellium background. Makov and Payne have shown that this procedure results in errors that go as $L^{-1}$ for charged systems and $L^{-3}$ for isolated neutral systems in three dimensions where $L$ is the cubed root of the unit cell volume. One approach to minimize these errors is to use the scheme developed by Leslie and Gillan [352] and further improved by Makov and Payne [353]. They derived an analytic expression for the electrostatic correction between charged unit cells as follows:

$$
E_{\text {Makov-Payne }}=E_{\text {Total }}-\frac{q^{2} \alpha}{2 \varepsilon L}-\frac{2 \pi q Q}{3 \varepsilon L^{3}}+O\left(\frac{1}{L^{5}}\right)
$$

where $E_{\text {Total }}$ is the calculated energy of the charged cell, $\alpha$ is the Madelung constant for the lattice, $q$ is the total charge of the cell, and Q is the quadrupole moment of the cell given by

$$
Q=\int_{\Omega} r^{2} \rho(\mathbf{r}) d \mathbf{r}
$$


This approach has been used by Aberg et al. in their recent DFT study of defects in CZT alloys [334]. A drawback of this method is that a dielectric constant has to be estimated [353], although for many materials this constant is known. Another approach is to use an approach suggested by P. Schultz [354]. In this approach a model density that contains the defect charge is introduced. Using this density the total density of the system is then written as

$$
\rho(\mathbf{r})=\rho_{M}(\mathbf{r})+\rho_{\Delta}(\mathbf{r})
$$

The coulomb energy of the system is than determined as follows

$$
\begin{aligned}
\frac{1}{2} \iint \rho(\mathbf{r}) \rho\left(\mathbf{r}^{\prime}\right) d \mathbf{r} d \mathbf{r}^{\prime} & =\frac{1}{2} \iint \frac{\rho_{M}(\mathbf{r}) \rho_{M}\left(\mathbf{r}^{\prime}\right)}{\left|\mathbf{r}-\mathbf{r}^{\prime}\right|} d \mathbf{r} d \mathbf{r}^{\prime} \\
& +\frac{1}{2} \iint \frac{\rho_{\Delta}(\mathbf{r}) \rho_{\Delta}\left(\mathbf{r}^{\prime}\right)}{\left|\mathbf{r}-\mathbf{r}^{\prime}\right|} d \mathbf{r} d \mathbf{r}^{\prime} \\
& +\iint \frac{\rho_{M}(\mathbf{r}) \rho_{\Delta}\left(\mathbf{r}^{\prime}\right)}{\left|\mathbf{r}-\mathbf{r}^{\prime}\right|} d \mathbf{r} d \mathbf{r}^{\prime}
\end{aligned}
$$

The first integral is determined as an isolated charge density, and the second integral is determined using periodic boundary conditions. The third integral in this scheme is finite and can be determined as follows

$$
\iint \frac{\rho_{M}(\mathbf{r}) \rho_{\Delta}\left(\mathbf{r}^{\prime}\right)}{\left|\mathbf{r}-\mathbf{r}^{\prime}\right|} d \mathbf{r} d \mathbf{r}^{\prime}=\sum_{\mathbf{G} \neq 0} \frac{4 \pi}{\mathbf{G}^{2}} \rho_{\Delta}(\mathbf{G})\left[\int \rho_{M}(\mathbf{r}) \exp (-i \mathbf{G} \cdot \mathbf{r}) d \mathbf{r}\right]
$$

Both these approaches for calculating charged defect energies, $E_{d}^{q}$, can be used input into the following formula for formation energy of a defect [351]

$$
E_{f}^{q}\left(\mu_{e}\right)=E_{d}^{q}-E_{\text {supercell }}-d n_{\text {atom }}^{d} \mu_{\text {atom }}+q\left(\mu_{e}+E_{v}\right)
$$

where the $q$ is the charge of the defect, $\mu_{e}$ is the electron chemical potential, $E_{\text {supercell }}$ is the defect free supercell energy, $\mu_{\text {atom }}=E_{\text {supercell }} / N_{\text {supercellatoms }}$ is the atomic chemical potential, $d n_{\text {atom }}^{d}$ is the change in the number of atoms to form the defect, and $E_{v}$ is the valence band maximum of the perfect crystal.

Defining these types of formation energies is obviously somewhat artificial because defects are usually as collections of defects rather than as individual defects. 


\subsubsection{Free Energy Methods}

Another approach to characterizing the thermodynamics is to directly calculate the free energy of forming a group of defects, e.g. the free energy to form a vacancy and interstitial from a perfect lattice. A number of methods have been suggested to address this problem [231]. Most of these methods attempt to sample phase space along a very efficient path between the reactant state and the product state (ideally this would be the minimum energy reaction path or MEP). In solid-state physics simulations, NEB and string methods $[355,356]$ are the most popular methods for calculating MEPs. These methods work well for simple reactions pathways. However, for complex pathways the most popular approach currently is to use metadynamics [357] which is based on a molecular dynamics or ab initio molecular dynamics simulation which biases steps to move out of local minimum and, therefore, to explore new regions. This method is quite straightforward to implement. However, for complex energy surfaces there are several barriers to its efficient implementation. The most significant challenge is the identification of the important slowly changing variables. These collective variables (CV) can be used in metadynamics searches to provide directions for the exploration of the free energy surface (FES) [357, 358]. They therefore, must lead to a reasonable projection of the motion of the system along the minimum reaction path (not necessarily known) from the reactive state to the product state. Unfortunately, identifying CVs that efficiently take the system from the reactant to the product basin is a challenge. There is another significant problem with metadynamics. As the number of CV increase (beyond two) the sampling using this algorithm slows down dramatically [357].

New methods for time integration, efficient exploration of phase space, and choosing order parameters are needed. We suggest that work in the following areas (i) Develop new parallel in time integration methods. These methods utilize information from a less precise physical model to accelerate the performance of complex models. These methods work well across low cost networks and they can be used to expand scaling of simulations with limited scaling. (ii) Develop sampling methods that support the efficient exploration of complex many-body potential landscapes. A promising class of techniques that bias the process by replicating walkers making progress and discontinuing walkers that do not have been proposed and show promise (see Figure 19). (iii) Develop dimensional reduction and uncertainty quantification methods, such as principal component analysis/principal orthogonal decomposition and machinelearning techniques to extract reliable order parameters for free energy simulations and rate determination.

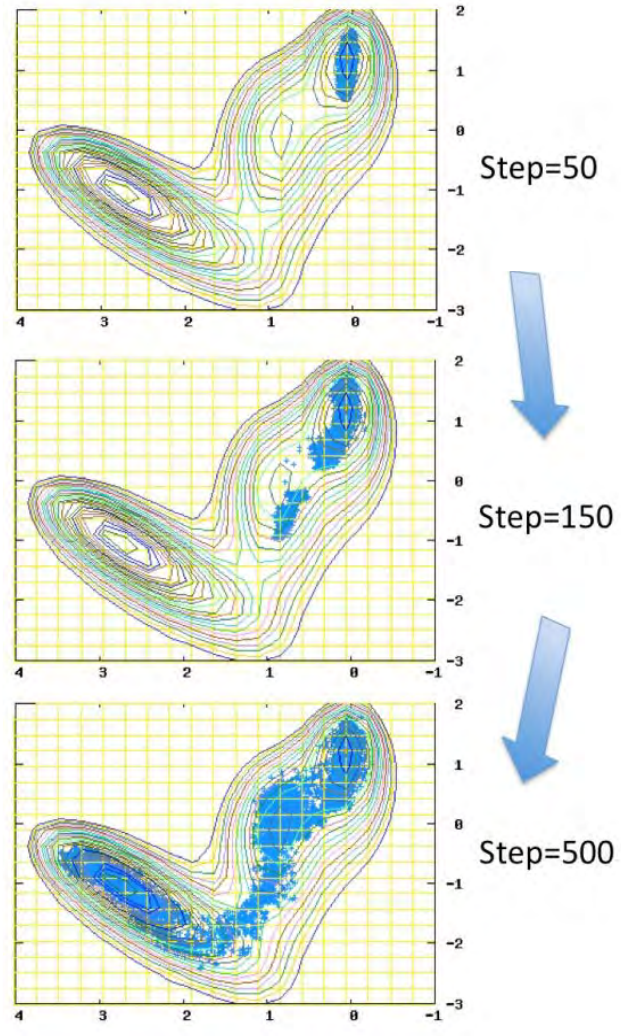

Figure 19. Sampling of the Meuller surface diffusion model. Note that the reaction paths and the three minimums have been sampled by 500 steps of the new algorithm. 


\subsection{Uncertainty Quantification and Predictive Modeling}

This section is included to consider uncertainty quantification (UQ) since UQ methods are finding general applicability in computational materials science. However, to the best of our knowledge, UQ has not yet been applied in models of crystal growth, such as for CZT modeling, where multiple time and length scales as well as inhomogeneous microstructures apply. Predictive computational materials science is broadly defined as an integrated set of mathematical modeling and experimental tools capable of computing probabilistic distributions of material properties that overlap with the corresponding measured properties even for different conditions from those used to calibrate the underlying physical models. The computed variability of properties is induced by model error, incomplete data-driven information (e.g. in constitutive equations), and loss of information during coarse-graining. The rapid developments in simulation/design methodologies including uncertainty quantification and propagation in complex systems provide us an opportunity towards predictive simulation of CZT Growth and defect modeling. However, the multiscale/multiphysics nature of the problem at hand gives rise to extremely challenging mathematical and algorithmic problems currently prohibiting predictive modeling. Several fundamental problems that need to be addressed in this context include the following as developed in the sections below.

\subsubsection{Reduced-order stochastic representations at multiple scales}

Advances in materials characterization methods [359-366] make it possible to obtain large experimental data sets that can be converted to microstructure realizations. The extraction of hierarchical structure and defect descriptors from these high-dimensional data sets is essential for predictive modeling. In addition, one is interested in probabilistic reduced-order models of microstructure. How does someone reconstruct microstructures from reduced-order representations? What microstructure (non-Euclidean) distance metrics are relevant for different properties? How do you hierarchically classify microstructures to take advantage of correlated features at different scales?

The problem of reduced representation of random microstructures can be addressed as a nonlinear dimension reduction (NLDR) problem [367-370]. What is needed are new nonlinear dimension reduction approaches, which better capture deep geometric structures, for more faithful reduced-order models. Preserving volumes $[371,372]$ rather than distances is a promising approach which has been shown that can even unfold difficult intrinsically-curved manifolds and can achieve lower dimensionalities. Preserving volumes is equivalent to preserving the probability density at each point in the microstructure space. One can also develop the desirable capability to generate microstructure samples in physical space from reduced representations, using linear-Gaussian/EM ideas similar to those of the generative topographic mapping [373, 374].

Location-dependence dramatically increases the dimensionality of the microstructure/defect input and causes the "curse of dimensionality" in a stochastic simulation. A novel data-driven bi-orthogonal Karhunen-Lo' eve (KL) decomposition strategy was recently applied to such problems [375]. The multiscale random field representing the random microstructure was decomposed into a few modes in different (macro and micro) scales simultaneously. The stochastic input complexity was remarkably simplified making stochastic multiscale modeling computationally feasible.

\subsubsection{Multiscale materials simulation}

One needs to quantify the uncertainties associated with the use of approximate electron density functionals and pseudopotentials using a Bayesian approach [376, 377]. It is essential to explore entire 
classes of functionals by considering the information loss in the coarse-graining inherent in densityfunctional theory itself. A significant challenge in coarse-graining simulations of defects is the variability of the defects themselves due to local environments. The sites around a defect have different local geometries and bonding, while each defect sees different stresses; e.g., random solutes control dislocation mobility for solid-solution strengthening [378] or cross-slip [379]. We need a stochastic multiscale mathematical approach to quantify the variability of defects in a material that control variability of mechanical properties: dislocations, dislocation subgrains, second-phase particles, crack tips, interfaces, and boundaries. In addition, non-intrusive uncertainty quantification techniques are needed to produce probability distributions of defects and properties using the deterministic multiscale simulators. These methods seek to integrate the development on physical models of defects with adaptive sparse grid collocation methods [380], HDMR approaches [381] as well as with Bayesian approaches [382, 383]. The last approaches appear promising since by utilizing active learning they require a minimal number of direct simulations for different realizations of the "local defect environment". Attention needs to be given to modeling defects in an interface [384-386], introducing flexible boundary conditions for relaxation of defects and using local electronic information of defects.

\subsubsection{Means to systematically coarse grain while preserving essential statistics}

Coarse graining of the underlying multiscale problem needs to be seen as a probabilistic inference problem in essence quantifying the information loss during coarse graining at the various scales. In addition, one needs to explore the correlated nature of the microstructures and propagate this information to predict properties. These issues arise at all scales including electronic structure or atomistic simulations. Indeed, density-functional theories and interatomic potentials can be viewed as coarsegraining the many-body electronic problem. Can we use entropy-maximizing inference models to quantify the uncertainties in extracting materials properties therefrom?

Coarse-grained models of complex, many-body systems are useful in at least two ways. On one hand, they can enhance our understanding of physical procedures. On the other hand, their evaluation requires much less computational resources than the full model allowing us to investigate problems that would be otherwise untouchable. They are a necessary component for the solution of realistic multi-scale/multiphysics CZT growth simulations. The construction of coarse-grained models based on mathematically sound grounds has been the goal of many recent efforts. The most popular techniques are Boltzmann inversion [387, 388], inverse Monte Carlo [389, 390] and force-matching methods [391, 392]. One of the most recent developments is the relative entropy framework [393]. In this approach, one defines a pool of candidate coarse-grained potentials and selects the one that minimizes the relative entropy between induced Boltzmann distribution of the coarse-grained system and the one that is based on the potential of mean force. The intuitive appeal of the relative entropy approach rests on the fact that it is trying find the coarse-grained potential that best replicates the true global statistics of the coarse-grained system as opposed to optimizing for particular statistics or matching the mean forces. Furthermore, its generality makes it applicable to problems that can potentially go far beyond the realm of traditional approaches [394-397]. It is a method that certainly allows integration of probabilistic approaches to coarse graining.

The greatest obstacle in applying this technique to realistic problems is that it requires the solution of a very difficult optimization problem. The difficulty arises from the inescapable presence of noise in the gradients of the optimized quantity (i.e. the relative entropy). These gradients include expectations with respect to the probability density of the coarse-grained system and can only be evaluated using a Monte Carlo procedure. When utilizing deterministic optimization schemes, one has to decrease the gradient 
noise to acceptable levels in order to ensure numerical stability. One can restate the optimization problem of the relative entropy method as a stochastic optimization problem. Thus stochastic optimization schemes can be considered that can deal with noisy gradients, eliminating the need for long Monte Carlo simulations and making the method computationally very attractive. This approach indeed establishes a mathematical connection between the coarse-graining and stochastic optimization communities.

\subsubsection{Ability to learn complex relations between processing, properties and structures}

Since it has become practical to assemble exhaustive databases of materials properties using multiple realizations of simulation data, can we mine them to retrieve materials relations, and deduce new multivariable laws? We could hope to search high-dimensional databases to learn the relation of CZT furnace growth conditions with statistical features of defects and microstructure at the micro- and mesoscales. Can our analysis be extended to materials properties that emerge from quantum mechanics, and a complex processing history? Can the relation of the reduced microstructure representations at different scales with the material properties and processing be learned and/or explored for rapid CZT crystal growth design? What structure and defect descriptors are appropriate for different properties of the final crystal? Of interest is to explore the relationships between a material's elemental composition, its intrinsic properties, and its quantum-mechanically determined electronic structure. Can we explore the correlations between the structural data from DFT calculations and existing property databases to uncover rules that will allow the materials designer to specify an elemental composition of CZT most likely to yield a set of desired intrinsic properties?

DFT calculations generate very large databases representing GBytes of information per single atomic configuration. Though needed to compute the final physical observables, most of this information is not directly used in analyses. The information is typically boiled down into certain conceptual observables, such as the total energy, locations of the nuclei and the forces on them, the total electron density, the density of states, samples of the reduced single-particle density matrix. One can utilize charge density features in building structure databases. Using machine-learning techniques, these data sets will be crosscorrelated with information from property databases. An intriguing possibility would be to study regression maps between the bond measures [398, 399] and low-dimensional charge density descriptors [400-402] and final macroscopic observables.

Important physical properties of CZT crystals such as yield strength, elastic modulus, thermal conductivity, hole transport and lifetimes, and gamma-ray detection resolution depend on the material microstructure and defect topology. Data mining techniques are eminently suitable for crystal growth design since optimal processing paths can be selected based on available information from a large database-relating processes, properties, and microstructures. It is thus essential to advance methodologies for designing processing stages that lead to a desired microstructure or material property by mining over a database of microstructural signatures [403]. Hierarchical databases can be designed using crystallographic orientation, grain, defect and phase topologies as microstructural signatures within a database. Microstructure representation within each class can be performed using mixture of generative topographic models [404]. These reduced modes selected adaptively from the database can be used to speed up auxiliary microstructure optimization algorithms built over the database. It is the intrinsic interconnections of these problems (and the mathematical answers to them sought) that are fundamental to our approach in developing an integrated framework for predictive materials science.

A new paradigm integrating mathematical, statistical, and information science approaches is needed in addressing these key problems. Advances in integrated developments are needed that are not common 
in other areas where UQ has been applied. For example, reduced representation of polycrystalline microstructures is not a simple extension of developments in imaging since the system of interest comes with physical constraints (grain boundaries, orientations, etc.). In addition, microstructure uncertainties are coupled across the micro-, meso-, and macro-scales, where diverse physical models are employed. Even within the same physical scale (e.g. meso-scale), one needs to address issues of multi-resolution to model detailed physics of defects, grain boundaries, imperfections, etc. Simplistic integration of material point simulators with stochastic methods (Monte Carlo or collocation) is not appropriate for stochastic multiscale models and predictive science. A new paradigm needs to be developed that addresses the curse of dimensionality. Finally, as all materials scientists are aware of, not a single model is appropriate for even the same material under different processing histories. Rather than looking for a universal materials model, the Bayesian paradigm of model selection and model averaging is followed with mixture coefficients defined by posterior probabilities computed from experimental data (e.g. of defect formation).

\subsection{Time-Scale Issues for Computational Performance}

Depending on the particular model formulation (e.g., the phase field approach uses fourth-order Cahn Hilliard equations [405, 406] or system of equivalent second-order equations [407-409] to describe the evolution of the defect concentrations), and the spatial discretization and time-discretization of the equations in the formulation, different solver techniques are needed in a discrete simulation. These solvers are the computational bottlenecks in the simulation. Since both linear and non-linear systems of equations must be solved, these solvers will include linear and non-linear solution procedures, and for large-scale systems of equations. Moreover, these systems of equations must be solved at each time-step of the simulation. Efficient and scalable solvers are thus at the crux of the computer simulation capability of the crystal growth mesoscale models. To achieve this efficiency, efficient solvers for the equations at each time-step are required to reduce the total number of time-steps in the simulation. For the lower and higher-order methods, the former can be achieved using Krylov iterative methods with scalable preconditioners, such as multigrid methods. To reduce the total number of time-steps, higher-order timediscretization schemes that permit larger time-steps will be used, such as first passage time methods. Since the total discretization error is a combination of the spatial and time discretization errors, higherorder time integrators will allow a balance of this combined error using larger time-steps than if a loworder time integrator were used.

\subsubsection{Parallel Time Methods}

Another speed up can be obtained by realizing a level of parallelism by performing the time evolution itself in parallel. This may appear counter-intuitive because time evolution is causal, or a mathematical recurrence relation, i.e., $y^{j+1}=g\left(y^{j}\right)$, see Figure 20. But substantial progress in time parallelization schemes have been made during the past decade by viewing time evolution as an iterative process [410413] over time subintervals.

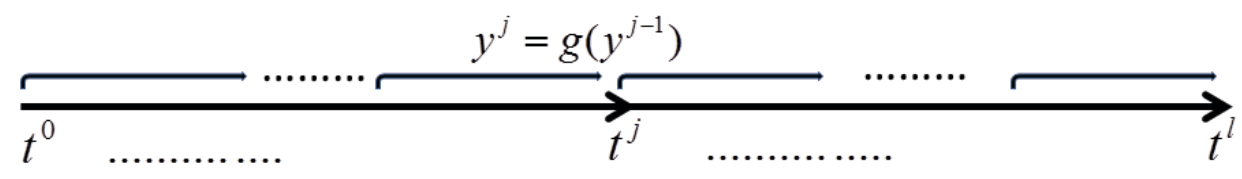

Figure 20. Time evolution viewed as a recurrence. 
To see this iterative viewpoint, in Figure 20, all that is needed for the evolution to start at $t^{j}$ is an approximation to $y^{j}$. Such an approximation is obtained by "predicting" this value in an iterative process, and such prediction can be achieved through a multi-level predictor-corrector time integration that uses coarse and fine time-step sizes, with the fine time-step being the target discretization. To illustrate this, consider Figure 21. In this diagram, a coarse time-step predictor method is propagated. Once this propagation has reached a coarse subinterval boundary, an approximation is available to start the fine time-step corrector integrator. Once this two-level predictor-corrector has completed over the full time interval, since the fine time-step is the target time discretization, this process is repeated but on an adjusted equation that incorporates the fine corrector approximations $[411,413]$. The fine corrector propagations can be done on parallel. This algorithm is called the parareal method [411] and its parallel efficiency is examined in [413].

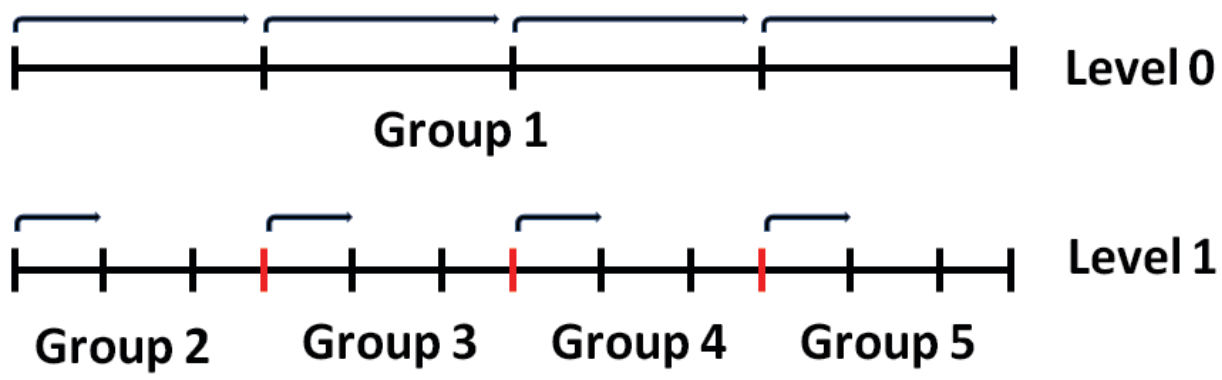

Figure 21. Parareal- two-level predictor-corrector time integration.

\subsubsection{First Passage Time}

Recently, a new algorithm for implementing a first-passage time solution for defect migration to absorbing sites, or sinks, in materials has been developed and implemented in a novel kMC scheme [414]. Tracking the most mobile defect, typically an interstitial-type defect, requires a small time step to resolve the migration. First passage approaches take advantage of the fact that mobile defect diffusion that can be treated using first-passage methods using probability density functions operating on defect concentration gradients can be computed using larger time steps, the actual first passage time. The first-passage probability is used to update mobile defect migration $[414,415]$ based on the spatial distribution of sinks. This allows defect-tracking models to efficiently treat the transport of mobile defects to defect sinks. A large speed-up can be obtained by replacing the diffusion equation with a first-passage time solution.

\subsection{Semiconductor Response Function for Microstructural Effects on Gamma-Ray Absorption and Electron-Hole Transport}

A suitable long-term goal for CZT crystal growth modeling is to develop defect structural models for evaluating the response of defective CZT crystals, focusing on the effects of dislocations, point defect clusters, and Te-particles. This type of response model is required in order to understand the role of asgrown defects in determining the energy resolution of the CZT crystals. Without this information, it will be very difficult or impossible to optimize growth conditions to achieve a desired microstructure if the goal is not known or understood. It is anticipated that a tradeoff between low Te-particle densities and low dislocation densities will be necessary to achieve optimal energy resolution for CZT radiation detectors. 
A general approach for generating a response model has been developed at PNNL and SNL that consists of using developed interatomic potentials together with ab initio methods to develop accurate structural models of extended defects in CZT and to determine their effects on charge generation and transport [4-6]. The approach employs a Monte Carlo method for tracking the generation and transport of individual electrons and holes The Monte Carlo method uses several input parameters, which can be obtained from ab initio calculations. Using these parameters, the Monte Carlo machinery allows the simulation to probe the effects of the heterogeneous structure of the defective crystals on detector properties, such as the lifetime-mobility product and energy resolution. Specifically, this response modeling makes use of an advanced Monte Carlo code Northwest Electron and Gamma Ray Interactions in Matter (NWEGRIM), which has been developed at PNNL [416-419]. NWEGRIM simulates the generation and spatial distribution of information carriers created by gamma rays and fast electrons in detector materials and has been thoroughly tested for binary and ternary semiconductors and scintillators. For example, NWEGRIM has been used to determine the intrinsic properties of pure semiconductor materials such as $\mathrm{Si}, \mathrm{Ge}, \mathrm{SiGe}$ and CZT [417-419]. The calculated mean energy per electron-hole pair, $W$, and Fano factors at $662 \mathrm{keV}$ for these materials are in excellent agreement with experimental data, as shown in Figure 22(a). These results strongly suggest that the current capabilities developed at PNNL can be applied to study more complicated materials such as defected CZT.

In addition, an important feature of NWEGRIM is its ability to calculate the spatial distribution of electron-hole pairs generated during the energy cascade. Figure 22(b) shows an example of an electronhole pair spatial distribution produced by a $2 \mathrm{keV}$ incident photon (soft X-ray) in Ge. The displayed distribution of electron-hole pairs shows some important features: the electron-hole pairs form nanoclusters along the fast electron track and the density of electron-hole pairs along the main electron track is very high. A model can be developed to expand these capabilities to include the effects of the presence of structural defects on the electron-hole pair generation, their transport under electric fields using a kinetic Monte Carlo transport code of electrons and holes, and the response signal in CZT, which would constitute a first-ever model of the effects of microstructures on the CZT response to gamma rays. This would provide the needed target microstructure to enable the crystal growth modeling to be ultimately successful in helping to select optimal growth conditions to achieve a target microstructure.

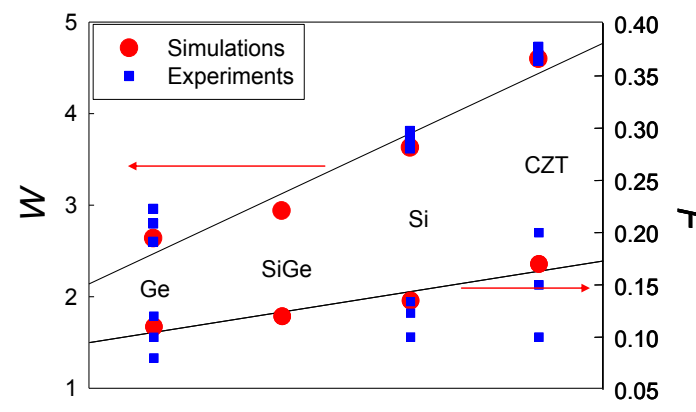

(a)

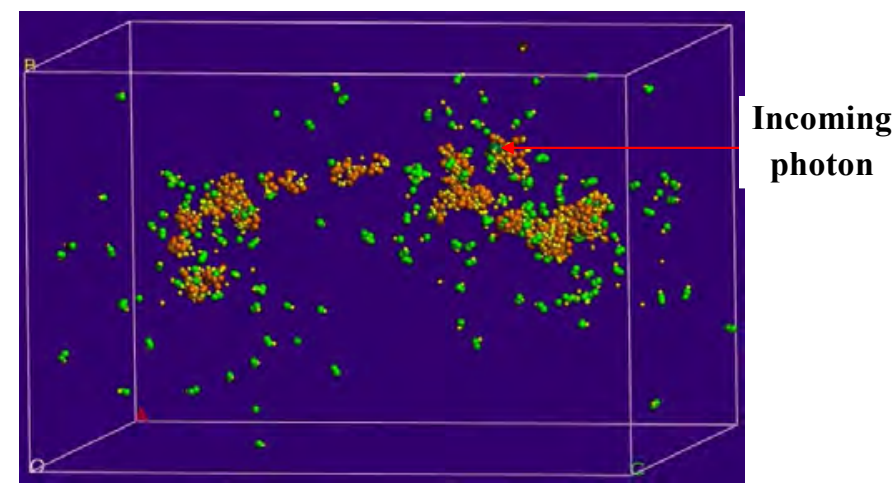

(b)

Figure 22. (a) W values (eV) and Fano factors, F, as calculated in Ge, Si, SiGe and CZT, and (b) example of a simulated spatial distribution of electron-hole pairs in Ge, where large spheres represent electrons and small yellow spheres indicate holes. 


\subsection{Revisit the Problem Statement}

\subsection{Discussion of Suggested Approach}

The suggested approach as outlined using the 6 levels in Section 3 has never been accomplished before in a single multi-physics or multi-scale methodology and we do not suggest that this is possible now. What we do suggest is that if we focus on Levels 4, 5 and 6 we can develop a sophisticated SLI model of crystal growth of CZT that can relate the growth process, including defect production, to furnace growth parameters in such a way that growth could begin to be optimized. However, simply because we can envision such a process does not mean that we know how to construct such a model today. There are significant gaps in our knowledge that will not allow this to be done without some breakthroughs. As we step through this discussion we will try to mention the gaps but they will be collected in more detail in Section 5.2.

The desired model should be able to inform as to the effects of changing furnace growth conditions or initial conditions on changes in crystal quality, which is the desired goal. However, any such problem solution is hampered by a lack of a target crystal state. Without a target state then it cannot be determined, without actually growing and testing many crystals, what the effects of optimized furnace or initial growth conditions are. This lack of knowledge hinges on the current inability to know the cause(s) of loss of detector accuracy due to crystal defects. We don't know precisely what is causing the lack of reproducible energy accuracy in as-grown CZT so that prescribing more optimal growth conditions seems to be far-fetched. This is perhaps one of the most immediate gaps that we need to address.

For convenience we reproduce Figure 1 here as Figure 23. This is the model that we desire to be able to compute SLI growth over several hundred microns, at least, for a total real-time computation over several hours in order to capture the essential SLI movement and concurrent defect formation in the wake of the moving SLI. This model can, in principle, be coupled to furnace growth parameters through boundary and initial conditions. In order to accomplish this, we envision the need to capture the correct dependencies of SLI motion as a function of $X_{i}(r, t)$, where $X_{i}$ is each of the following: 1) Temperature field, 2) Concentration field, 3) Liquid velocity field, 4) Liquid structure field, 5) Stress field, and 6) SLI configuration.

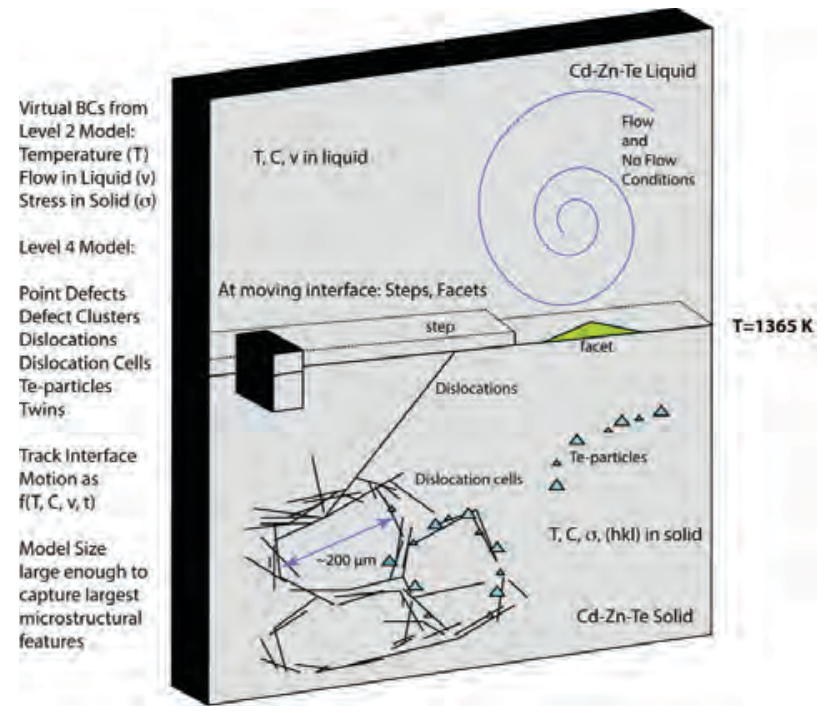

Figure 23. Schematic drawing of fully 3D, fine-scale model of CZT solid-liquid interface (SLI). 
The essence of the approach using Levels 4 to 6 are that we can work at Level 4 using data obtained at Levels 5 and 6 in terms of key thermodynamic data, such as configurational energies, mobilities of critical objects, nucleation rates of critical objects, and accurate knowledge of liquid concentrations and structures at the SLI. Hereafter, for the sake of simplicity, we constrain our discussions to a hypothetical (111)-oriented CZT interface with steps or terraces. The ability to solve this problem contains the necessary complexity to address the critical SLI simulation issues inherent in CZT modeling and is entirely relevant to seeded CZT growth. Not only that, but this problem also contains the essence of SLI growth instabilities, which is a necessary component of any SLI model of CZT growth, so that via step bunching on a moving (111) SLI we can treat breakdown of this growth direction into a unstable polycrystalline growth. Figure 24 illustrates this problem as a prototypical growth instability problem in VGF CZT growth systems. Not only is such seeded growth prone to these instabilities but similar defect densities are present even in the (111)-oriented single crystals prepared from such growths. This growth instability was linked to furnace power fluctuations but remains a typical simulation issue.

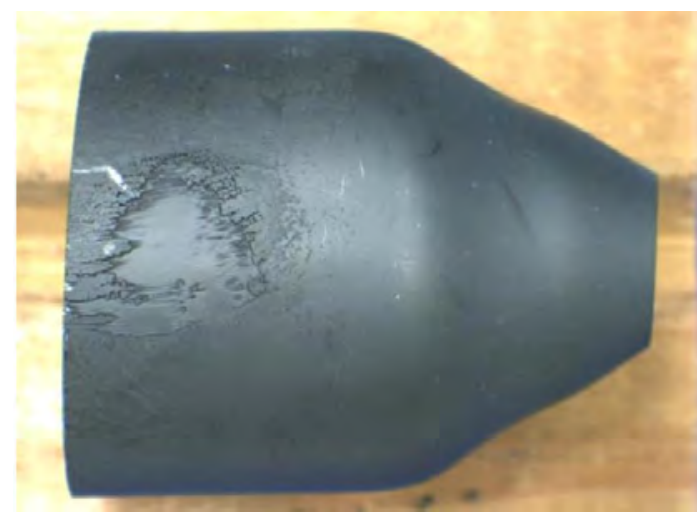

(a)

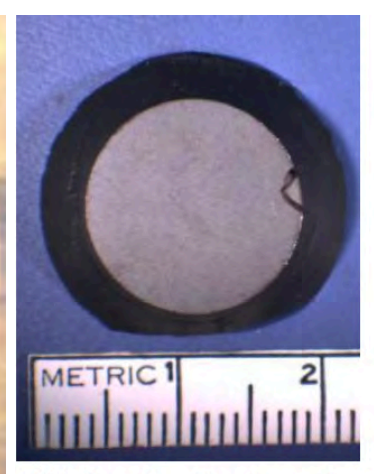

Wafer -3-Bottom view

(b)

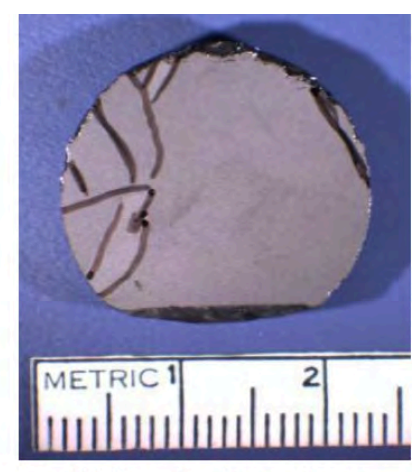

Wafer -3-Top view

(c)

Figure 24. As-grown (111)-seeded growth CZT boule and wafer slices. Shown in (a) is an as-grown (111)-seeded growth CZT boule, in (b) is a view of a wafer sliced near the tapered growth tip showing almost complete retention of (111) orientation (implied by lack of drawn-in boundaries) and (c) the same slice but the opposite side of the slice located further up the growth direction showing gradual development of polycrystalline growth (showing drawn-in boundaries). The (111)-seeded orientation was initially stable in (b) but gradually becomes unstable relative to polycrystalline growth in (c). ${ }^{4}$

Thus, the simulation reduces to an initially structured (111)-oriented interface of CZT in contact with a specified CZT liquid at $1365 \mathrm{~K}$ under an imposed thermal gradient from the VGF furnace system. The simulation cell is as shown in Figure 23 with boundary and initial conditions obtained from some unspecified furnace thermal model. The (111) solid surface contains some initial configuration of steps, ledges, and terraces consistent with step nucleation and growth kinetics and with (111) interface thermodynamics. The CZT liquid contains a velocity field, a concentration field, and a liquid structure field consistent with the initial conditions and imposed boundary conditions. The model allows continued (111) growth via steps, ledges, and terraces, or step bunching and unstable (hkl) growth. The SLI moves at approximately $0.5 \mathrm{~mm}$ /hour in real time to match typical VGF growth rates. Repeated simulations are

${ }^{4}$ All images and drawn-in boundaries are courtesy of Dr. Ralph James of Brookhaven National Laboratory in a presentation "High-Quality CZT Crystals", RadSensing2012 held at Sandia National Laboratory, Albuquerque, NM, June 5, 2012. 
run with 1) varying initial conditions and with varying boundary conditions or 2) with some randomness associated with uncertainties in material parameters in order to explore statistical growth events and to determine the overall sensitivity of the simulation to various growth variables.

The relevant assumptions of such a model are 1) a statistical representation of a (111)-oriented CZT interface in equilibrium with a corresponding CZT liquid during solidification at $1365 \mathrm{~K}$ can be computed, 2) energies and mobilities of objects and structures on the (111) SLI are known or can be computed, 3) nucleation rates of objects and structures on (111) SLI are known during solidification, 4) liquid concentrations and liquid structure models can be computed with enough accuracy so that SLI motion can be tracked as functions of these variables, 5) mechanisms of Te-ingress are known and computable, and 6) the relevant point defect and extended defect physics is known and can be used with knowledge of the solidification stress field to compute the necessary rearrangements during growth.

The methodology suggested by this approach is not really new or novel except that the application of UQ to solidification modeling in this context has not been realized. The methodology suggested here relies on information passing from one level to another level with corresponding calculations regarding structures and mobilities appropriately coarse-grained as we proceed from Level 6 to Level 4. However, significant development is required for a Level 5 model to reflect accurately the Level $\mathbf{6}$ atomistic details that are necessary. This is a typical coarse-graining problem that appears in many simulations.

A number of research and knowledge gaps naturally appear in this discussion but some may require additional explanation as provided below. In this discussion of research and knowledge gaps the focus is on Levels 6 to 4, with Level 3 requiring additional research that is also addressed but not as fully as that for the levels up to Level 4. Mention is made of some research gaps for Levels 1 and 2 as necessary but these are fine details at these levels that may not have impacts at Level 4.

\subsection{Identification of Knowledge or Method Gaps in Suggested Approach}

The gaps are arranged according to time and length scale and are denoted as either general (G) or specific (S) according to whether or not the gap in question is a general modeling and simulation gap or is a CZT-system specific gap.

\subsubsection{Atomistic/Electronic Structure - Level 6}

Gap 1. There is considerable ambiguity regarding the energetics of native defects and their clusters in CdTe and CZT. It is generally thought that $\mathrm{Cd}$ vacancies and Te antisites (and a clustering of them) are the most important in CdTe and CZT. Moreover these defects are often charged. A crucial step in the understanding of these systems is to determine accurate structures and thermodynamics for these intrinsic defects, defect clusters, and more extended defects. (S)

a. New and more accurate methods of treating charge transfer and charge localization are required for $\mathrm{CZT}$ defects.

Gap 2. Large $O(N)$ DFT methods will need to be developed to treat larger atomic systems for defects, defect clusters, extended defects, and SLI morphologies since semi-empirical methods lack the ability to treat charged defects. (G)

Gap 3. Develop sampling methods that support the efficient exploration of complex many-body potential landscapes. A promising class of techniques that bias the process by replicating walkers making progress and discontinuing walkers that do not have been proposed and show promise. This would improve the accuracy with which complex defect energies are known. (G) 
a. This becomes a significant issue for compounds and alloys. There is a reason that the prior significant multi-physics models have been done for Si and not for CdTe or CZT.

Gap 4. Develop dimensional reduction and UQ methods, such as principal component

analysis/principal orthogonal decomposition and machine-learning techniques to extract reliable order parameters for free energy simulations and rate determination. (G)

a. This would allow the correct coarse-graining to occur with reliable energies and rates for

\section{Levels 5 and 4.}

Gap 5. There is currently no reasonable IAP for CdTe or CZT that can be used to develop precise mechanistic concepts, to calculate required energetics, and to capture long-range liquid structures. This gap anticipates that there are problems that cannot be handled using current DFT methods. (S)

a. As one moves from DFT to a semi-empirical IAP the ability to treat larger models and more complex defects is enhanced but the ability to handle charge transfer is greatly reduced.

b. Can we create DFT surrogates? Can we develop other parameterizations, such as nonparametric forms or use cross entropy minimization?

\subsubsection{Near Atomic, High-Resolution SLI Models - Level 5}

In moving from Level 6 to Level 5 we face the first of a set of gaps that are similar as we move from level to level and that deal with coarse-graining. The gap is general in the sense that there is no set methodology to do this and is also specific to CZT since any coarse-graining must account for problem specificity. This gap revolves around the need to create surrogate representations at each level of what is essentially an atomistic process, namely crystal growth from the liquid. Since it is not yet possible to treat this problem at large enough time and length scales we must create accurate surrogate representations of these processes. How are these accomplished? There are specific methods to consider, such as phase field, $\mathrm{kMC}$, level set, etc., and there are mechanistic details to consider, including how and whether to coarsegrain certain objects and events.

Gap 6. How do we coarse-grain or homogenize fine-scale events into a surrogate representation at a coarser scale so that enough accuracy is retained to achieve longer time simulations and physically larger models? This is the crux of one of the real difficult problems we face in describing crystal growth. (G)

a. Given the ability to calculate free energies of surface steps, ledges, and terraces on a hypothetical (111) SLI can we construct a representation of such a surface at equilibrium with a melt at $1365 \mathrm{~K}$ ? How does it grow? How does solidification actually occur at this surface given the details of the SLI? This is a critical gap for any suggested model of SLI movement and solidification. This is also the first of the coarse-graining problems that we face in constructing a model. Here we envision constructing a discrete structural model that relies on continuum fields or order parameters. The method might be $\mathrm{kMC}$ or level set or phase field. Energies and rates of defects are required. (G)

b. Can we construct a representative (111) SLI based on Level 6 computations of free energies and nucleation rates? (S)

i. What objects need to be included in this (111) SLI representation?

ii. How accurately do we need to know the rates and energies? This is a UQ question.

c. We need to understand the effect of conditions at the moving SLI, such as local chemical concentration, temperature gradients and fluctuations, melt convection and liquid flow, 
liquid-phase structure, and surface stresses on the nucleation rate and mobilities of steps and on the impinging flux available for growth. (S)

i. How do we compute nucleation rates? Can we use classical nucleation theories?

ii. Can we compute the necessary mobilities of the growth steps and ledges?

iii. How do the velocity, concentration, and structure fields of the liquid at the moving SLI affect the SLI mobility?

d. Modeling SLI morphological instability during solidification is a gap. (G)

i. How do we integrate the thermodynamic and kinetic properties of interfacial defects, local growth conditions, and rate processes of defect nucleation into a model to simulate the SLI morphological evolution?

ii. Higher level models need the database on the dependence of SLI morphologies, SLI morphological instability, and SLI mobility on growth local growth conditions. Can we build the database?

iii. How do we do phase field or level set models with uncertainties in growth conditions, flow, parameters, etc.? Should we predict statistics of growth rather than a unique morphology and or unique state of stability conditions?

\subsubsection{The SLI Growth Model with Concurrent Defect Generation - Level 4}

Gap 7. The Level 4 model operates at the micron-scale and covers millions of cubic microns so that the defects of interest to CZT growth are treated, in principle, namely 1) Te-inclusion and precipitate formation, 2) dislocation and twin generation, 3) Te-dislocation interactions, 4) dislocation cell formation, and 5) any and all growth instabilities and morphologies at the moving SLI interface. The formation of defects is directly related to the SLI morphology and to the interactions between CZT species and the defects, including Te-dislocation interactions and all of the stress-induced annealing that occurs in the solid behind the moving SLI. This requires the fine details of Level 5 be coarse-grained to Level 4. Can we extract the statistics of defects rather than morphology - which statistics are needed? Can we do this? (G)

a. This level requires knowledge of stresses that can cause dislocation and twin generation, and concurrent Te-ingress along extended defects. This appears to require that we develop a physics-based model integrating the generation of different defects and concurrent microstructure evolution. Can we integrate the microstructure evolution model into existing models, such as the Alexander-Haasen model [420, 421], crystal plasticity models [214, 215, 218], and Sinno [43]. (S)

b. A physics-based model typically needs the thermodynamic and kinetic properties of bulk materials and defects as a function of temperature and concentration, and the question is do we have all the data that is needed? This requires a sensitivity analysis to determine what is important or what needs to be computed accurately. (G)

i. For bulk materials: Elastic constants, thermal expansion coefficients, thermal conductivity, solid-state diffusivities, liquid diffusivities, and chemical free energies of different phases.

(1) For defects: Energy and mobility of the SLI, configurations and energies of crystal lattice defects, defect migration energies, lattice, dislocation, twin, and GB diffusivities, defect interaction energies (trapping, binding, etc.), structure of extended defects such as dislocation networks, mechanisms of defect formation 
such as Te ingress, solute diffusion along dislocation cores, direct trapping of Te inclusion at SLI, and homogeneous and heterogeneous Te precipitation.

Gap 8. A UQ treatment of a large set of variables, such as the list above, is extremely daunting. How do we prioritize these variables and how do we represent them in coarse-grain models? Some of these require treatment as essentially random variables, but which ones? (G)

Gap 9. Lack of a growth target for CZT crystals based on a gamma-ray absorption response function model (Section 4.8). This is a gap since without a response function model one cannot know when a suitable target microstructure is reached without growing and testing many crystals. It would be extremely cost effective to have a target for the SLI growth model to aim at. (S)

\subsubsection{Grain Competition and Nucleation - Level 3}

Gap 10. For seeded growth into a melt we would need to know the growth kinetics of the various orientations. This is treated in Level 4. (S)

Gap 11. For unseeded growth we need to develop models of nucleation or at least arrive at a defensible starting point for the simulation. (S)
a. Can we develop algorithms for stochastic microstructures as starting points?
b. We would need growth kinetics for all orientations.
c. We would need surface energies and GB energies to allow for GB competition.
d. We would need mechanisms for attachment and orientation selection so that grains either grow or are nucleated to change orientations.
e. We would need good models of twin formation during growth.
i. There are existing models but unsure how robust they are.

\subsubsection{Global and Local Furnace Models - Levels 1 and 2}

Levels 1 and 2 furnace models have not yet been treated using UQ methods. If certain variables have been studied with enough trials, such as crucible rotation or applied magnetic fields, then UQ can be used to solve the inverse problem of furnace design to achieve some critical growth conditions. Alternatively, can we consider a furnace growth condition that essentially removes liquid convection, turbulence, and container constraints from the model so that we can more easily study SLI growth at finer scales?

Gap 12. Apply UQ methods to CZT furnace growth data. (S)

Gap 13. There is a lack of systematic study of turbulent flow in complex melts, and in particular, in CdTe or CZT melts. This could have consequences on liquid phase transport and gradients in the liquid. (S)

\subsubsection{UQ Physics and Statistical Representations}

Gap 14. There is a need to learn about quantifying microstructure uncertainty using limited experimental or simulation data - producing probability models for the underlying microstructures at different scales. These methods also include directly linking microstructures to crystal properties. (G)

a. This is significant for crystal growth problems where large growth data is limited.

b. UQ using limited data (epistemic uncertainty induced by the limited data).

Gap 15. Quantifying uncertainties in atomistic potentials and their relevance to macro- or meso-scale properties is needed to understand both their limitations and their overall accuracy. (G) 
a. For example, we can use DFT models to generate structures and ask "How close to those structures and energies does a good IAP need to be?" (G)

b. For CZT this is likely to focus on charge transfer. (S)

Gap 16. Stochastic coarse graining - UQ in multiscale simulations. In particular the inverse problem:

Can we identify what multiscale features contribute to variability of observables so we can focus our attention on more accurate simulations at those scales, or better experiments at those scales? We seek to create surrogate predictive models at each scale that allow us to say something about our confidence in our predictions from limited simulations. (G)

a. This goes to the heart of one of the problems with modeling CZT SLIs and knowing what is important to include or what can be excluded from the models. (S)

b. Can we hierarchically write down the sources of uncertainty that contribute in the performance of CZT? Is it control of macro-thermal-flow conditions or inherent randomness on the micro- and meso-scales? If we can predict the contribution of each of these sources to the final desired CZT crystal properties then one can focus resources in minimizing relevant uncertainties. (S)

\subsubsection{General Computability Issues}

Gap 17. See Gap 2. This is a common problem but for CZT it is a critical gap.

Gap 18. Develop improved time integration methods for multiscale simulations such as the one for CZT. These new methods might utilize information from a less precise physical model to accelerate the performance of complex models. These methods would work well across low cost networks and could be used to expand scaling of simulations. (G)

a. These could include parallel time methods. (G)

b. These could include methods such as Equation Free methods that use coarse-graining and bursts of fine-scale simulations to learn system dynamics. (G)

c. First passage time algorithms have been developed for some reaction-diffusion problems and could be applied to cases for CZT crystal growth modeling where defect dynamics are computational bottlenecks. (S)

Gap 19. Develop improved spatial and temporal discretization methods for phase field models. This would involve use of adaptive spatial grids and adaptive time stepping for tracking order parameters having disparate time scales. (G) 


\subsection{Suggestions for Closing the Gaps (Future Research Directions)}

Constraining the focus to Levels 4, 5, and 6 still leaves us with a large number of related knowledge gaps that would have to be filled with future research in CZT SLI models to achieve the desired goal of "CZT growth optimization in melt-growth systems with improved energy resolution". The gaps range from lack of tools, such as a lack of a satisfactory DFT treatment of charge transfer or $O(N)$ DFT, to a lack of knowledge in methodology, such as how to effectively and systematically coarse-grain from finer scale data with a knowledge of error propagation. In all cases, research tasks can be identified that will address these shortcomings, but without guarantee of success in all cases. Some methods benefit from improvements in HPC resources while others may require completely new methods to be developed with uncertain success. In the following sections we suggest research directions consistent with 1) what has been done in other material systems, 2) this report, and 3) what could be done given sufficient resources based on current knowledge. It should be noted that some of the needed developments will impact many other multiscale materials systems and go beyond CZT growth models.

Any model of SLI motion during crystal growth of CZT must face the question "How does this interface grow (solidify) given the coupling between the thermal, concentration, stress, and morphological/structural fields present in the system?" At its root, any solution to this problem is envisioned to consist of an array of simple rate events that can be determined more or less precisely in energetics and kinetics so that an accurate physical model can be constructed. If the model is not detailed enough then the coupling between the furnace growth conditions and the SLI model will be too weak to be deterministic as to the SLI growth and concurrent defect generation. In other words, the model will be unable to inform as to the effects of changing furnace growth conditions or initial conditions on crystal quality, which is the desired goal. In part, as will be pointed out below, this problem solution is hampered by a lack of a target crystal state. Without a target state then it cannot be determined, without actually growing and testing many crystals, what the effects of optimized furnace or initial growth conditions are. This lack of knowledge hinges on the current inability to know the cause(s) of loss of detector accuracy due to crystal defects. We don't know precisely what is causing the lack of reproducible energy accuracy in as-grown CZT so that prescribing more optimal growth conditions seems to be far-fetched.

We set out four basic research directions or recommendations based on this report and they are numbered 1 to 4, but this is not the order of research priority that we envision. The first research direction involves a simplified SLI growth model for CZT that is consistent with closing many of the gaps outlined here but does not achieve the full goal that we have addressed in this study. The second research direction does that. However, the third research direction may be the most valuable of all and that one has to do with building a more sophisticated gamma-ray response model for CZT with an ensemble of known defects. We need an explicit link between CZT microstructure and properties to use for the CZT growth models. Fortunately, we think that this third research direction is the one most readily accomplished based on work at PNNL on energy loss models developed for scintillators, called NWEGRIM. These energy loss methods are generalizable to CZT and gamma-ray absorption leading to signal generation and energy resolution.

Finally, since this computational research appears to push the bounds of what can be currently computed there is also a need to examine algorithm improvements and this is done below within the context of the current state-of-the-art in large-scale quantum chemistry codes, and in time domain speedups. A point to consider here is the concurrent interest of ASCR in these same research topics. 


\subsubsection{Models of a solid-liquid interface (SLI) in CZT}

\subsubsection{Research Direction 1: SLI Stability - Based on (111)-seeded CZT growth}

Construct a high-resolution model of a (111) CZT surface in contact with a CZT liquid at $1365 \mathrm{~K}$ based on a detailed knowledge of the energy landscape of an equilibrated (111) surface at $1365 \mathrm{~K}$ in contact with a typical CZT liquid, which might be slightly Te-rich per usual growth chemistry. The (111) surface will consist of a statistical representation of a (111) surface containing steps, ledges, and terraces or steps based on the computed energy landscape. Each of the surface defects will have mobilities as functions of the necessary growth fields (temperature, concentration, liquid velocity, stress, and morphological) so that the (111) SLI will move in time in response to these fields. Creating and solving this model will inform on SLI stability with the assumption that maintaining this stability is tantamount to growing an improved CZT crystal. The model should operate at near-atomic resolution but with long times to allow liquid diffusion and adequate step motion. Examples of such models might be kMC, phase field, or level set. Energy landscapes require atomistic models using accurate IAPs. Uncertainty quantification should inform the new IAP and the supposed database of equilibrated (111) surfaces for this effort. A model with both phenomenological and stochastic methods is more realistic here.

1. This model will not specifically track solid-phase defects but will focus on (111) SLI stability.

a. Explore SLI instability as a function of growth rate and liquid flow rate.

i. SLI instability is envisioned to occur due to step bunching and grain nucleation.

ii. Other instabilities will have to come from physical details of the model.

2. This model addresses Gaps 1 to 5 at the atomistic scale.

a. This model requires an improved method for charge transfer for CZT to allow charged defects to be studied.

b. This model requires a robust energy landscape algorithm for any atomistic or electronic structure method. Explore relative entropy approaches?

c. This model would benefit greatly from $O(N)$ DFT development.

d. This model requires development of dimensional reduction and UQ methods to treat energy landscape coarse-graining.

e. This model requires the development of an improved IAP for CZT.

3. This model addresses Gap 6 as a type of coarse-graining problem.

\subsubsection{Research Direction 2: SLI Motion with concurrent defect generation in CZT}

Given some success in direction 1 above, a more comprehensive model can begin to be constructed. In this case, the moving SLI along with the array of fields discussed above (thermal, stress, flow, and morphological) will be tracked and incorporated into defect production rates. Nucleation of defects is treated in a self-consistent manner in this model. The most important defects are dislocations and twins, Te-particles, and point defects leading to defect clusters. This is demanding since the range of sizes are large and the time scales are substantial. A form of coarse-graining of this problem will need to be developed to go between the atomic scales and the mesoscale. It is not envisioned that a single multi-scale model can capture this range of events but that a coarse-grained approach will be developed based on phenomenological and statistical likelihoods computed from energies and rates found at more 
fundamental scales. UQ will be a necessary tool here based on correct sampling statistics and the like. The model can be constructed from Research Direction 1 above using a (111) interface as the basis.

1. This model will specifically track solid-phase defects based on (111) SLI stable growth with permitted growth instabilities.

a. Explore SLI instability as a function of growth rate and liquid flow rate.

b. Explore defect generation as a function of growth rate and continuum fields with concurrent uncertainties.

c. Nucleation of defects should be treated in self-consistent manner.

d. Defects should be self-consistently coupled as Te-particles, dislocations, and point defect clusters interact.

2. This model almost requires that charge transfer and charged defects be accurately treated.

3. This model will require an improved IAP for CZT to allow large-scale surface and bulk defects to be created and studied.

a. Both solid and liquid phases require some fine details to be studied.

b. This model will require a robust energy landscape algorithm for any atomistic or electronic structure method finding energies and transition states.

4. A robust coarse-grained method to proceed from atomistic to mesoscale needs to be developed here.

a. Can be based on UQ approach and sampling algorithms.

5. Model development likely spans from atomic to mesoscale continuum methods.

a. Largest structures necessary for defect model appears to be dislocation cells and require a large model that may be beyond current capabilities.

b. What are the statistics of the defect structures observed? Can we coarse-grain while maintaining statistical models that satisfy some statistics of the topology?

\subsubsection{Research Direction 3: Semiconductor Response Models with As-Grown Defects}

In order to make decisions regarding growth models and optimizations, it is recommended that a CZT response model be constructed based on the success of the current NWEGRIM methods employed for scintillator radiation detectors. Many of the same methods can be applied here. It is difficult to know a priori what a suitable target microstructure is without having a response model that can be used to predict the effect of as-grown crystal defects on response, especially given that this is the main goal of improved crystal growth. The task is to develop a computer model for evaluating the response of defective CZT crystals, focusing on the effects of dislocations, point defect clusters, and Te-particles. The general approach consists of employing a Monte Carlo method for tracking the generation and transport of individual electrons and holes and for determining pulse heights under applied electric fields. The Monte Carlo method uses several input parameters, which can be obtained from ab initio calculations. Using these parameters, the Monte Carlo machinery probes the effects of a hypothetical heterogeneous structure of a defective crystal on detector properties such as the lifetime-mobility product. This task can be divided into two subtasks, which respective aims are to: 1) derive the principal Monte Carlo input parameters from ab initio calculations and 2) carry out simulations of charge generation and transport in defective CZT crystals. The atomistic nature of the approach will allow the investigation of a range of densities, spatial distributions, and size distributions of Te-particles, defect clusters, and dislocation configurations. This is really a UQ problem where we have developed accurate stochastic models for the densities of defects, size distributions, etc. 
This response modeling task can make use of the advanced Monte Carlo code Northwest Electron and Gamma Ray Interactions in Matter (NWEGRIM), which simulates the generation and spatial distribution of information carriers created by gamma rays and fast electrons in detector materials and has been thoroughly tested for binary and ternary semiconductors and scintillators. For example, NWEGRIM has been used to determine the intrinsic properties of pure semiconductor materials such as $\mathrm{Si}, \mathrm{Ge}, \mathrm{SiGe}$ and CZT. The calculated mean energy per electron-hole pair, $W$, and Fano factors at $662 \mathrm{keV}$ for these materials are in excellent agreement with experimental data, as shown in Figure 22. These results strongly suggest that the current capabilities developed at PNNL can be applied to study more complicated materials such as defected CZT.

\subsubsection{Research Direction 4: HPC Algorithm Development}

This research effort can support both DFT code improvements, including higher accuracy and $O(N)$ methods, but also both spatial and temporal decompositions for current phase field methods, for example. Efficient and scalable solvers are at the crux of the computer simulation capability of the crystal growth mesoscale models since any of the proposed modeling efforts will operate right at the edge of current computability. Depending on the particular model formulation (e.g., the phase field approach uses fourthorder Cahn Hilliard equations or system of equivalent second-order equations to describe the evolution of the defect concentrations), and the spatial discretization and time-discretization of the equations in the formulation, different solver techniques are needed in a discrete simulation such as those proposed here for modeling CZT crystal growth. These solvers are the computational bottlenecks in the simulation. However, the formulation of the problem needs to be examined so that spatial adaptivity is developed, perhaps through the use of advanced multigrid or adaptive grid methods, and, perhaps, the different fields or order parameters can be decoupled. For example, thermal, stress, and concentration fields obey distinct solution time steps and could be solved using some parallel time algorithms. To reduce the total number of time-steps, higher-order time-discretization schemes that permit larger time-steps can be used, such as first passage time methods. Since the total discretization error is a combination of the spatial and time discretization errors, higher-order time integrators will allow a balance of this combined error using larger time-steps than if a low-order time integrator were used. It should be noted that some links to ASCRfunded Fast Math centers would be appropriate.

\subsection{Conclusions}

A comprehensive review of the current state-of-the-art in modeling SLI stability during crystal growth and in modeling defect evolution during crystal growth has been accomplished and used to reveal a series of gaps in our current modeling capabilities in applying such models to crystal growth of CZT. Thirteen of the gaps are in general model development issues and in crystal growth physics. Three of the gaps are in the relatively new area of uncertainty quantification, or UQ, that can be used to guide both model development and data sampling efforts for this daunting task. Two of the gaps are in computational areas having to do with increased computational efficiency for the anticipated methods.

Research Directions 1 and 2 were suggested for closing these gaps in a gradual manner for CZT, with the UQ gaps embedded within the attempts to develop crystal growth models, while the computational gaps are treated separately in Research Direction 4. A response model for gamma-ray energy resolution in defective CZT crystals was suggested as Research Direction 3.

It was recommended that a Research Direction 1 for crystal growth modeling focus on the stability during growth of a starting (111) seeded SLI for CZT. Solving this model will prove to be a substantial 
achievement and will relate, for the first time, furnace growth conditions to maintaining a stable (111) growth without competing grain nucleation. This achievement will immediately suggest improved growth conditions for CZT since (111) growth is the preferred growth state and cannot always be maintained during VGF seeded growth.

Research Direction 2 focused on the more difficult problem of simultaneously allowing SLI growth plus defect generation in the growing CZT solid. This is substantially more difficult due to uncertainties in nucleation rates of defects and unproven coarse-grain model development of highly coupled crystal growth events. For this model to be successful it must treat Te-particle genesis, dislocation generation, and point defect clustering during crystal growth. The main difficulty appears to be the long times required for the simulation and the large size of the high resolution models in order to capture dislocation cells formed during CZT cool down.

It was also noted that there is a great need for Research Direction 3 for a validated CZT radiation detector response model to be developed to allow a target CZT defected microstructure to be computed. It is difficult to proceed along a path to minimize as-grown defects if there is not an understanding of the mechanisms for degradation in energy resolution in CZT for particular types of defects. For example, it may be possible to reduce the defect levels to some threshold that will achieve near-theoretical gammaray energy resolution without fully optimizing CZT crystal growth. On the other hand, it might not be possible to achieve this resolution with current crystal growth methods. A physically based CZT response model would help answer these questions.

The computational gaps require closing in Research Direction 4 to allow larger simulations with improved accuracy, from $O(N)$ DFT with charge transfer to large-scale discrete simulations, such as phase field models. The goal of this research is to speedup computations by orders of magnitude while maintaining high spatial accuracy.

The near-term recommendations would be to fund Research Direction 1 and 3 immediately with Research Direction 2 waiting for success in RD 1. Research Direction 4 should be pursued in collaboration with ASCR, if possible.

\subsection{References}

1. Ndap, J.-O., Inclusions and Precipitates in CdZnTe Substrates, in CdTe and Related Compounds; Physics, Defects, Hetero- and Nano-structures, Crystal Growth, Surfaces and Applications, R. Triboulet and P. Siffert, Editors. 2010, Elsevier Ltd. p. 228-257.

2. Durose, K., Extended Defects in CdTe, in CdTe and Related Compounds; Physics, Defects, Hetero- and Nano-structures, Crystal Growth, Surfaces and Applications, R. Triboulet and P. Siffert, Editors. 2010, Elsevier Ltd. p. 171-227.

3. Sundaram, S.K., C.H. Henager Jr, D.J. Edwards, A.L. Schemer-Kohrn, M. Bliss, and B.R. Riley, "Electron backscatter diffraction analysis of a CZT growth tip from a vertical gradient freeze furnace," Journal of Crystal Growth, 2011, 329(1), 12-19.

4. Wang, Z., Y. Xie, L.W. Campbell, F. Gao, and S. Kerisit, "Monte Carlo simulations of electron thermalization in alkali iodide and alkaline-earth fluoride scintillators," Journal of Applied Physics, 2012, 112(1).

5. Wang, Z., Y. Xie, B.D. Cannon, L.W. Campbell, F. Gao, and S. Kerisit, "Computer simulation of electron thermalization in CsI and CsI(Tl)," Journal of Applied Physics, 2011, 110(6).

6. Kerisit, S., K.M. Rosso, B.D. Cannon, F. Gao, and Y. Xie, "Computer simulation of the light yield nonlinearity of inorganic scintillators," Journal of Applied Physics, 2009, 105(11).

7. Yeckel, A., L. Lun, and J.J. Derby, "Multi-scale crystal growth computations via an approximate block Newton method," Journal of Crystal Growth, 2010, 312(8), 1463-1467. 
8. Derby, J.J., D. Gasperino, N. Zhang, and A. Yeckel, Modeling the crystal growth of cadmium zinc telluride: Accomplishments and future challenges, in Nuclear Radiation Detection Materials, Vol. 1164: 2009 MRS Spring Meeting, April 14, 2009 - April 16, 2009, D.L. Perry, et al., Editors. 2010, Materials Research Society: San Francisco, CA, USA. p. 45-53.

9. Yeckel, A., L. Lun, and J.J. Derby, "An approximate block Newton method for coupled iterations of nonlinear solvers: theory and conjugate heat transfer applications," Journal of Computational Physics, 2009, 228(23), 8566-88.

10. Derby, J.J., L. Lun, and A. Yeckel, "Strategies for the coupling of global and local crystal growth models," Journal of Crystal Growth, 2007, 303(1 SPEC ISS), 114-123.

11. Dropka, N., C. Frank-Rotsch, W. Miller, and P. Rudolph, "Influence of travelling magnetic fields on SL interface shapes of materials with different electrical conductivities," Journal of Crystal Growth, 2012, 338(1), 208-213.

12. Yeckel, A. and J.J. Derby. Effects of a traveling magnetic field on vertical gradient freeze growth of cadmium zinc telluride. in Hard X-Ray, Gamma-Ray, and Neutron Detector Physics XIII, August 22, 2011 - August 24, 2011. 2011. San Diego, CA, United states: SPIE.

13. Rudolph, P., "Travelling magnetic fields applied to bulk crystal growth from the melt: the step from basic research to industrial scale," Journal of Crystal Growth, 2008, 310(7-9), 1298-306.

14. Kumar, V., S. Dost, and F. Durst, "Numerical modeling of crystal growth under strong magnetic fields: An application to the travelling heater method," Applied Mathematical Modelling, 2007, 31(3), 589-605.

15. Wang, Y., K. Kudo, Y. Inatomi, R. Ji, and T. Motegi, "Growth and structure of CdZnTe crystal from Te solution with THM technique under static magnetic field," Journal of Crystal Growth, 2005, 275(1-2), 1551-6.

16. Chenying, Z., M. Jiahua, W. Changjun, L. Xiaoyan, T. Jianyong, and W. Linjun, "Investigation of influence of accelerated rotating wave on solid-liquid interface in CdZnTe ACRT crystal growth," Physica Status Solidi C, 2010, 7(6), 1482-5.

17. Juncheng, L., "ACRT forced convection and its effects on solute segregation and heat and mass transfer during single crystal growth," Crystal Research and Technology, 2008, 43(4), 396-408.

18. Liu, Y.C., B. Roux, and C.W. Lan, "Effects of accelerated crucible rotation on segregation and interface morphology for vertical Bridgman crystal growth: Visualization and simulation," Journal of Crystal Growth, 2007, 304(1), 236-243.

19. Lun, L., A. Yeckel, M. Reed, C. Szeles, P. Daoutidis, and J.J. Derby, "On the effects of furnace gradients on interface shape during the growth of cadmium zinc telluride in EDG furnaces," Journal of Crystal Growth, 2006, 290(1), 35-43.

20. Lun, L., A. Yeckel, P. Daoutidis, and J.J. Derby, "Decreasing lateral segregation in cadmium zinc telluride via ampoule tilting during vertical Bridgman growth," Journal of Crystal Growth, 2006, 291(2), 348-357.

21. Sonda, P., A. Yeckel, J.J. Derby, and P. Daoutidis, "The feedback control of the vertical Bridgman crystal growth process by crucible rotation: Two case studies," Computers and Chemical Engineering, 2005, 29(4), 887-896.

22. Yeckel, A. and J.J. Derby, "Buoyancy and rotation in small-scale vertical Bridgman growth of cadmium zinc telluride using accelerated crucible rotation," Journal of Crystal Growth, 2001, 233(3), 599-608.

23. Zhang, N., A. Yeckel, and J.J. Derby, "Maintaining convex interface shapes during electrodynamic gradient freeze growth of cadmium zinc telluride using a dynamic, bell-curve furnace profile," Journal of Crystal Growth, 2012, 355(1), 113-121.

24. Marchini, L., A. Zappettini, M. Zha, N. Zambelli, A.E. Bolotnikov, G.S. Camarda, and R.B. James, "Crystal defects in CdZnTe crystals grown by the modified low-pressure Bridgman method," IEEE Transactions on Nuclear Science, 2012, 59(2), 264-267. 
25. Li, Q., M. Beilicke, K. Lee, A. Garson Iii, Q. Guo, J. Martin, Y. Yin, P. Dowkontt, G. De Geronimo, I. Jung, and H. Krawczynski, "Study of thick CZT detectors for X-ray and Gamma-ray astronomy," Astroparticle Physics, 2011, 34(10), 769-777.

26. Awadalla, S.A., J. Mackenzie, H. Chen, B. Redden, G. Bindley, M.C. Duff, A. Burger, M. Groza, V. Buliga, J.P. Bradley, Z.R. Dai, N. Teslich, and D.R. Black, "Characterization of detector-grade CdZnTe crystals grown by traveling heater method (THM)," Journal of Crystal Growth, 2010, 312(4), 507-513.

27. Roy, U.N., A. Gueorguiev, S. Weiller, and J. Stein, "Growth of spectroscopic grade Cd0.9Zn0.1Te:In by THM technique," Journal of Crystal Growth, 2009, 312(1), 33-36.

28. Chen, H., S.A. Awadalla, K. Iniewski, P.H. Lu, F. Harris, J. MacKenzie, T. Hasanen, W. Chen, R. Redden, G. Bindley, I. Kuvvetli, C. Budtz-Jorgensen, P. Luke, M. Amman, J.S. Lee, A.E. Bolotnikov, G.S. Camarda, Y. Cui, A. Hossain, and R.B. James, "Characterization of large cadmium zinc telluride crystals grown by traveling heater method," Journal of Applied Physics, 2008, 103(1), 014903.

29. Triboulet, R. and P. Siffert, eds. CdTe and Related Compounds; Physics, Defects, Hetero- and Nano-structures, Crystal Growth, Surfaces and Applications. European Materials Research Society Monographs. Vol. 1. 2010, Elsevier Ltd. 296.

30. Bolotnikov, A.E., J. Butcher, G.S. Camarda, Y. Cui, S.U. Egarievwe, P. Fochuk, R. Gul, M. Hamade, A. Hossain, K. Kim, O.V. Kopach, M. Petryk, B. Raghothamachar, G. Yang, and R.B. James. Effects of the networks of subgrain boundaries on spectral responses of thick CdZnTe detectors. in Hard X-Ray, Gamma-Ray, and Neutron Detector Physics XIII, August 22, 2011 August 24, 2011. 2011. San Diego, CA, United states: SPIE.

31. Xu, L., A.E. Bolotnikov, A. Hossain, K.H. Kim, R. Gul, G. Yang, G.S. Camarda, L. Marchini, Y. Cui, R.B. James, Y. Xu, T. Wang, and W. Jie. Extended defects in as-grown CdZnTe. in Hard XRay, Gamma-Ray, and Neutron Detector Physics XII, August 2, 2010 - August 4, 2010. 2010. San Diego, CA, United states: SPIE.

32. Yang, G., A.E. Bolotnikov, G.S. Camarda, Y. Cui, A. Hossain, K. Kim, and R.B. James. Electric field distribution of cadmium zinc telluride (CZT) detectors. in Hard X-Ray, Gamma-Ray, and Neutron Detector Physics XI, August 3, 2009 - August 6, 2009. 2009. San Diego, CA, United states: SPIE.

33. Carini, G.A., A.E. Bolotnikov, G.S. Camarda, and R.B. James, "High-resolution X-ray mapping of CdZnTe detectors," Nuclear Instruments and Methods in Physics Research, Section A: Accelerators, Spectrometers, Detectors and Associated Equipment, 2007, 579(1), 120-124.

34. Bolotnikov, A.E., G.S. Camarda, G.A. Carini, Y. Cui, L. Li, and R.B. James, "Cumulative effects of Te precipitates in CdZnTe radiation detectors," Nuclear Instruments and Methods in Physics Research, Section A: Accelerators, Spectrometers, Detectors and Associated Equipment, 2007, 571(3), 687-698.

35. Bolotnikov, A.E., G.S. Camarda, G.A. Carini, Y. Cui, K.T. Kohman, L. Li, M.B. Salomon, and R.B. James, "Performance-limiting defects in CdZnTe detectors," IEEE Transactions on Nuclear Science, 2007, 54(4), 821-827.

36. Carini, G.A., A.E. Bolotnikov, G.S. Camarda, G.W. Wright, R.B. James, and L. Li, "Effect of Te precipitates on the performance of CdZnTe detectors," Applied Physics Letters, 2006, 88(14), 143515.

37. Bolotnikov, A.E., M. Black, G.S. Camarda, G.A. Carini, Y. Cui, K.T. Kohman, L. Li, M.B. Salomon, and R.B. James. The effect of Te precipitates on characteristics of CdZnTe detectors. in Hard X-Ray and Gamma-Ray Detector Physics and Penetrating Radiation Systems VIII. 2006. Bellingham WA, WA 98227-0010, United States: International Society for Optical Engineering.

38. Camarda, G.S., G. Carini, Z. Zhong, D.P. Siddons, A.E. Bolotnikov, G.W. Wright, and R.B. James. Study of growth defects in CZT and their influence on detector uniformity. in Hard X-Ray and Gamma-Ray Detector Physics VI, August 2, 2004 - August 3, 2004. 2004. Denver, CO, United states: SPIE. 
39. Rudolph, P., "Non-stoichiometry related defects at the melt growth of semiconductor compound crystals - A review," Crystal Research and Technology, 2003, 38(7-8), 542-554.

40. Gasperino, D., M. Bliss, K. Jones, K. Lynn, and J.J. Derby, "On crucible effects during the growth of cadmium zinc telluride in an electrodynamic gradient freeze furnace," Journal of Crystal Growth, 2009, 311(8), 2327-35.

41. Miller, W., "Numerical simulations of bulk crystal growth on different scales: silicon and GeSi," Physica Status Solidi B, 2010, 247(4), 855-69.

42. Muller, G. and J. Friedrich. Challenges in modeling of bulk crystal growth. in Proceedings of the Fourth International Workshop on Modeling, November 4, 2003 - November 7, 2003. 2004.

Kyushu, Japan: Elsevier.

43. Sinno, T., E. Dornberger, W. von Ammon, R.A. Brown, and F. Dupret, "Defect engineering of Czochralski single-crystal silicon," Materials Science and Engineering: R: Reports, 2000, 28(5), 149.

44. Batra, R.C., Elements of Continuum Mechanics. 2006: VA: AIAA.

45. Eringen, A., Mechanics of Continua. 1980: Krieger Pub Co.

46. Q. Xiao, S.K., A. Yeckel, J. J. Derby, "On the effects of ampoule tilting during vertical Bridgman growth: three-dimensional computations via a massively parallel finite element method," Journal of Crystal Growth, 1996, 167, 12.

47. H. Lee, A.J.P., "Simulation of radial dopant segregation in vertical Bridgman growth of Gase, a semiconductor with anisotropic solid-phase thermal conductivity," Journal of Crystal Growth, 2001, 231, 22.

48. T. Inamuro, M.Y., H. Inoue, R. Mizuno, F. Ogino, "A lattice Boltzmann method for a binary miscible fluid mixture and its application to a heat-transfer problem," J. Comput. Phys., 2002, 179, 14 .

49. M.C. Sukop, D.T.T., Lattice Boltzmann modeling: An Introduction for Geoscientists and Engineers, first ed. 2005: Springer.

50. Luo, L.-S. The lattice-gas and lattice Boltzmann method: past, present, and future. in International Conference on Applied Computational Fluid Dynamics. 2000. Beijing, China.

51. Hoffman, J.D., Numerical Methods for Engineers and Scientists. Second ed. 1992, New York: McGraw-Hill, Inc.

52. A. Ecer, J.P., N. Satofuka, S. Taylor, Parallel Computational Fluid Dynamics. Implementations and Results Using Parallel Computers. 1996: Elsevier Science B. V.

53. Inamuro, T., "A lattice kinetic scheme for incompressible viscous flows with heat transfer," Philos. T. Roy. Soc, 2002, A 360, 7.

54. A. Yeckel, J.J.D., "Effect of accelerated crucible rotation on melt composition in high-pressure vertical Bridgman growth of cadmium zinc telluride," Journal of Crystal Growth, 2000, 209, 16.

55. A.R. Wadhwa, V.M., J. Abraham. Numerical studies of droplet interactions. in International Conference on Liquid Atomization and Spray Systems. 2003. Sorrento, Italy.

56. G. Tryggvason, B.B., A. Esmaeeli, D. Juric, N. Al-Rawahi, W. Tauber, J. Han, S. Nas, Y.J. Jan, "A front-tracking method for the computations of multiphase flow," J. Comput. Phys., 2001, 162, 51.

57. M. Yildiz, S.D., "A continuum model for the Liquid Phase Diffusion growth of bulk SiGe single crystals," International Journal of Engineering Science, 2005, 43, 21.

58. H. Lee, A.J.P., "Interface Shape and Thermally-Driven Convection in Vertical Bridgman Growth of Gallium Selenide: A Semiconductor With Anisotropic Solid-Phase Thermal Conductivity," Journal of Heat Transfer, 2001, 123, 12.

59. Daly, B.J., "Numerical study of the effect of surface tension on interface instability," Phys. Fluids, 1969, 12, 14.

60. C.W. Hirt, B.D.N., "Volume of fluid (VOF) method for the dynamics of free boundaries," J. Comput. Phys., 1981, 39, 24. 
61. Sethian, J.A., Level Set Methods: Evolving Interfaces in Geometry, Fluid Mechanics, Computer Vision, and Materials Science. 1996: Cambridge University Press.

62. W. J. Boettinger, J.A.W., C. Beckermann, and A. Karma, "Phase-Field Simulation of Solidifcation," Annual Review of Materials Research, 2002, 32, 31.

63. Fix, G., Free Boundary Problems: Theory and Applications. 1983, Piman, Boston.

64. B. Echebarria, R.F., A. Karma, M. Plapp, "Quantitative phase-field model of alloy solidification," Physical review E, 2004, 70, 12.

65. A. Karma, W.-J.R., "Quantitative phase-field modeling of dendritic growth in two and three dimensions," Physical review E, 1998, 57(4), 26.

66. Z. Xu, P.M., "Phase-field modeling of solute precipitation and dissolution," J. Chemical Physics, 2008, 129, 014705.

67. Z. Xu, P.M., "Phase-field modeling of two-dimensional solute precipitation: radial solid fingers and diffusion-limited aggregation," J. Chemical Physics, 2011, 134, 044137.

68. D. Medvedev, K.K., "Lattice-Boltzmann scheme for dendritic growth in presence of convection," Journal of Crystal Growth, 2005, 275(1-2), 5.

69. Chatterjee, D., "Lattice Boltzmann Simulation of Incompressible Transport Phenomena in Macroscopic Solidification Processes," Numerical Heat Transfer, 2010, 58(1), 17.

70. J. Bao, L.S., "Lattice Boltzmann equation model for multi-component multi-phase flow with high density ratios," Applied Mathematical Modelling, 2012, In press, available online 9 May 2012.

71. X. Shan, D.D., "Diffusion in a multicomponent lattice Boltzmann equation model," Phys. Rev. E, 1996, 54, 6.

72. X. Shan, G.D.D., "Multicomponent lattice-Boltzmann model with interparticle interaction," J. Stat. Phys., 1995, 81, 14.

73. X. Shan, H.C., "Lattice Boltzmann model for simulation flows with multiple phases and components," Phys. Rev. E, 1993, 47, 4.

74. X. Shan, H.C., "Simulation of nonideal gases and liquid-gas phase transitions by the lattice Boltzmann equation," Phys. Rev. E, 1994, 49, 7.

75. M. Swift, S.O., W. Osborn, J. Yeomans, "Lattice Boltzmann simulations of liquid-gas and binary-fluid systems," Phys. Rev. E, 1996, 54, 11.

76. M. Swift, W.O., J. Yeomans, "Lattice Boltzmann simulation of nonideal fluids," Phys. Rev. Lett., 1995, 75, 3.

77. P. Capper, J.C.B., C. L. Jones, W. G. Coates, J. J. G. Gosney, C. Ard, I. Kenworthy, "Interfaces and flow regimes in ACRT Grown Cd Hg Te Crystals," Journal of Crystal Growth, 1988, 89, 5.

78. P. CAPPER, J.J.G.G.a.C.L.J., "Application of the Accelerated Crucible Rotation Technique to the Bridgman Growth of Cd Hg Te: Simulations and Crystal Growth," Journal of Crystal Growth, 1984, 70, 8.

79. P. CApper, W.G.C., C. L. Jones, J. J. Gosney, C. K. Ard I. Kenworthy, "Quenching Studies in Cd Hg Te Crystals Grown Using ACRT," Journal of Crystal Growth, 1987, 83, 7.

80. W. G. Coater, P.C., C. L. Jones, J. J. G. Gosney, C. K. Ard, I. Kenworthy, A. Clark, "Effect of ACRT rotation parameters on Bridgman Grown Cd Hg Te Crystals," Journal of Crystal Growth, 1989, 94, 7.

81. Schulz-Dubois, E.O., "Accelerated crucible rotation: hydrodynamics and stirring effect," Journal of Crystal Growth, 1972, 12, 6.

82. G. P. Neitzel, S.H.D., "Centrifugal instabilities during spin-down to rest in finite cylinders. Numerical experiments," Journal of Fluid Mechanics, 1981, 102, 23.

83. W.G. Coates, P.C., C.L. Jones, J.J.G. Gosney, C.K. Ard, I. Kenworthy, A. Clark, "Effect of ACRT rotation parameters on Bridgman grown CdxHg1-xTe crystals," Journal of Crystal Growth, 1989, 94(4), 7.

84. K. Mazuruk, N.R., M. P. Volz. Traveling Magnetic Field Induced Flow for Crystal Growth Applications. in The 37th AIAA Aerospace Sciences Meeting. 1999. Reno, NV, USA. 
85. R. N. Grugel, K.M. Mixing Dynamics Induced by Traveling Magnetic Fields. in The 39th AIAA Aerospace Sciences Meeting. 2001. Reno, NV, USA.

86. S. Yesilyurt, S.M., R. Grugel, K. Mazuruk, "The effect of the traveling magnetic field (TMF) on the buoyancy-induced convection in the vertical Bridgman growth of semiconductors," Journal of Crystal Growth, 2004, 263, 9.

87. P. Dold, K.W.B., "Rotating Magnetic Fields: Fluid Flow and Crystal Growth Applciations," Progress in Crystal Growth and Characterization of Materials, 1999, 31.

88. Szeles, C., S.E. Cameron, J.O. Ndap, and W.C. Chalmers, "Advances in the crystal growth of semi-insulating CdZnTe for radiation detector applications," Ieee Transactions on Nuclear Science, 2002, 49(5), 2535-2540.

89. Fujiwara, K., "Crystal Growth Behaviors of Silicon during Melt Growth Processes," International Journal of Photoenergy, 2012.

90. Hurle, D.T.J. and P. Rudolph, "A brief history of defect formation, segregation, faceting, and twinning in melt-grown semiconductors," Journal of Crystal Growth, 2004, 264(4), 550-564.

91. Rudolph, P., "Fundamental-Studies on Bridgman Growth of Cdte," Progress in Crystal Growth and Characterization of Materials, 1994, 29(1-4), 275-381.

92. Jackson, K.A., "Crystal-Growth Kinetics," Materials Science and Engineering, 1984, 65(1), 7-13.

93. Jackson, K.A., "Interface Structure and Crystal-Growth Kinetics," Abstracts of Papers of the American Chemical Society, 1980, 179(Mar), 72-Coll.

94. Landman, U., W.D. Luedtke, R.N. Barnett, C.L. Cleveland, M.W. Ribarsky, E. Arnold, S. Ramesh, H. Baumgart, A. Martinez, and B. Khan, "Faceting at the Silicon (100) Crystal-Melt Interface - Theory and Experiment," Physical Review Letters, 1986, 56(2), 155-158.

95. Abraham, F.F. and J.Q. Broughton, "Pulsed Melting of Silicon (111) and (100) Surfaces Simulated by Molecular-Dynamics," Physical Review Letters, 1986, 56(7), 734-737.

96. Bales, G.S. and A. Zangwill, "Morphological Instability of a Terrace Edge during Step-Flow Growth," Physical Review B, 1990, 41(9), 5500-5508.

97. Nishizawa, H., F. Hori, and R. Oshima, "In-situ HRTEM observation of the meltingcrystallization process of silicon," Journal of Crystal Growth, 2002, 236(1-3), 51-58.

98. Buta, D., M. Asta, and J.J. Hoyt, "Kinetic coefficient of steps at the Si(111) crystal-melt interface from molecular dynamics simulations," Journal of Chemical Physics, 2007, 127(7).

99. Aziz, M.J. and C.W. White, "Solute Trapping in Silicon by Lateral Motion of (111) Ledges," Physical Review Letters, 1986, 57(21), 2675-2678.

100. Aziz, M.J. and T. Kaplan, "Continuous Growth-Model for Interface Motion during Alloy Solidification," Acta Metallurgica, 1988, 36(8), 2335-2347.

101. Aziz, M.J., "Interface attachment kinetics in alloy solidification," Metallurgical and Materials Transactions a-Physical Metallurgy and Materials Science, 1996, 27(3), 671-686.

102. Chernov, A.A., S.R. Coriell, and B.T. Murray, "Morphological Stability of a Vicinal Face Induced by Step Flow," Journal of Crystal Growth, 1993, 132(3-4), 405-413.

103. Coriell, S.R., B.T. Murray, A.A. Chernov, and G.B. McFadden, "Step bunching on a vicinal face of a crystal growing in a flowing solution," Journal of Crystal Growth, 1996, 169(4), 773-785.

104. Homma, Y., P. Finnie, and M. Uwaha, "Morphological instability of atomic steps observed on Si(111) surfaces," Surface Science, 2001, 492(1-2), 125-136.

105. Tokairin, M., K. Fujiwara, K. Kutsukake, N. Usami, and K. Nakajima, "Formation mechanism of a faceted interface: In situ observation of the $\mathrm{Si}(100)$ crystal-melt interface during crystal growth," Physical Review B, 2009, 80(17).

106. Fujiwara, K., R. Gotoh, X.B. Yang, H. Koizumi, J. Nozawa, and S. Uda, "Morphological transformation of a crystal-melt interface during unidirectional growth of silicon," Acta Materialia, 2011, 59(11), 4700-4708.

107. Chang, C.J. and R.A. Brown, "Radial Segregation Induced by Natural-Convection and Melt Solid Interface Shape in Vertical Bridgman Growth," Journal of Crystal Growth, 1983, 63(2), 343-364. 
108. Dost, S., Y. Liu, B. Lent, and R.F. Redden, "A numerical simulation study for the effect of applied magnetic field in growth of CdTe single crystals by the traveling heater method," International Journal of Applied Electromagnetics and Mechanics, 2003, 17(4), 271-288.

109. Ghaddar, C.K., C.K. Lee, S. Motakef, and D.C. Gillies, "Numerical simulation of THM growth of CdTe in presence of rotating magnetic fields (RMF)," Journal of Crystal Growth, 1999, 205(1-2), 97-111.

110. Liu, Y., S. Dost, B. Lent, and R.F. Redden, "A three-dimensional numerical simulation model for the growth of CdTe single crystals by the travelling heater method under magnetic field," Journal of Crystal Growth, 2003, 254(3-4), 285-297.

111. Lemaignan, C. and Y. Malmejac, "Unidirectional Solidification of Irregular Silver-Germanium Eutectic Alloys," Journal of Crystal Growth, 1979, 46(6), 771-778.

112. BenHadid, H., D. Henry, and R. Touihri, "Unsteady three-dimensional buoyancy-driven convection in a circular cylindrical cavity and its damping by magnetic field," Journal of Crystal Growth, 1997, 180(3-4), 433-441.

113. Roux, B., H. Benhadid, and P. Laure, "Numerical-Simulation of Oscillatory Convection in Semiconductor Melts," Journal of Crystal Growth, 1989, 97(1), 201-216.

114. Grasza, K. and M. Pawlowska, "Morphological instabilities in CdTe crystal growth from the vapor phase," Journal of Crystal Growth, 1999, 203(3), 371-375.

115. Roy, U.N., S. Weiler, and J. Stein, "Growth and interface study of 2 in diameter CdZnTe by THM technique," Journal of Crystal Growth, 2010, 312(19), 2840-2845.

116. Greenberg, J.H., V.N. Guskov, V.B. Lazarev, and O.V. Shebershneva, "Vapor-Pressure Scanning of Nonstoichiometry in Cdte," Journal of Solid State Chemistry, 1993, 102(2), 382-389.

117. Heffelfinger, J.R., D.L. Medlin, and R.B. James, "Analysis of grain boundaries, twin boundaries and Te precipitates in Cd1-xZnxTe grown by high-pressure Bridgman method," Semiconductors for Room-Temperature Radiation Detector Applications Ii, 1997, 487, 33-38.

118. Heffelfinger, J.R., D.L. Medlin, and R.B. James, "Structural and chemical analysis of grain boundaries and tellurium precipitates in commercial Cd1-xZnxTe," Hard X-Ray and Gamma-Ray Detector Physics and Applications, 1998, 3446, 49-54.

119. Heffelfinger, J.R., D.L. Medlin, and R.B. James, "Analysis of grain boundaries, twin boundaries and Te precipitates in Cd1-xZnxTe grown by high-pressure Bridgman method," Infrared Applications of Semiconductors Ii, 1998, 484, 247-252.

120. Heffelfinger, J.R., D.L. Medlin, H. Yoon, H. Hermon, and R.B. James, "Analysis of Cd1-xZnxTe microstructure," Hard X-Ray and Gamma-Ray Detector Physics, Optics, and Applications, 1997, 3115, 40-50.

121. Bardsley, W., D.T.J. Hurle, M. Hart, and A.R. Lang, "Structural and Chemical Inhomogeneities in Germanium Single-Crystals Grown under Conditions of Constitutional Supercooling," Journal of Crystal Growth, 1980, 49(4), 612-630.

122. Naumann, M., P. Rudolph, M. Neubert, and J. Donecker, "Dislocation studies in VCz GaAs by laser scattering tomography," Journal of Crystal Growth, 2001, 231(1-2), 22-30.

123. Schmid, E. and W. Boas, "Plasticity of Crystals," 1968, Chapman \& Hall, London, 1968.

124. Jordan, A.S., A.R. Vonneida, and R. Caruso, "The Theory and Practice of Dislocation Reduction in Gaas and Inp," Journal of Crystal Growth, 1984, 70(1-2), 555-573.

125. Billig, E., "Some Defects in Crystals Grown from the Melt .1. Defects Caused by Thermal Stresses," Proceedings of the Royal Society of London Series a-Mathematical and Physical Sciences, 1956, 235(1200), 37-\&.

126. Indenbom, V.L., "Formation of Stress and Dislocations in Crystal-Growth," Kristall Und Technik-Crystal Research and Technology, 1979, 14(5), 493-507.

127. Hurle, D.T.J., "A Mechanism for Twin Formation during Czochralski and Encapsulated Vertical Bridgman Growth of Iii-V Compound Semiconductors," Journal of Crystal Growth, 1995, 147(34), 239-250. 
128. Sundaram, S.K., C.H. Henager, D.J. Edwards, A.L. Schemer-Kohrn, M. Bliss, B.R. Riley, M.B. Toloczko, and K.G. Lynn, "Hierarchical microstructures in CZT," Nuclear Instruments \& Methods in Physics Research Section a-Accelerators Spectrometers Detectors and Associated Equipment, 2011, 652(1), 174-177.

129. Henager, C.H., D.J. Edwards, A.L. Schemer-Kohrn, S.K. Sundaram, B.J. Riley, and M. Bliss, "Electron backscatter diffraction of a Ge growth tip from a vertical gradient freeze furnace," Journal of Crystal Growth, 2008, 311(1), 10-14.

130. Chung, H., M. Dudley, D.J. Larson, D.T.J. Hurle, D.F. Bliss, and V. Prasad, "The mechanism of growth-twin formation in zincblende crystals: new insights from a study of magnetic liquid encapsulated Czochralski-grown InP single crystals," Journal of Crystal Growth, 1998, 187(1), 917.

131. Dudley, M., B. Raghothamachar, Y. Guo, X.R. Huang, H. Chung, D.J. Larson, D.T.J. Hurle, D.F. Bliss, V. Prasad, and Z. Huang, "The mechanism of twinning in zincblende structure crystals: New insights on polarity effects from a study of magnetic liquid encapsulated Czochralski grown InP single crystals," Applications of Synchrotron Radiation Techniques to Materials Science Iv, 1998, 524, 65-70.

132. Vere, A.W., S. Cole, and D.J. Williams, "The Origins of Twinning in Cdte," Journal of Electronic Materials, 1983, 12(3), 551-561.

133. Milenov, T.I. and M.M. Gospodinov, "Deformational twinning in CdTe crystals grown by the Bridgman method," Materials Science and Engineering B-Solid State Materials for Advanced Technology, 2001, 84(3), 189-194.

134. Fissel, A. and M. Schenk, "Deformation Characteristics and Real Structure around Indents on (111)(a)-Surfaces of Ii-Vi-Solid Solutions," Journal of Materials Science-Materials in Electronics, 1992, 3(3), 147-156.

135. Fujiwara, K., K. Maeda, N. Usami, G. Sazaki, Y. Nose, and K. Nakajima, "Formation mechanism of parallel twins related to Si-facetted dendrite growth," Scripta Materialia, 2007, 57(2), 81-84.

136. Yang, X.B., K. Fujiwara, R. Gotoh, K. Maeda, J. Nozawa, H. Koizumi, and S. Uda, "Effect of twin spacing on the growth velocity of Si faceted dendrites," Applied Physics Letters, 2010, 97(17).

137. Yang, X.B., K. Fujiwara, K. Maeda, J. Nozawa, H. Koizumi, and S. Uda, "Dependence of SiFaceted Dendrite Growth Orientation on Twin Spacing and Undercooling," Crystal Growth \& Design, 2011, 11(4), 1402-1410.

138. Dudley, M., B. Raghothamachar, Y. Guo, X.R. Huang, H. Chung, D.T.J. Hurle, and D.F. Bliss, "The influence of polarity on twinning in zincblende structure crystals: new insights from a study of magnetic liquid encapsulated, Czochralski grown InP single crystals," Journal of Crystal Growth, 1998, 192(1-2), 1-10.

139. R. S. Rai, S.M., D. J. Michel, H. H. Smith, S. McDevitt, C. J. Johnson, "Deformation behavior of CdTe and (Cd,Zn)Te single crystals between 200 and $600{ }^{\circ} \mathrm{C}, " 1991,7$.

140. Winkler, M., M. Schenk, and I. Hahnert, "Deformation Induced Microtwins in Cdte," Crystal Research and Technology, 1992, 27(8), 1047-1051.

141. Hu, S.Y., C.H. Henager, and L.Q. Chen, "Simulations of stress-induced twinning and detwinning: A phase field model," Acta Materialia, 2010, 58(19), 6554-6564.

142. Kibey, S., J.B. Liu, D.D. Johnson, and H. Sehitoglu, "Predicting twinning stress in fcc metals: Linking twin-energy pathways to twin nucleation," Acta Materialia, 2007, 55(20), 6843-6851.

143. Li, B. and E. Ma, "Atomic Shuffling Dominated Mechanism for Deformation Twinning in Magnesium," Physical Review Letters, 2009, 103(3).

144. Kibey, S., J.B. Liu, D.D. Johnson, and H. Sehitoglu, "Energy pathways and directionality in deformation twinning," Applied Physics Letters, 2007, 91(18).

145. Glimm, J., J.W. Grove, X.L. Li, K.M. Shyue, Y.N. Zeng, and Q. Zhang, "Three-dimensional front tracking," Siam Journal on Scientific Computing, 1998, 19(3), 703-727. 
146. Scardovelli, R. and S. Zaleski, "Direct numerical simulation of free-surface and interfacial flow," Annual Review of Fluid Mechanics, 1999, 31, 567-603.

147. Osher, S. and R.P. Fedkiw, "Level set methods: An overview and some recent results," Journal of Computational Physics, 2001, 169(2), 463-502.

148. Cahn, J.W. and J.E. Hilliard, "Free Energy of a Nonuniform System .1. Interfacial Free Energy," Journal of Chemical Physics, 1958, 28(2), 258-267.

149. Allen, S.M. and J.W. Cahn, "Microscopic Theory for Antiphase Boundary Motion and Its Application to Antiphase Domain Coarsening," Acta Metallurgica, 1979, 27(6), 1085-1095.

150. Chen, L.Q., "Phase-field models for microstructure evolution," Annual Review of Materials Research, 2002, 32, 113-140.

151. Cross, M.C. and P.C. Hohenberg, "Pattern-Formation Outside of Equilibrium," Reviews of Modern Physics, 1993, 65(3), 851-1112.

152. Kurz, W. and R. Trivedi, "Solidification Microstructures - Recent Developments and FutureDirections," Acta Metallurgica Et Materialia, 1990, 38(1), 1-17.

153. Boettinger, W.J., S.R. Coriell, A.L. Greer, A. Karma, W. Kurz, M. Rappaz, and R. Trivedi, "Solidification microstructures: Recent developments, future directions," Acta Materialia, 2000, 48(1), 43-70.

154. Echebarria, B., R. Folch, A. Karma, and M. Plapp, "Quantitative phase-field model of alloy solidification," Physical Review E, 2004, 70(6).

155. Kim, S.G., W.T. Kim, and T. Suzuki, "Phase-field model for binary alloys," Physical Review E, 1999, 60(6), 7186-7197.

156. Ofori-Opoku, N. and N. Provatas, "A quantitative multi-phase field model of polycrystalline alloy solidification," Acta Materialia, 2010, 58(6), 2155-2164.

157. Asta, M., C. Beckermann, A. Karma, W. Kurz, R. Napolitano, M. Plapp, G. Purdy, M. Rappaz, and R. Trivedi, "Solidification microstructures and solid-state parallels: Recent developments, future directions," Acta Materialia, 2009, 57(4), 941-971.

158. Broughton, J.Q. and G.H. Gilmer, "Molecular-Dynamics Investigation of the Crystal Fluid Interface .6. Excess Surface Free-Energies of Crystal Liquid-Systems," Journal of Chemical Physics, 1986, 84(10), 5759-5768.

159. Hoyt, J.J., M. Asta, and A. Karma, "Atomistic and continuum modeling of dendritic solidification," Materials Science \& Engineering R-Reports, 2003, 41(6), 121-163.

160. Davidchack, R.L. and B.B. Laird, "Crystal structure and interaction dependence of the crystalmelt interfacial free energy," Physical Review Letters, 2005, 94(8).

161. Laird, B.B. and R.L. Davidchack, "Direct calculation of the crystal-melt interfacial free energy via molecular dynamics computer simulation," Journal of Physical Chemistry B, 2005, 109(38), 17802-17812.

162. Morris, J.R., M.I. Mendelev, and D.J. Srojovitz, "A comparison of crystal-melt interfacial free energies using different Al potentials," Journal of Non-Crystalline Solids, 2007, 353(32-40), 3565-3569.

163. Fehlner, W.R. and S.H. Vosko, "Product Representation for Cubic Harmonics and Special Directions for Determination of Fermi-Surface and Related Properties," Canadian Journal of Physics, 1976, 54(21), 2159-2169.

164. Haxhimali, T., A. Karma, F. Gonzales, and M. Rappaz, "Orientation selection in dendritic evolution," Nature Materials, 2006, 5(8), 660-664.

165. Becker, C.A., D. Olmsted, M. Asta, J.J. Hoyt, and S.M. Foiles, "Atomistic underpinnings for orientation selection in alloy dendritic growth," Physical Review Letters, 2007, 98(12).

166. Hoyt, J.J., B. Sadigh, M. Asta, and S.M. Foiles, "Kinetic phase field parameters for the Cu-Ni system derived from atomistic computations," Acta Materialia, 1999, 47(11), 3181-3187.

167. Mikheev, L.V. and A.A. Chernov, "Mobility of a Diffuse Simple Crystal Melt Interface," Journal of Crystal Growth, 1991, 112(2-3), 591-596. 
168. Huitema, H.E.A., M.J. Vlot, and J.P. van der Eerden, "Simulations of crystal growth from Lennard-Jones melt: Detailed measurements of the interface structure," Journal of Chemical Physics, 1999, 111(10), 4714-4723.

169. Amini, M. and B.B. Laird, "Kinetic coefficient for hard-sphere crystal growth from the melt," Physical Review Letters, 2006, 97(21).

170. ten Wolde, P.R., M.J. Ruiz-Montero, and D. Frenkel, "Numerical calculation of the rate of homogeneous gas-liquid nucleation in a Lennard-Jones system," Journal of Chemical Physics, 1999, 110(3), 1591-1599.

171. Auer, S. and D. Frenkel, "Prediction of absolute crystal-nucleation rate in hard-sphere colloids," Nature, 2001, 409(6823), 1020-1023.

172. Desgranges, C. and J. Delhommelle, "Molecular simulation of the crystallization of aluminum from the supercooled liquid," Journal of Chemical Physics, 2007, 127(14).

173. Aga, R.S., J.R. Morris, J.J. Hoyt, and M. Mendelev, "Quantitative parameter-free prediction of simulated crystal-nucleation times," Physical Review Letters, 2006, 96(24).

174. Wu, D.T., L. Granasy, and F. Spaepen, "Nucleation and the solid-liquid interfacial free energy," Mrs Bulletin, 2004, 29(12), 945-950.

175. Gasser, U., E.R. Weeks, A. Schofield, P.N. Pusey, and D.A. Weitz, "Real-space imaging of nucleation and growth in colloidal crystallization," Science, 2001, 292(5515), 258-262.

176. Miller, W., I. Rasin, and S. Succi, "Lattice Boltzmann phase-field modelling of binary-alloy solidification," Physica a-Statistical Mechanics and Its Applications, 2006, 362(1), 78-83.

177. Medvedev, D. and K. Kassner, "Lattice Boltzmann scheme for crystal growth in external flows," Physical Review E, 2005, 72(5).

178. Miller, W., F. Pimentel, I. Rasin, and U. Rehse, "3D parallel calculations of dendritic growth with the lattice Boltzmann method," Parallel Computing: Software Technology, Algorithms, Architectures and Applications, 2004, 13, 291-296.

179. Wheeler, A.A., "Phase-field theory of edges in an anisotropic crystal," Proceedings of the Royal Society a-Mathematical Physical and Engineering Sciences, 2006, 462(2075), 3363-3384.

180. Wise, S., J. Kim, and J. Lowengrub, "Solving the regularized, strongly anisotropic Cahn-Hilliard equation by an adaptive nonlinear multigrid method," Journal of Computational Physics, 2007, 226(1), 414-446.

181. Ratz, A. and A. Voigt, "Higher order regularization of anisotropic geometric evolution equations in three dimensions," Journal of Computational and Theoretical Nanoscience, 2006, 3(4), 560 564.

182. Jamet, D. and C. Misbah, "Toward a thermodynamically consistent picture of the phase-field model of vesicles: Curvature energy," Physical Review E, 2008, 78(3).

183. Torabi, S., J. Lowengrub, A. Voigt, and S. Wise, "A new phase-field model for strongly anisotropic systems," Proceedings of the Royal Society a-Mathematical Physical and Engineering Sciences, 2009, 465(2105), 1337-1359.

184. Wang, Y.U., Y.M. Jin, A.M. Cuitino, and A.G. Khachaturyan, "Phase field microelasticity theory and modeling of multiple dislocation dynamics," Applied Physics Letters, 2001, 78(16), 23242326.

185. Hu, S.Y., Y.L. Li, Y.X. Zheng, and L.Q. Chen, "Effect of solutes on dislocation motion - a phasefield simulation," International Journal of Plasticity, 2004, 20(3), 403-425.

186. Granasy, L., T. Borzsonyi, and T. Pusztai, "Nucleation and bulk crystallization in binary phase field theory," Physical Review Letters, 2002, 88(20).

187. Zhang, L., L.Q. Chen, and Q. Du, "Diffuse-interface approach to predicting morphologies of critical nucleus and equilibrium structure for cubic to tetragonal transformations," Journal of Computational Physics, 2010, 229(18), 6574-6584.

188. Toth, G.I. and L. Granasy, "Phase field theory of interfaces and crystal nucleation in a eutectic system of fcc structure: I. Transitions in the one-phase liquid region," Journal of Chemical Physics, 2007, 127(7). 
189. Toth, G.I. and L. Granasy, "Phase field theory of interfaces and crystal nucleation in a eutectic system of fcc structure: II. Nucleation in the metastable liquid immiscibility region," Journal of Chemical Physics, 2007, 127(7).

190. Simmons, J.P., C. Shen, and Y. Wang, "Phase field modeling of simultaneous nucleation and growth by explicitly incorporating nucleation events," Scripta Materialia, 2000, 43(10), 935-942.

191. Warren, J.A., T. Pusztai, L. Kornyei, and L. Granasy, "Phase field approach to heterogeneous crystal nucleation in alloys," Physical Review B, 2009, 79(1).

192. Bhatnagar, P.L., E.P. Gross, and M. Krook, "A model for collision processes in gases. I. Small amplitude processes in charged and neutral one-component systems," Physical Review, 1954, 94, 511-525.

193. Wheeler, A.A., W.J. Boettinger, and G.B. Mcfadden, "Phase-Field Model of Solute Trapping during Solidification," Physical Review E, 1993, 47(3), 1893-1909.

194. Mullis, A.M., J. Rosam, and P.K. Jimack, "Solute trapping and the effects of anti-trapping currents on phase-field models of coupled thermo-solutal solidification," Journal of Crystal Growth, 2010, 312(11), 1891-1897.

195. Wang, H.F., F. Liu, Z. Chen, and W. Yang, "Solute trapping model based on solute drag treatment," Transactions of Nonferrous Metals Society of China, 2010, 20(5), 877-881.

196. Wang, J.C., J.J. Li, Y.J. Yang, Y.X. Zhang, and G.C. Yang, "Phase field simulation of the interface morphology evolution and its stability during directional solidification of binary alloys," Science in China Series E-Technological Sciences, 2008, 51(4), 362-370.

197. Kauerauf, B., G. Zimmermann, S. Rex, B. Billia, H. Jamgotchian, and J.D. Hunt, "Directional cellular growth of succinonitrile- $0.075 \mathrm{wt} \%$ acetone bulk samples Part 2: Analysis of cellular pattern," Journal of Crystal Growth, 2001, 223(1-2), 277-284.

198. Greenwood, M., M. Haataja, and N. Provatas, "Crossover scaling of wavelength selection in directional solidification of binary alloys," Physical Review Letters, 2004, 93(24).

199. Miller, W. and I. Rasin, "Growth kinetics by means of phase-field methods in applied crystal growth," Journal of Crystal Growth, 2007, 303(1), 95-99.

200. Uehara, T. and R.F. Sekerka, "Phase field simulations of faceted growth for strong anisotropy of kinetic coefficient," Journal of Crystal Growth, 2003, 254(1-2), 251-261.

201. Debierre, J.M., A. Karma, F. Celestini, and R. Guerin, "Phase-field approach for faceted solidification," Physical Review E, 2003, 68(4).

202. Russo, G. and P. Smereka, "A level-set method for the evolution of faceted crystals," Siam Journal on Scientific Computing, 2000, 21(6), 2073-2095.

203. Wang, J.C. and Y. Inatomi, "Three-dimensional Phase Field Modeling of the Faceted Cellular Growth," Isij International, 2010, 50(12), 1901-1907.

204. Chen, P., Y.L. Tsai, and C.W. Lan, "Phase field modeling of growth competition of silicon grains," Acta Materialia, 2008, 56(15), 4114-4122.

205. Salgado-Ordorica, M.A., J.L. Desbiolles, and M. Rappaz, "Study of the twinned dendrite tip shape I: Phase-field modeling," Acta Materialia, 2011, 59(13), 5074-5084.

206. Hu, S.Y. and C.H. Henager, "Phase-field modeling of twin nucleation and evoltuion during CdTe growth," Personal data, 2013.

207. Ratsch, C., M.F. Gyure, R.E. Caflisch, F. Gibou, M. Petersen, M. Kang, J. Garcia, and D.D. Vvedensky, "Level-set method for island dynamics in epitaxial growth," Physical Review B, 2002, 65(19).

208. Sun, D.K., M.F. Zhu, S.Y. Pan, and D. Raabe, "Lattice Boltzmann modeling of dendritic growth in a forced melt convection," Acta Materialia, 2009, 57(6), 1755-1767.

209. Yin, H., S.D. Felicelli, and L. Wang, "Simulation of a dendritic microstructure with the lattice Boltzmann and cellular automaton methods," Acta Materialia, 2011, 59(8), 3124-3136.

210. Tan, L.J. and N. Zabaras, "A level set simulation of dendritic solidification of multi-component alloys," Journal of Computational Physics, 2007, 221(1), 9-40. 
211. Ratz, A. and A. Voigt, "Various phase-field approximations for Epitaxial Growth," Journal of Crystal Growth, 2004, 266(1-3), 278-282.

212. Hu, S.Y. and C.H. Henager, "Phase-field simulations of Te-precipitate morphology and evolution kinetics in Te-rich CdTe crystals," Journal of Crystal Growth, 2009, 311(11), 3184-3194.

213. Volk1, J. and G. Muller, "A New Model for the Calculation of Dislocation Formation in Semiconductor Melt Growth by Taking into Account the Dynamics of Plastic-Deformation," Journal of Crystal Growth, 1989, 97(1), 136-145.

214. Moosbrugger, J.C. and A. Levy, "Constitutive modeling for CdTe single crystals," Metallurgical and Materials Transactions A: Physical Metallurgy and Materials Science, 1995, 26 A(10), 26872697.

215. Moosbrugger, J.C., "Continuum slip viscoplasticity with the Haasen constitutive model: application to CdTe single crystal inelasticity," International Journal of Plasticity, 1995, 11(7), 799-826.

216. Durose, K., Extended Defects in CdTe, in CdTe and Related Compounds; Physics, Defects, Hetero- and Nano-structures, Crystal Growth, Surfaces and Applications, R. Triboulet and P. Siffert, Editors. 2010, Elsevier Ltd. p. 171-227.

217. Gutmanas, E.Y. and P. Haasen, "Thermal activation analysis of dislocation motion in CdTe," Physica Status Solidi A, 1981, 63(1), 193-202.

218. Gutmanas, E.Y., N. Travitzky, U. Plitt, and P. Haasen, "The mechanical behavior of CdTe," Scripta Metallurgica, 1979, 13(4), 293-7.

219. Kubin, L., B. Devincre, and T. Hoc, "Modeling dislocation storage rates and mean free paths in face-centered cubic crystals," Acta Materialia, 2008, 56(20), 6040-9.

220. Devincre, B., T. Hoc, and L. Kubin, "Dislocation mean free paths and strain hardening of crystals," Science, 2008, 320(5884), 1745-8.

221. Gomez-Garcia, D., B. Devincre, and L.P. Kubin, "Dislocation patterns and the similitude principle: 2.5D mesoscale Simulations," Physical Review Letters, 2006, 96(12), 125503-1.

222. Madec, R., B. Devincre, and L.P. Kubin, "Simulation of dislocation patterns in multislip," Scripta Materialia, 2002, 47(10), 689-95.

223. Kubin, L.P., B. Devincre, and M. Tang, "Mesoscopic modelling and simulation of plasticity in fcc and bec crystals: Dislocation intersections and mobility," Journal of Computer-Aided Materials Design, 1998, 5(1), 31-54.

224. Bulatov, V.V. and L.P. Kubin, "Dislocation modelling at atomistic and mesoscopic scales," Current Opinion in Solid State \&amp; Materials Science, 1998, 3(6), 558-61.

225. Bulatov, V., F.F. Abraham, L. Kubin, B. Devincre, and S. Yip, "Connecting atomistic and mesoscale simulations of crystal plasticity," Nature, 1998, 391(6668), 669-72.

226. Kubin, L.P., "Dislocation patterning during multiple slip of FCC crystals. A simulation approach," Physica Status Solidi A, 1993, 135(2), 433-43.

227. Kubin, L.P., G. Canova, M. Condat, B. Devincre, V. Pontikis, and Y. Brechet, "Dislocation microstructures and plastic flow: a 3D simulation," Diffusion and Defect Data - Solid State Data, Part B (Solid State Phenomena), 1992, 23-24, 455-72.

228. Devincre, B. and M. Condat, "Model validation of a 3D simulation of dislocation dynamics: discretization and line tension effects," Acta metallurgica et materialia, 1992, 40(10), 2629-2637.

229. Ispnovity, P.D., I. Groma, W. Hoffelner, and M. Samaras, "Abnormal subgrain growth in a dislocation-based model of recovery," Modelling and Simulation in Materials Science and Engineering, 2011, 19(4).

230. Du, M.H., H. Takenaka, and D.J. Singh, "Native defects and oxygen and hydrogen-related defect complexes in CdTe: Density functional calculations," Journal of Applied Physics, 2008, 104(9).

231. Dellago, C. and P.G. Bolhuis, "Transition Path Sampling and Other Advanced Simulation Techniques for Rare Events," Adv. Polym. Sci., 2008.

232. Langer, T., A. Kruse, F.A. Ketzer, A. Schwiegel, L. Hoffmann, H. Jonen, H. Bremers, U. Rossow, and A. Hangleiter, "Origin of the "green gap": Increasing nonradiative recombination in 
indium-rich GaInN/GaN quantum well structures," Physica Status Solidi (C) Current Topics in Solid State Physics, 2011, 8(7-8), 2170-2172.

233. Omling, P., E.R. Weber, L. Montelius, H. Alexander, and J. Michel, "Electrical properties of dislocations and point defects in plastically deformed silicon," Physical Review B (Condensed Matter), 1985, 32(10), 6571-81.

234. Wessel, K. and H. Alexander, "On the mobility of partial dislocations in silicon," Philosophical Magazine, 1977, 35(6), 1523-36.

235. Norskov, J.K. and N.D. Lang, "Effective-medium theory of chemical binding: Application to chemisorption," Physical Review B (Condensed Matter), 1980, 21(6), 2131-6.

236. Finnis, M.W. and J.E. Sinclair, "A simple empirical N-body potential for transition metals," Philosophical Magazine A (Physics of Condensed Matter, Defects and Mechanical Properties), 1984, 50(1), 45-55.

237. Daw, M.S. and M.I. Baskes, "Embedded-atom method: derivation and application to impurities, surfaces, and other defects in metals," Physical Review B (Condensed Matter), 1984, 29(12), 6443-53.

238. Tersoff, J., "New empirical approach for the structure and energy of covalent systems," Physical Review B (Condensed Matter), 1988, 37(12), 6991-7000.

239. Minervini, L., R.W. Grimes, and K.E. Sickafus, "Disorder in pyrochlore oxides," Journal of the American Ceramic Society, 2000, 83(8), 1873-8.

240. Valone, S.M. and S.R. Atlas, "An empirical charge redistribution model for oxide fuel materials," Transactions of the American Nuclear Society, 2005, 92, 713-14.

241. Valone, S.M. and S.R. Atlas, "An empirical charge transfer potential with correct dissociation limits," Journal of Chemical Physics, 2004, 120(16), 7262-73.

242. Daw, M.S., S.M. Foiles, and M.I. Baskes, "The embedded-atom method: a review of theory and applications," Material Science Reports, 1993, 9(7-8), 251-310.

243. Ackland, G.J. and V. Vitek, "Many-body potentials and atomic-scale relaxations in noble-metal alloys," Physical Review B (Condensed Matter), 1990, 41(15), 10324-33.

244. Ackland, G.J., M.W. Finnis, and V. Vitek, "Validity of the second moment tight-binding model," Journal of Physics F (Metal Physics), 1988, 18(8), 153-7.

245. Ercolessi, F., E. Tosatti, and M. Parinello, "Au(100) surface reconstruction," Physical Review Letters, 1986, 57(6), 719-22.

246. Ercolessi, F., M. Parrinello, and E. Tosatti, "Au(100) reconstruction in the glue model," Surface Science, 1986, 177(2), 314-28.

247. Sutton, A.P. and J. Chen, "Long-range Finnis-Sinclair potentials," Philosophical Magazine Letters, 1990, 61(3), 139-46.

248. Nelson, J.S., M.S. Daw, and E.C. Sowa, "Cu(111) and $\mathrm{Ag}(111)$ surface-phonon spectrum: the importance of avoided crossings," Physical Review B (Condensed Matter), 1989, 40(3), 1465-80.

249. Chandra, N. and P. Dang, "Atomistic simulation of grain boundary sliding and migration," Journal of Materials Science, 1999, 34(4), 655-666.

250. Foiles, S.M., M.I. Baskes, and M.S. Daw, "Embedded-atom-method functions for the FCC metals $\mathrm{Cu}, \mathrm{Ag}, \mathrm{Au}, \mathrm{Ni}, \mathrm{Pd}, \mathrm{Pt}$, and their alloys," Physical Review B (Condensed Matter), 1986, 33(12), 7983-91.

251. Foiles, S.M., "Calculation of the surface segregation of Ni-Cu alloys with the use of the embedded-atom method," Physical Review B (Condensed Matter), 1985, 32(12), 7685-93.

252. Foiles, S.M., M.I. Baskes, and M.S. Daw, "Erratum: Embedded-atom-method functions for the fcc metals Cu, Ag, Au, Ni, Pd, Pt, and their alloys [Phys. Rev. B 33, 7983 (1986)]," Physical Review B (Condensed Matter), 1988, 37(17), 10378.

253. Williams, P.L., Y. Mishin, and J.C. Hamilton, "An embedded-atom potential for the $\mathrm{Cu}-\mathrm{Ag}$ system," Modelling and Simulation in Materials Science and Engineering, 2006, 14(5), 817-33.

254. Milstein, F. and S. Chantasiriwan, "Theoretical study of the response of 12 cubic metals to uniaxial loading," Physical Review B (Condensed Matter), 1998, 58(10), 6006-18. 
255. Chantasiriwan, S. and F. Milstein, "Embedded-atom models of 12 cubic metals incorporating second- and third-order elastic-moduli data," Physical Review B (Condensed Matter), 1998, 58(10), 5996-6005.

256. Baskes, M.I., "Application of the embedded-atom method to covalent materials: a semiempirical potential for silicon," Physical Review Letters, 1987, 59(23), 2666-9.

257. Dongare, A.M., L.V. Zhigilei, A.M. Rajendran, and B. LaMattina, "Interatomic potentials for atomic scale modeling of metal-matrix ceramic particle reinforced nanocomposites," Composites Part B: Engineering, 2009, 40(6), 461-467.

258. Dongare, A.M., M. Neurock, and L.V. Zhigilei, "Angular-dependent embedded atom method potential for atomistic simulations of metal-covalent systems," Physical Review B (Condensed Matter and Materials Physics), 2009, 80(18), 184106 (13 pp.).

259. Baskes, M.I., "Modified embedded-atom potentials for cubic materials and impurities," Physical Review B (Condensed Matter), 1992, 46(5), 2727-42.

260. Baskes, M.I. and R.A. Johnson, "Modified embedded atom potentials for HCP metals," Modelling and Simulation in Materials Science and Engineering, 1994, 2(1), 147-63.

261. Baskes, M.I., "Atomistic potentials for the molybdenum-silicon system," Materials Science and Engineering A, 1999, 261(1-2), 165-168.

262. Kuo, C.-L. and P. Clancy, "MEAM molecular dynamics study of a gold thin film on a silicon substrate," Surface Science, 2004, 551(1-2), 39-58.

263. Kuo, C.-L. and P. Clancy, "Development of atomistic MEAM potentials for the silicon-oxygengold ternary system," Modelling and Simulation in Materials Science and Engineering, 2005, 13(8), 1309-29.

264. Yu, J., S.R. Phillpot, and S.B. Sinnott, "Interatomic potential for the structure and energetics of tetrahedrally coordinated silica polymorphs," Physical Review B (Condensed Matter and Materials Physics), 2007, 75(23), 233203-1.

265. Vashishta, P., R.K. Kalia, A. Nakano, and J.P. Rino, "Interaction potentials for alumina and molecular dynamics simulations of amorphous and liquid alumina," Journal of Applied Physics, 2008, 103(8), 083504-1.

266. Vashishta, P., R.K. Kalia, A. Nakano, and J.P. Rino, "Interaction potential for silicon carbide: a molecular dynamics study of elastic constants and vibrational density of states for crystalline and amorphous silicon carbide," Journal of Applied Physics, 2007, 101(10), 103515-1.

267. Pasianot, R., D. Farkas, and E.J. Savino, "Empirical many-body interatomic potential for BCC transition metals," Physical Review B (Condensed Matter), 1991, 43(9), 6952-61.

268. Zhang, B., Y. Ouyang, S. Liao, and Z. Jin, "An analytic MEAM model for all BCC transition metals," Physica B: Condensed Matter, 1999, 262(3, Äi4), 218-225.

269. Lee, B.-J., M.I. Baskes, H. Kim, and Y.K. Cho, "Second nearest-neighbor modified embedded atom method potentials for bcc transition metals," Physical Review B (Condensed Matter and Materials Physics), 2001, 64(18), 184102-1.

270. Kim, H.-K., W.-S. Jung, and B.-J. Lee, "Modified embedded-atom method interatomic potentials for the Fe-Ti-C and Fe-Ti-N ternary systems," Acta Materialia, 2009, 57(11), 3140-3147.

271. Dalgic, S.S., S. Sengul, and S. Kalayc, "The liquid structure of CdTe alloy using the AMEAM potentials," Journal of Optoelectronics and Advanced Materials, 2005, 7(4), 2001-2011.

272. Hu, W., B. Zhang, B. Huang, F. Gao, and D.J. Bacon, "Analytic modified embedded atom potentials for HCP metals," Journal of Physics: Condensed Matter, 2001, 13(6).

273. Hu, W., H. Xu, X. Shu, X. Yuan, B. Gao, and B. Zhang, "Calculation of thermodynamic properties of Mg-RE (RE = Sc, Y, Pr, Nd, Gd, Tb, Dy, Ho or Er)

alloys by an analytic modified embedded atom method," J. Phys. D: Appl. Phys., 2000, 33, 711-718.

274. Hu, W., H. Deng, X. Yuan, and M. Fukumoto, "Point-defect properties in HCP rare earth metals with analytic modified embedded atom potentials," European Physical Journal B, 2003, 34(4), 429-40. 
275. Stillinger, F.H. and T.A. Weber, "Computer simulation of local order in condensed phases of silicon," Physical Review B (Condensed Matter), 1985, 31(8), 5262-71.

276. Ding, K. and H.C. Andersen, "Molecular-dynamics simulation of amorphous germanium," Physical Review B (Condensed Matter), 1986, 34(10), 6987-91.

277. Brenner, D.W., "Empirical potential for hydrocarbons for use in simulating the chemical vapor deposition of diamond films," Physical Review B (Condensed Matter), 1990, 42(15), 9458-71.

278. Roland, C. and G.H. Gilmer, "Binding sites and diffusion barriers of single-height Si(001) steps," Physical Review Letters, 1991, 67(22), 3188-91.

279. Mostoller, M., M.F. Chisholm, and T. Kaplan, "New extended point defect structure in diamond cubic crystals," Physical Review Letters, 1994, 72(10), 1494-7.

280. Mostoller, M., M.F. Chisholm, and T. Kaplan, "Edge-dislocation intersections in diamond cubic crystals," Physical Review B (Condensed Matter), 1994, 50(16), 12183-6.

281. Nandedkar, A.S. and J. Narayan, "Atomic structure of dislocations in silicon, germanium and diamond," Philosophical Magazine A: Physics of Condensed Matter, Structure, Defects and Mechanical Properties, 1990, 61(6), 873-891.

282. Ichimura, M. and J. Narayan, "Role of surface step on misfit dislocation nucleation and critical thickness in semiconductor heterostructures," Materials science \&amp; engineering. B, Solidstate materials for advanced technology, 1995, B31(3), 299-303.

283. Ichimura, M. and J. Narayan, "Atomistic study of dislocation nucleation in $\mathrm{Ge} /(001) \mathrm{Si}$ heterostructures," Philosophical Magazine A (Physics of Condensed Matter, Defects and Mechanical Properties), 1995, 72(2), 281-95.

284. Khor, K.E. and S. Das Sarma, "Equilibrium critical thickness for strained-layer growth," Journal of Vacuum Science \& Technology B: Microelectronics Processing and Phenomena, 1998, 16(4), 2417-2417.

285. Ito, T., K.E. Khor, and S. Das Sarma, "Systematic approach to developing empirical potentials for compound semiconductors," Physical Review B (Condensed Matter), 1990, 41(6), 3893-6.

286. Khor, K.E. and S. Das Sarma, "Proposed universal interatomic potential for elemental tetrahedrally bonded semiconductors," Physical Review B (Condensed Matter), 1988, 38(5), 3318-22.

287. Khor, K.E., T. Ito, and S.D. Sarma, "Empirical interatomic potentials for compound semiconductors: Application to superlattice stability," J. Vac. Sci. Technol. B, 1990, 8, 669.

288. Abell, G.C., "Empirical chemical pseudopotential theory of molecular and metallic bonding," Physical Review B (Condensed Matter), 1985, 31(10), 6184-96.

289. Tersoff, J., "New empirical model for the structural properties of silicon," Physical Review Letters, 1986, 56(6), 632-5.

290. Tersoff, J., "Empirical Interatomic Potential for Carbon, with Applications to Amorphous Carbon," Physical Review Letters, 1988, 61(25), 2879-2882.

291. Tersoff, J., "Modeling solid-state chemistry: interatomic potentials for multicomponent systems," Physical Review B (Condensed Matter), 1989, 39(8), 5566-8.

292. Gao, F. and W.J. Weber, "Empirical potential approach for defect properties in 3C-SiC," Nucl. Instrum. Methods Phys. Res. B, 2002, 191, 504-8.

293. Wang, Z.Q., D. Stroud, and A.J. Markworth, "Monte Carlo study of the liquid CdTe surface," Physical Review B (Condensed Matter), 1989, 40(5), 3129-32.

294. Wang, J. and A. Rockett, "Simulating diffusion on Si(001)21 surfaces using a modified interatomic potential," Physical Review B (Condensed Matter), 1991, 43(15), 12571-9.

295. Henager, C. and J.R. Morris, "Atomistic simulation of CdTe solid-liquid coexistence equilibria," Physical Review B, 2009, 80(Copyright (C) 2010 The American Physical Society), 245309.

296. Ward, D.K., X.W. Zhou, B.M. Wong, F.P. Doty, and J.A. Zimmerman, "Accuracy of existing atomic potentials for the CdTe semiconductor compound," Journal of Chemical Physics, 2011, 134(24). 
297. Zhou, X.W., D.K. Ward, B.M. Wong, F.P. Doty, J.A. Zimmerman, G.N. Nielson, J.L. CruzCampa, V.P. Gupta, J.E. Granata, J.J. Chavez, and D. Zubia, "High-fidelity simulations of CdTe vapor deposition from a bond-order potential-based molecular dynamics method," Physical Review B (Condensed Matter and Materials Physics), 2012, 85(24), 245302 (15 pp.).

298. Ward, D.K., X.W. Zhou, B.M. Wong, F.P. Doty, and J.A. Zimmerman, "Analytical bond-order potential for the cadmium telluride binary system," Physical Review B (Condensed Matter and Materials Physics), 2012, 85(11), 115206 (19 pp.).

299. Hafner, J., "Ab-initio simulations of materials using VASP: Density-functional theory and beyond," Journal of Computational Chemistry, 2008, 29(13), 2044-2078.

300. Kresse, G., J. Furthmuller, and J. Hafner, "Theory of the crystal structures of selenium and tellurium: The effect of generalized-gradient corrections to the local-density approximation," Physical Review B (Condensed Matter), 1994, 50(18), 13181-5.

301. Meixner, M. and E. Scholl, "Kinetically enhanced correlation and anticorrelation effects in selforganized quantum dot stacks," Physical Review B (Condensed Matter and Materials Physics), 2003, 67(12), 121202-1.

302. Schöll, E. and S. Bose, "Kinetic Monte Carlo simulation of the nucleation stage of the selforganized growth of quantum dots," Solid-State Electronics, 1998, 42(7,̈̈ì), 1587-1591.

303. Block, M., R. Kunert, E. Schöll, T. Boeck, and T. Teubner, "Kinetic Monte Carlo simulation of formation of microstructures in liquid droplets," New Journal of Physics, 2004, 6, 166.

304. Boeck, T., T. Teubner, K. Schmidt, and P.-M. Wilde, "Method to grow silicon crystallites on glass," Journal of Crystal Growth, 1999, 198-199(pt 1), 420-424.

305. Martin-Bragado, I. and V. Moroz, "Facet formation during solid phase epitaxy regrowth: a lattice kinetic Monte Carlo model," Applied Physics Letters, 2009, 95(12), 123123 (3 pp.).

306. Chopra, M., M. Muller, and J.J. de Pablo, "Order-parameter-based Monte Carlo simulation of crystallization," Journal of Chemical Physics, 2006, 124(13), 134102-1.

307. Yang, Y., H. Huang, X. S. K., and E. Chason, "Stress control in polycrystalline thin filmsreduction in adatoms diffusion into grain boundaries via surfactants," Applied Physics Letters, 2010, 96(21), 211903 (3 pp.).

308. Moller, C. and M.S. Plesset, "Note on an approximation treatment for many-electron systems," Physical Review, 1934, 46(7), 0618-0622.

309. Polizzi, E., "Density-matrix-based algorithm for solving eigenvalue problems," Physical Review B, 2009, 79(11).

310. Gygi, F., "Large-scale first-principles molecular dynamics: moving from terascale to petascale computing," SciDAC 2006: Scientific Discovery Through Advanced Computing, 2006, 46, 268277.

311. Windus, T.L., E.J. Bylaska, K. Tsemekhman, J. Andzelm, and N. Govind, "Computational Nanoscience with NWChem," Journal of Computational and Theoretical Nanoscience, 2009, 6(6), 1297-1304.

312. Gygi, F., E.W. Draeger, M. Schulz, B.R.d. Supinski, J.A. Gunnels, V. Austel, J.C. Sexton, F. Franchetti, S. Kral, C.W. Ueberhuber, and J. Lorenz, Large-scale electronic structure calculations of high-Z metals on the BlueGene/L platform, in Proceedings of the 2006 ACM/IEEE conference on Supercomputing. 2006, ACM: Tampa, Florida. p. 45.

313. Bylaska, E.J., K. Tsemekhman, S.B. Baden, J.H. Weare, and H. Jonsson, "Parallel Implementation of Gamma-Point Pseudopotential Plane-Wave DFT with Exact Exchange," Journal of Computational Chemistry, 2011, 32(1), 54-69.

314. Bylaska, E.J., K. Glass, D. Baxter, S.B. Baden, and J.H. Weare, "Hard scaling challenges for ab initio molecular dynamics capabilities in NWChem: Using 100,000 CPUs per second," Scidac 2009: Scientific Discovery through Advanced Computing, 2009, 180.

315. Bylaska, E.J., M. Holst, and J.H. Weare, "Adaptive Finite Element Method for Solving the Exact Kohn-Sham Equation of Density Functional Theory," Journal of Chemical Theory and Computation, 2009, 5(4), 937-948. 
316. Enkovaara, J., C. Rostgaard, J.J. Mortensen, J. Chen, M. Dulak, L. Ferrighi, J. Gavnholt, C. Glinsvad, V. Haikola, H.A. Hansen, H.H. Kristoffersen, M. Kuisma, A.H. Larsen, L. Lehtovaara, M. Ljungberg, O. Lopez-Acevedo, P.G. Moses, J. Ojanen, T. Olsen, V. Petzold, N.A. Romero, J. Stausholm-Moller, M. Strange, G.A. Tritsaris, M. Vanin, M. Walter, B. Hammer, H. Hakkinen, G.K.H. Madsen, R.M. Nieminen, J. Norskov, M. Puska, T.T. Rantala, J. Schiotz, K.S. Thygesen, and K.W. Jacobsen, "Electronic structure calculations with GPAW: a real-space implementation of the projector augmented-wave method," Journal of Physics-Condensed Matter, 2010, 22(25).

317. Fattebert, J.L. and F. Gygi, "Linear-scaling first-principles molecular dynamics with plane-waves accuracy," Physical Review B, 2006, 73(11).

318. Pask, J.E. and P.A. Sterne, "Finite element methods in ab initio electronic structure calculations," Modelling and Simulation in Materials Science and Engineering, 2005, 13(3), R71-R96.

319. Harrison, R.J., G.I. Fann, T. Yanai, and G. Beylkin, "Multiresolution quantum chemistry in multiwavelet bases," Computational Science - Iccs 2003, Pt Iv, Proceedings, 2003, 2660, 103 110.

320. Goedecker, S. and O.V. Ivanov, "Linear scaling solution of the Coulomb problem using wavelets," Solid State Communications, 1998, 105(11), 665-669.

321. Bowler, D.R., J.L. Fattebert, M.J. Gillan, P.D. Haynes, and C.K. Skylaris, "Introductory remarks: Linear scaling methods - Preface," Journal of Physics-Condensed Matter, 2008, 20(29).

322. Wang, Y.A., N. Govind, and E.A. Carter, "Orbital-free kinetic-energy density functionals with a density-dependent kernel," Physical Review B, 1999, 60(24), 16350-16358.

323. Smargiassi, E. and P.A. Madden, "Orbital-Free Kinetic-Energy Functionals for 1st-Principles Molecular-Dynamics," Physical Review B, 1994, 49(8), 5220-5226.

324. Ligneres, V.L.E., Advances in orbital-free density-functional theory. 2008.

325. Herring, C., "Explicit Estimation of Ground-State Kinetic Energies from Electron-Densities," Physical Review A, 1986, 34(4), 2614-2631.

326. Chai, J.D., V.L. Ligneres, G. Ho, E.A. Carter, and J.D. Weeks, "Orbital-free density functional theory: Linear scaling methods for kinetic potentials, and applications to solid $\mathrm{Al}$ and $\mathrm{Si}$," Chemical Physics Letters, 2009, 473(4-6), 263-267.

327. Berding, M.A., "Annealing conditions for intrinsic CdTe," Applied Physics Letters, 1999, 74(4), 552-554

328. Berding, M.A., A. Sher, and M. Vanschilfgaarde, "Defect Modeling Studies in Hgcdte and Cdte," Journal of Electronic Materials, 1995, 24(9), 1127-1135.

329. Carvalho, A., S. Oberg, and P.R. Briddon, "Intrinsic defect complexes in CdTe and ZnTe," Thin Solid Films, 2011, 519(21), 7468-7471.

330. Chang, Y.C., R.B. James, and J.W. Davenport, "Symmetrized-basis LASTO calculations of defects in CdTe and ZnTe," Physical Review B, 2006, 73(3).

331. Ko, E., M.M.G. Alemany, and J.R. Chelikowsky, "Viscosities of liquid CdTe near melting point from ab initio molecular-dynamics calculations," Journal of Chemical Physics, 2004, 121(2), 942945.

332. Ko, E., M.M.G. Alemany, J.J. Derby, and J.R. Chelikowsky, "Ab initio simulations of nonstoichiometric CdxTe1-x liquids," Journal of Chemical Physics, 2005, 123(8).

333. Lordi, V., "Mechanisms and Kinetics of Tellurium Precipitation in CdTe-based Materials. ," Bulletin of the American Physical Society, 2012, 57.

334. Aberg, D., P. Erhart, and V. Lordi, Contributions of point defects, chemical disorder, and thermal vibrations to electronic properties of Cd1-xZnxTe alloys, in arXiv preprint arXiv:1210.3305. 2012.

335. Wei, S.H. and S.B. Zhang, "First-principles study of doping limits of CdTe," Physica Status Solidi B-Basic Research, 2002, 229(1), 305-310.

336. Lany, S., H. Wolf, and T. Wichert, "Density functional theory calculations establish the experimental evidence of the DX center atomic structure in CdTe," Physical Review Letters, 2004, $92(22)$. 
337. Lany, S., V. Ostheimer, H. Wolf, and T. Wichert, "Vacancies in CdTe: experiment and theory," Physica B-Condensed Matter, 2001, 308, 958-962.

338. Lany, S., V. Ostheimer, H. Wolf, and T. Wichert, "Calculated electric field gradients and electronic properties of acceptors in CdTe," Hyperfine Interactions, 2001, 136, 619-625.

339. Hamann, J., L. Worschech, S. Lany, W. Ossau, V. Ostheimer, C. Schmitz, H. Wolf, and T. Wichert, "Defect-induced bound-exciton lines in hydrogen-doped CdTe: Zeeman spectroscopy," Proceedings of the 25th International Conference on the Physics of Semiconductors, Pts I and Ii, 2001, 87, 1439-1440.

340. Bhattacharya, S.K. and A. Kshirsagar, "Ab initio calculations of structural and electronic properties of CdTe clusters," Physical Review B, 2007, 75(3).

341. Yao, K.L., G.Y. Gao, Z.L. Liu, L. Zhu, and Y.L. Li, "Half-metallic ferromagnetic semiconductors of V-and Cr-doped CdTe studied from first-principles pseudopotential calculations," Physica BCondensed Matter, 2005, 366(1-4), 62-66.

342. Du, M.H., H. Takenaka, and D.J. Singh, "Carrier compensation in semi-insulating CdTe: Firstprinciples calculations," Physical Review B, 2008, 77(9).

343. Rak, Z.S., S.D. Mahanti, and K.C. Mandal, "Ab Initio Studies of Hydrogen Defects in CdTe," Journal of Electronic Materials, 2009, 38(8), 1539-47.

344. Miotto, R., F.D. Kiss, and A.C. Ferraz, "Oxygen adsorption on CdTe(110)," Surface Science, 2003, 525(1-3), 24-32.

345. Bylaska, E.J., K. Tsemekhman, and F. Gao, "New development of self-interaction corrected DFT for extended systems applied to the calculation of native defects in 3C-SiC," Physica Scripta, 2006, T124, 86-90.

346. Apra, E., E.J. Bylaska, D.J. Dean, A. Fortunelli, F. Gao, P.S. Krstic, J.C. Wells, and T.L. Windus, "NWChem for materials science," Computational Materials Science, 2003, 28(2), 209-221.

347. Kluner, T., N. Govind, Y.A. Wang, and E.A. Carter, "Periodic density functional embedding theory for complete active space self-consistent field and configuration interaction calculations: Ground and excited states," Journal of Chemical Physics, 2002, 116(1), 42-54.

348. Govind, N., Y.A. Wang, A.J.R. da Silva, and E.A. Carter, "Accurate ab initio energetics of extended systems via explicit correlation embedded in a density functional environment," Chemical Physics Letters, 1998, 295(1-2), 129-134.

349. Govind, N., Y.A. Wang, and E.A. Carter, "Electronic-structure calculations by first-principles density-based embedding of explicitly correlated systems," Journal of Chemical Physics, 1999, 110(16), 7677-7688.

350. Zhu, T.H., W. Pan, and W.T. Yang, "Structure of solid-state systems from embedded-cluster calculations: A divide-and-conquer approach," Physical Review B, 1996, 53(19), 12713-12724.

351. Lento, J., J.L. Mozos, and R.M. Nieminen, "Charged point defects in semiconductors and the supercell approximation," Journal of Physics-Condensed Matter, 2002, 14(10), 2637-2645.

352. Leslie, M. and M.J. Gillan, "The Energy and Elastic Dipole Tensor of Defects in Ionic-Crystals Calculated by the Supercell Method," Journal of Physics C-Solid State Physics, 1985, 18(5), 973 982.

353. Makov, G. and M.C. Payne, "Periodic Boundary-Conditions in Ab-Initio Calculations," Physical Review B, 1995, 51(7), 4014-4022.

354. Schultz, P.A., "Charged local defects in extended systems," Physical Review Letters, 2000, 84(9), 1942-1945.

355. E, W.N., W.Q. Ren, and E. Vanden-Eijnden, "Simplified and improved string method for computing the minimum energy paths in barrier-crossing events," Journal of Chemical Physics, 2007, 126(16).

356. Jonsson, H., G. Mills, and K.W. Jacobsen, "Nudged elastic band method for finding minimum energy paths," Classical and quantum dynamics in condensed phase simulation: proceeding of the the International School of Physics, 1998, 385. 
357. Barducci, A., M. Bonomi, and M. Parrinello, "Metadynamics," Wiley Interdisciplinary ReviewsComputational Molecular Science, 2011, 1(5), 826-843.

358. Quigley, D. and P.M. Rodger, "A metadynamics based approach to sampling crystallization events," Molecular Simulation, 2009, 35, 613-623.

359. Peet, M., S.S. Babu, M.K. Miller, and H.K.D.H. Bhadeshia, "Three-dimensional atom probe analysis of carbon distribution in low-temperature bainite," Scripta Materialia, 2004, 50(10), $1277-1281$.

360. Rusing, J., D. Isheim, O.C. Hellman, J.A. Vandenbroucke, and D.N. Seidman, "Analysis of threedimensional atom-probe data by the proximity histogram," Microscopy and Microanalysis, 2000, 6, 437-444.

361. Ohkub, T., K. Hono, J.C. Oh, and T. Muka, "TEM and 3D AP characterization of an agehardened Mg-Ca-Zn alloy," Scripta Materialia, 2005, 53, 675-679.

362. Uchic, M.D., M.D. Graef, R. Wheeler, and D.M. Dimiduk, "Microstructural tomography of a superalloy using focused ion beam microscopy," Ultramicroscopy, 2009, 109(10), 1229-1235.

363. Dunn, D.N. and R. Hull, "Reconstruction of three-dimensional chemistry and geometry using focused ion beam microscopy," App. Phys. Lett., 1999, 75, 3414-3416.

364. Alkemper, J. and P.W. Voorhees, "Quantitative serial sectioning analysis," J. Microscopy, 2001, 201, 388-394.

365. Mullens, H.M., J.E. Spowart, and B.T. Puchala, "Collecting and analyzing microstructures in three dimensions: a fully automated approach," J. of Metals, 2003, 55, 35-37.

366. Lauridsen, E.M., S. Schmidt, R.M. Suter, U. Lienert, L. Margulies, T. Lorentzen, H.F. Poulsen, S.F. Nielsen, and D.J. Jensen, "Three-dimensional maps of grain boundaries and the stress state of individual grains in polycrystals and powders," J. Appl. Cryst., 2001, 34, 751-756.

367. Ganapathysubramanian, B. and N. Zabaras, "A stochastic multiscale framework for modeling flow through random heterogeneous porous media," J. Comput. Phys., 2009, 228(2), 591--618.

368. Ganapathysubramanian, B. and N. Zabaras, "A non-linear dimension reduction methodology for generating data-driven stochastic input models," J. Comput. Phys., 2008, 227(13), 6612--6637.

369. Tenenbaum, J.B., V.d. Silva, and J.C. Langford, "A Global Geometric Framework for Nonlinear Dimensionality Reduction," Science, 2000, 290(5500), 2319-2323.

370. Roweis, S.T. and L.K. Saul, "Nonlinear dimensionality reduction by locally linear embedding," Science, 2000, 290, 2323--2326.

371. Ozakin, A. and A.G. Gray, Submanifold Density Estimation, in Advances in Neural Information Processing Systems, M. Press, Editor. 2010, MIT Press.

372. Ozakin, A., N. Vasiloglou, and A.G. Gray, Density-Preserving Maps, in Density-Preserving Maps. In Manifold Learning Theory and Applications. 2012, Taylor and Francis Group.

373. Tino, P. and I. Nabney, "Hierarchical GTM: constructing localized nonlinear projection manifolds in a principled way," IEEE Transactions on Pattern Analysis and Machine Intelligence, 2002, 24(5), 639-56.

374. Wang, X., P. Tino, and M. Fardal, Multiple manifolds learning framework based on hierarchical mixture density model, in Machine Learning and Knowledge Discovery in Databases, W. Daelemans, B. Goethals, and K. Morik, Editors. 2008, Springer Berlin / Heidelberg, 2008. p. 566-581.

375. Wen, B. and N. Zabaras, "Data-driven multiscale uncertainty quantification based on biorthogonal decomposition," Comp. Materials Science, 2012, Submitted.

376. Mortensen, J.J., K. Kaasbjerg, S.L. Frederiksen, J.K. Nrskov, J.P. Sethna, and K.W. Jacobsen, "Bayesian error estimation in density-functional theory," Physical Review Letters, 2005, 95(21).

377. Frederiksen, S.L., K.W. Jacobsen, K.S. Brown, and J.P. Sethna, "Bayesian ensemble approach to error estimation of interatomic potentials," Physical Review Letters, 2004, 93(16), 165501-1.

378. Curtin, W.A., D.L. Olmsted, and J.L.G. Hector, "A predictive mechanism for dynamic strain ageing in aluminium-magnesium alloys," Nat. Mater., 2006, 5, 875-880. 
379. Yasi, J.A., J.L.G. Hector, and D.R. Trinkle, "Prediction of thermal cross-slip stress in magnesium alloys from direct first principles data," Acta Mater., 2011, 59, 5652-5660.

380. Ma, X. and N. Zabaras, "An adaptive hierarchical sparse grid collocation algorithm for the solution of stochastic differential equations," Journal of Computational Physics, 2009, 228(8), 3084-113.

381. Ma, X. and N. Zabaras, "An adaptive high-dimensional stochastic model representation technique for the solution of stochastic partial differential equations," Journal of Computational Physics, 2010, 229(10), 3884-915.

382. Bilionis, I. and N. Zabaras, "Multidimensional Adaptive Relevance Vector Machines: Applications to Uncertainty Quantification," SIAM J. Scientific Computing, 2011, Submitted.

383. Bilionis, I. and N. Zabaras, "Multi-output Local Gaussian Process Regression: Applications to Uncertainty Quantification," Journal of Computational Physics, 2011, Submitted.

384. Tewary, V.K. and R. Thomson, "Lattice statics of interfaces and interfacial cracks in bimaterial solids," J. Mater. Res., 1992, 7(4), 1018-1028.

385. Ohsawa, K., E. Kuramoto, and T. Suzuki, "Lattice statics Green's function for a semi-infinite crystal," Philos. Mag. A, 1996, 74(2), 431-449.

386. Masuda-Jindo, K. and V.V. Hung, "Atomistic Study of Fracture of Crystalline Materials by Lattice Green's Function Method: Effects of Anharmonicity of Lattice Vibration," J. Phys. Soc. Japan, 2004, 73(5), 1205-1215.

387. Tschöp, W., K. Kremer, J. Batoulis, T. Berger, and O. Hahn, "Simulation of polymer melts. I. Coarse-graining procedure for polycarbonates," Acta Polym., 1998, 49(2-3), 61--74.

388. Reith, D., M. Putz, and F. Muller-Plathe, "Deriving effective mesoscale potentials from atomistic simulations," J. Comput. Chem., 2003, 24(13), 1624--1636.

389. Soper, A.K., "Empirical potential Monte Carlo simulation of fluid structure," Chem. Phys., 1996, 202(2, Äi3), 295-306.

390. Lyubartsev, A.P. and A. Laaksonen, "Calculation of effective interaction potentials from radial distribution functions: A reverse Monte Carlo approach," Phys. Rev. E, 1995, 52, 3730--3737.

391. Noid, W.G., J. Chu, G.S. Ayton, and G.A. Voth, "Multiscale Coarse-Graining and Structural Correlations: Connections to Liquid-State Theory," Journal Phys. Chem. B, 2007, 111(16), 41164127.

392. Izvekov, S. and G.A. Voth, "Multiscale coarse graining of liquid-state systems," J. Chem. Phys., 2005, 123(13), 134105.

393. Shell, M.S., "The relative entropy is fundamental to multiscale and inverse thermodynamic problems," J. Chem. Phys., 2008, 129(14), 144108.

394. Carmichael, S.P. and M.S. Shell, "A New Multiscale Algorithm and Its Application to CoarseGrained Peptide Models for Self-Assembly," J. Phys. Chem. B, 2012, 116(29), 8383-8393.

395. Pritchard-Bell, A. and M.S. Shell, "Smoothing Protein Energy Landscapes by Integrating Folding Models with Structure Prediction," Biophys. J., 2011, 101, 2251-2259.

396. Chaimovich, A. and M.S. Shell, "Coarse-graining errors and numerical optimization using a relative entropy framework," J. Chem. Phys., 2011, 134(9), 094112.

397. Chaimovich, A. and M.S. Shell, "Anomalous waterlike behavior in spherically-symmetric water models optimized with the relative entropy," Phys. Chem. Chem. Phys., 2009, 11, 1901-1915.

398. Asthalter, T. and M. Walter, "Visualizing covalency and multiple bonds in terms of the electronic single-particle density matrix," Zeitschrift fur Physikalische Chemie, 2001, 215(10), 1277.

399. Schmider, H., "Wigner functions and bond orders," Zeitschrift fur Physikalische Chemie, 2006, 220(7), 859-884.

400. $\mathrm{Yu}, \mathrm{M}$. and D.R. Trinkle, "Au/ $\mathrm{TiO}_{2}(110)$ interfacial reconstruction stability from ab initio," J. Phys. Chem. C, 2011, 115, 17799-17805.

401. Ramprasad, R., "First-principles energy and stress fields in defected materials," J. Phys.: Condens. Matter, 2002, 14, 5497-5516. 
402. Chetty, N. and R.M. Martin, "First-principles energy density and its applications to selected polar surfaces," Phys. Rev. B, 1992, 45, 6074-6088.

403. Sundararaghavan, V. and N. Zabaras, "A statistical learning approach for the design of polycrystalline materials," Stat. Anal. Data Min., 2009, 1, 306-321.

404. Bishop, C.M., M. Svensen, and C.K.I. Williams, "GTM: the generative topographic mapping," Neural Computation, 1998, 10(1), 215-34.

405. Hu, S.Y. and C.H. Henager Jr, "Phase-field simulation of void migration in a temperature gradient," Acta Materialia, 2010, 58, 3230-3237.

406. Millet, P.C. and M. Tonks, "Application of phase-field modeling to irradiation effects in materials," Current Opinions in Solid State and Material Science, 2011, 15, 125-133.

407. Wang, L., J. Lee, M. Anitescu, A.E. Azab, L.C. McInnes, T. Munson, and B. Smith, A differential variational inequality approach for the simulation of heterogeneous materials, in SciDac 2011 Conference. 2011: Denver, Colorado.

408. Gomez, H. and T.J.R. Hughes, "Provably unconditionally stable, second-order time-accurate, mixed variational methods for phase-field models," preprint, 2011.

409. Dorr, M.R., J.L. Fattebert, M.E. Wickett, J.F. Belak, and P.E.A. Turchi, "A numerical algorithm for the solution of a phase-field model of polycrystalline materials," Journal of Computational Physics, 2010, 229(Copyright 2010, The Institution of Engineering and Technology), 626-41.

410. Gander, M.J. and S. Vandewalle, "Analysis of the parareal time-parallel time-integration method," SIAM Journal on Scientific Computing, 2007, 29(Copyright 2008, The Institution of Engineering and Technology), 556-78.

411. Maday, Y. and G. Turinici, "A parareal in time procedure for the control of partial differential equations," Comptes Rendus de l'Academie des Sciences, Serie I (Mathematique), 2002, 335(Copyright 2002, IEE), 387-92.

412. Bal, G. and Y. Maday, "A parareal time discretization for non-linear PDE's with application to pricing of an American put," Lecture Notes Comput.. Eng., 2002, 23.

413. Garrido, I., B. Lee, G.E. Fladmark, and M.S. Espedal, "Convergent iterative schemes for time parallelization," Mathematics of Computation, 2006, 75(Copyright 2006, The Institution of Engineering and Technology), 1403-28.

414. Donev, A., V.V. Bulatov, T. Oppelstrup, G.H. Gilmer, B. Sadigh, and M.H. Kalos, "A FirstPassage Kinetic Monte Carlo algorithm for complex diffusion-reaction systems," Journal of Computational Physics, 2010, 229, 3214-3236.

415. Hu, S. and C.H. Henager, Jr., "Phase-field modeling of void lattice formation under irradiation," Journal of Nuclear Materials, 2009, 394, 155-9.

416. Kerisit, S., K.M. Rosso, and B.D. Cannon, "Kinetic Monte Carlo model of scintillation mechanisms in CsI and CsI(Tl)," IEEE Transactions on Nuclear Science, 2008, 55(3), 1251-1258.

417. Gao, F., L.W. Campbell, Y. Xie, R. Devanathan, A.J. Peurrung, and W.J. Weber, "Electron-hole pairs created by photons and intrinsic properties in detector materials," IEEE Transactions on Nuclear Science, 2008, 55(3), 1079-1085.

418. Gao, F., L.W. Campbell, R. Devanathan, Y.L. Xie, Y. Zhang, A.J. Peurrung, and W.J. Weber, "Gamma-ray interaction in Ge: A Monte Carlo simulation," Nuclear Instruments and Methods in Physics Research, Section B: Beam Interactions with Materials and Atoms, 2007, 255(1 SPEC ISS), 286-290.

419. Gao, F., L.W. Campbell, R. Devanathan, Y. Xie, L.R. Corrales, A.J. Peurrung, and W.J. Weber, "Monte Carlo method for simulating \&gamma;-ray interaction with materials: A case study on $\mathrm{Si}$," Nuclear Instruments and Methods in Physics Research, Section A: Accelerators, Spectrometers, Detectors and Associated Equipment, 2007, 579(1), 292-296.

420. Alexander, H., "On dislocation generation in semiconductor crystals," Radiation Effects and Defects in Solids, 1989, 111-112(1-2), 1-12.

421. Peissker, E., P. Haasen, and H. Alexander, "Anisotropic plastic deformation of indium antimonide," Philosophical Magazine, 1962, 7(80), 1279-1303. 